\title{
A Tutorial on
}

Synthetic Aperture Radar

\section{ALBERTO MOREIRA, PAU PRATS-IRAOLA, MARWAN YOUNIS, GERHARD KRIEGER, IRENA HAJNSEK, AND KONSTANTINOS P. PAPATHANASSIOU}

Microwaves and Radar Institute of the German Aerospace Center (DLR), Germany

Abstract-Synthetic Aperture Radar (SAR) has been widely used for Earth remote sensing for more than 30 years. It provides high-resolution, day-and-night and weather-independent images for a multitude of applications ranging from geoscience and climate change research, environmental and Earth system monitoring, 2-D and 3-D mapping, change detection, 4-D mapping (space and time), security-related applications up to planetary exploration. With the advances in radar technology and geo/bio-physical parameter inversion modeling in the 90s, using data from several airborne and spaceborne systems, a paradigm shift occurred from the development driven by the technology push to the user demand pull. Today, more than 15 spaceborne SAR systems are being operated for innumerous applications. This paper provides first a tutorial about the SAR principles and theory, followed by an overview of

Digital Object Identifier 10.1109/MGRS.2013.224830 Date of publication: 17 April 2013 established techniques like polarimetry, interferometry and differential interferometry as well as of emerging techniques (e.g., polarimetric SAR interferometry, tomography and holographic tomography). Several application examples including the associated parameter inversion modeling are provided for each case. The paper also describes innovative technologies and concepts like digital beamforming, Multiple-Input Multiple-Output (MIMO) and bi- and multi-static configurations which are suitable means to fulfill the increasing user requirements. The paper concludes with a vision for SAR remote sensing.

\section{INTRODUCTION}

Cynthetic Aperture Radar (SAR) has entered into a golden age. More than 15 spaceborne SAR sensors are being operated today and 10 new SAR systems will be launched within the next 5 years. SAR is unique in its imaging capability: It provides high-resolution twodimensional images independent from daylight, cloud 
TABLE 1. COMMONLY USED FREQUENCY BANDS FOR SAR SYSTEMS AND THE CORRESPONDING FREQUENCY AND WAVELENGTH RANGES. APPLICATION EXAMPLES ARE: 1) FOLIAGE PENETRATION, SUBSURFACE IMAGING AND BIOMASS ESTIMATION IN P- AND L-BAND; 2) AGRICULTURE, OCEAN, ICE OR SUBSIDENCE MONITORING IN L-, C-, S- AND X-BAND; 3) SNOW MONITORING IN X- AND KU-BAND; AND 4) VERY HIGH-RESOLUTION IMAGING IN X-AND KA-BAND. MOST USED FREQUENCY BANDS ARE L-, C- AND X-BAND.

$\begin{array}{llllllll}\text { Frequency Band } & \text { Ka } & \text { Ku } & \mathbf{X} & \mathbf{C} & \mathbf{S} & \mathbf{L} & \mathbf{P} \\ \text { Frequency [GHz] } & 40-25 & 17.6-12 & 12-7.5 & 7.5-3.75 & 3.75-2 & 2-1 & 0.5-0.25 \\ \text { Wavelength [cm] } & 0.75-1.2 & 1.7-2.5 & 2.5-4 & 4-8 & 8-15 & 15-30 & 60-120\end{array}$

coverage and weather conditions [1]-[9]. It is predestined to monitor dynamic processes on the Earth surface in a reliable, continuous and global way. SAR systems have a side-looking imaging geometry and are based on a pulsed radar installed on a platform with a forward movement. The radar system transmits electromagnetic pulses with high power and receives the echoes of the backscattered signal in a sequential way. Typical values for the pulse repetition frequency range from a few hundred to a few thousand Hertz for airborne and spaceborne systems, respectively. The swath width varies typically from a few kilometers to $20 \mathrm{~km}$ in the airborne case and from 30 to $500 \mathrm{~km}$ in the spaceborne case. The transmitted pulse interacts with the Earth surface and only a portion of it is backscattered to the receiving antenna which can be the same as the transmit antenna (for a monostatic radar) or a different one (for a bi- or multi-static radar). The amplitude and phase of the backscattered signal depends on the physical (i.e., geometry, roughness) and electrical properties (i.e., permittivity) of the imaged object. Depending on the frequency band, considerable penetration can occur so that the imaged objects and media must be modeled as a volume (e.g., vegetation, ice and snow, dry soil). More penetration of the electromagnetic pulses in media will occur for radar systems using longer wavelengths which usually have an accentuated volume contribution in the backscattered signal. Commonly used frequency bands in SAR systems and the associated wavelength ranges are shown in Table 1.

The simplest radar system provides a 2-D reflectivity map of the imaged area, i.e., targets with high backscattered signal are identified as bright spots in the radar images and flat smooth surfaces as dark areas. The flight direction is denoted as azimuth and the line-of-sight as slant range direction. Until the 50s imaging radars were denoted as SLAR (side-looking airborne radar) and did not use the principle of the synthetic aperture. This led to a moderate azimuth resolution which deteriorates as the range increases. For example, an X-band SLAR system with a 3-meter antenna $d_{a}$ has an azimuth antenna beamwidth of

$$
\Theta_{a}=\frac{\lambda}{d_{a}}=\frac{0.03 \mathrm{~m}}{3 \mathrm{~m}}=0.01 \mathrm{rad}
$$

where $\lambda$ is the wavelength. The azimuth resolution $\delta_{a}$ is given by the smallest separation between two point targets that can be detected by the radar. In the SLAR case this is given by the illumination length of the azimuth antenna beam on the ground. Assuming a range distance, $r_{0}$, from the antenna to the targets of $5 \mathrm{~km}$ yields

$$
\delta_{a}=\frac{\lambda}{d_{a}} \cdot r_{0}=\Theta_{a} \cdot r_{0}=0.01 \cdot 5000 \mathrm{~m}=50 \mathrm{~m} .
$$

The moderate to low azimuth resolution has been the main drawback of the SLAR system. With an invention by Carl Wiley in 1951 followed by a patent application in 1954 [10], this limitation has been overcome by the use of a coherent radar and the principle of Doppler beam sharpening [11], leading to an improvement of the azimuth resolution. In the following years, this concept was extended to the principle of the synthetic aperture as it is known today [12]. The resulting azimuth resolution becomes equal to half the azimuth antenna length $\left(\delta_{a}=d_{a} / 2\right)$ and is independent of the range distance. This means that the azimuth resolution in the previous example is equal to $1.5 \mathrm{~m}$, i.e., more than 30 times better than the resolution of the real aperture SLAR system. For a spaceborne SAR system with equal azimuth antenna length, the azimuth resolution will be the same (i.e., $1.5 \mathrm{~m}$ ). The invention of the SAR principle represented a major milestone for the development of airborne and spaceborne imaging radar systems in the decades to follow.

The discovery and initial developments of SAR systems in the 50s and 60s were dominated by military research and use for reconnaissance purposes and man-made target detection. SAR images represent however much more than just a 2-D reflectivity map. In the 70s and 80 s several airborne systems were developed for civilian applications with the ultimate goal to retrieve geo/bio-physical parameters from the Earth surface. From the beginning, airborne SAR systems were always a step ahead in the technological development, allowing the demonstration of new techniques and applications that were later implemented in spaceborne SAR missions.

The launch of Seasat in 1978, the first civilian SAR satellite, as well as the advancement in digital technology boosted the SAR development. We can summarize this time 
as the discovery time with a strong believe on the potential and future of radar remote sensing. The launch of the ERS1/2 (C-band), JERS-1 (L-band) and Radarsat-1 (C-band) satellites in the 90s represented further milestones in the spaceborne SAR development in Europe, Japan and Canada, respectively. SAR techniques like polarimetry for improved parameter retrieval, interferometry for deriving the surface topography and differential interferometry for the measurement of Earth surface displacements were developed in the 80s and 90s [13]-[16]. The application fields of these techniques were catapulted by the shuttle missions SIRC/X-SAR (Shuttle Imaging Radar mission with X-, C- and L-band radars, the latter two being fully polarimetric) in 1994 and the Shuttle Radar Topography Mission (SRTM) at X-band and C-band in 2000. A further milestone in the SAR development was associated to differential SAR interferometry with permanent scatterers (PS) for subsidence monitoring [17], a technique that was developed using data from

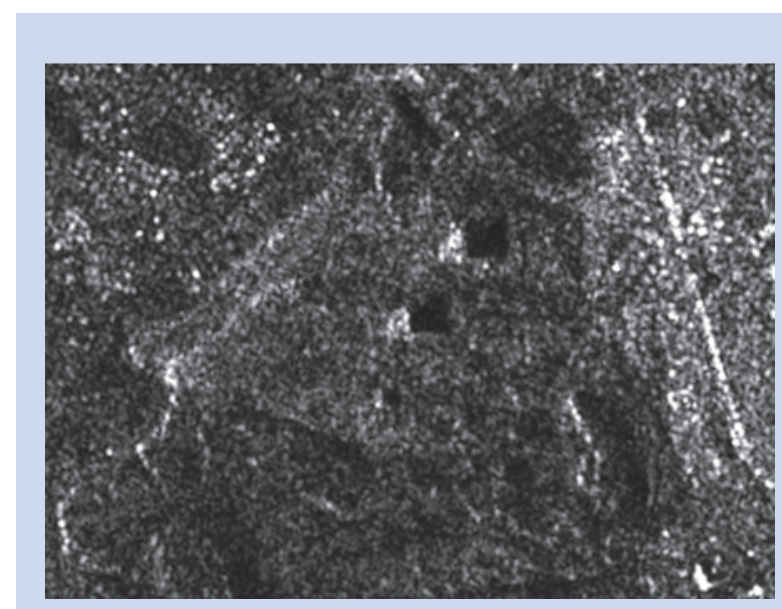

(a)

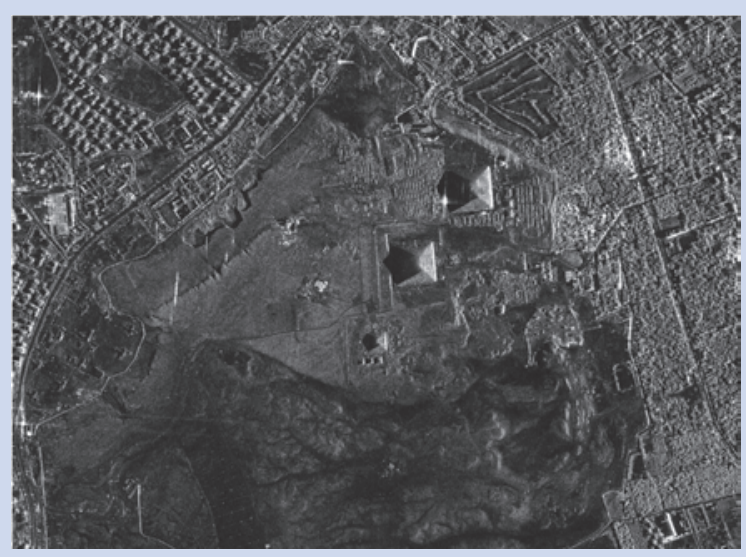

(b)

FIGURE 1. Comparison of a SAR image corresponding to the state of the art during the $90 \mathrm{~s}$ [(a) ca. 20 m resolution, C-band, radar illumination from the left] and the current generation of SAR satellites available since 2007 [(b) $1 \mathrm{~m}$ resolution, $\mathrm{X}$-band, radar illumination from the right]. The images show the pyramids of Giza, Egypt.
ERS-1/2 and later ENVISAT/ASAR (C-band). The latter was the first SAR satellite using the antenna technology with transmit/receive modules for achieving greater flexibility in the steering of the radar antenna beam and therefore in the selection of different imaging modes. In the last 10 years, considerable progress has been achieved with polarimetric SAR interferometry (Pol-InSAR) [18] and tomography for obtaining information of volume scatterers [19]. Tomography has been also used in combination with PS techniques to solve the problem associated with the layover effect in urban areas. Most recently, holographic tomography has been proposed for generating a $360^{\circ}$ imaging view of volume scatterers [20].

With the launch of the bi-static SAR satellites TerraSAR-X and TanDEM-X (X-band), the COSMO-SkyMed satellite constellation (X-band) as well as Radarsat-2 (C-band) a new class of SAR satellites was introduced providing images with resolution in the meter regime. Fig. 1 shows a comparison of a SAR image with moderate resolution corresponding to the state of the art in the 90s and a SAR image obtained with the new generation of high-resolution SAR satellites.

The trend for future systems shows the need for an increased information content in SAR images that can be achieved by multi-channel operation (polarimetry, multifrequency), improved range and azimuth resolution, time series (frequent revisit of the same area) as well as observation angle diversity (interferometry and tomography). These user requirements push the development of new technologies (e.g., digital beamforming, MIMO, bi- and multi-static, large reflector antennas) that are shaping the future of spaceborne SAR systems with the ultimate goal to allow a wide-swath high-resolution monitoring of dynamic processes on the Earth surface in a quasi-continuous way. Table 2 provides an overview of spaceborne SAR sensors (a recent compilation of airborne SAR sensors is given in [21]). More than 10 SAR satellites will be launched within the next 5 years. A golden age for SAR remote sensing has started!

This paper is organized as follows. Section II provides an introduction to the SAR principles, image formation process and SAR image properties. Sections III to VI explain the techniques of polarimetry, interferometry, differential interferometry, polarimetric SAR interferometry and tomography along with application examples. Section VII provides an overview on emerging technologies for future spaceborne SAR systems driven by the user requirements. Section VIII concludes the paper and provides a vision for SAR remote sensing.

\section{BASIC SAR PRINCIPLES}

A Synthetic Aperture Radar is an imaging radar mounted on a moving platform. Similar to a conventional radar, electromagnetic waves are sequentially transmitted and the backscattered echoes are collected by the radar antenna. In the case of SAR the consecutive time of transmission/reception translates into different positions due to the platform movement. An appropriate coherent 
TABLE 2. OVERVIEW OF SPACEBORNE SAR SENSORS AND THEIR MAIN CHARACTERISTICS [22].

\begin{tabular}{|c|c|c|}
\hline SENSOR & OPERATION & $\begin{array}{l}\text { FREQUENCY BAN } \\
\text { (POLARIZATION) }\end{array}$ \\
\hline Seasat & 1978 & $\mathrm{~L}(\mathrm{HH})$ \\
\hline ERS- $1 / 2$ & $\begin{array}{l}1991-2000 / \\
1995-2011\end{array}$ & $\mathrm{C}(\mathrm{VV})$ \\
\hline J-ERS-1 & 1992-1998 & $\mathrm{L}(\mathrm{HH})$ \\
\hline SIR-C/ X-SAR & April and October 1994 & $\begin{array}{l}\text { L \& C (quad) } \\
\text { X (VV) }\end{array}$ \\
\hline Radarsat-1 & 1995-today & $\mathrm{C}(\mathrm{HH})$ \\
\hline SRTM & Feb. 2000 & $\begin{array}{l}\mathrm{C}(\mathrm{HH}+\mathrm{VV}) \text { and } \\
\mathrm{X}(\mathrm{VV})\end{array}$ \\
\hline ENVISAT/ ASAR & $2002-2012$ & C (dual) \\
\hline ALOS/PaISAR & $2006-2011$ & $L$ (quad) \\
\hline $\begin{array}{l}\text { TerraSAR-X/ } \\
\text { TanDEM-X }\end{array}$ & $\begin{array}{l}\text { 2007-today } \\
\text { 2010-today }\end{array}$ & $X$ (quad) \\
\hline Radarsat-2 & 2007-today & C (quad) \\
\hline Cosmo-SkyMed-1/4 & 2007 ... 2010-today & X (dual) \\
\hline RISAT-1 & 2012-today & C (quad) \\
\hline $\mathrm{HJ}-1 \mathrm{C}$ & 2012-today & $\mathrm{S}(\mathrm{VV})$ \\
\hline Kompsat-5 & Launch scheduled in 2013 & X (dual) \\
\hline PAZ & Launch scheduled in 2013 & $X$ (quad) \\
\hline ALOS-2 & Launch scheduled in 2013 & L (quad) \\
\hline Sentinel-1a/1b & $\begin{array}{l}\text { Launch scheduled in } \\
2013 / 2015\end{array}$ & C (dual) \\
\hline $\begin{array}{l}\text { Radarsat Constella- } \\
\text { tion- } 1 / 2 / 3\end{array}$ & Launch scheduled in 2017 & $C$ (quad) \\
\hline SAOCOM-1/2 & $\begin{array}{l}\text { Launch scheduled in } \\
2014 / 2015\end{array}$ & $L$ (quad) \\
\hline
\end{tabular}

\section{COMMENTS}

First civilian SAR satellite, operation for only ca. three months

European Remote Sensing Satellites (first European SAR satellites)

Japanese Earth Resource Satellite

(first Japanese SAR satellite)

Shuttle imaging radar mission, first demonstration of spaceborne multi-frequency SAR

First Canadian SAR satellite, swath width of up to $500 \mathrm{~km}$ with ScanSAR imaging mode Shuttle Radar Topography Mission, first spaceborne interferometric SAR

First SAR satellite with Transmit/Receive module technology, swath width up to $400 \mathrm{~km}$

Advanced Land Observing Satellite (Daichi), swath width up to $360 \mathrm{~km}$

First bi-static radar in space, resolution up to $1 \mathrm{~m}$, global topography available by end of 2014

Resolution up to: $1 \mathrm{~m} \times 3 \mathrm{~m}$ (azimuth $\times$ range), swath width up to $500 \mathrm{~km}$

Constellation of four satellites, up to $1 \mathrm{~m}$ resolution

Follow-on satellite (RISAT-1a) to be launched in 2016, RISAT-3 (L-band) in development

Constellation of four satellites, first satellite launched in 2012

Korea Multi-Purpose Satellite 5, resolution up to $1 \mathrm{~m}$

Constellation with TerraSAR-X and TanDEM-X planned

Resolution up to: $1 \mathrm{~m} \times 3 \mathrm{~m}$ (azimuth $\times$ range), swath width up to $490 \mathrm{~km}$

Constellation of two satellites, swath width up to $400 \mathrm{~km}$

Constellation of three satellites, swath width up to $500 \mathrm{~km}$

Constellation of two satellites, fully polarimetric
INSTITUTION, COUNTRY NASA/JPL, USA

ESA, Europe

JAXA, Japan

NASA/JPL, USA

DLR, Germany

ASI, Italy

CSA, Canada

NASA/JPL, USA

DLR, Germany

ASI, Italy

ESA, Europe

JAXA, Japan

DLR/Astrium,

Germany

CSA, Canada

$\mathrm{ASI} / \mathrm{MiD}$, Italy

ISRO, India

CRESDA/CAST/ NRSCC, China

KARI, Korea

CDTI, Spain

JAXA, Japan

ESA, Europe

CSA, Canada

CONAE, Argentina combination of the received signals allows the construction of a virtual aperture that is much longer than the physical antenna length. This basic attribute of SAR is the origin of its name "synthetic aperture," giving it the property of being an imaging radar. In the case of SAR the radar image results from processing the raw data (i.e., after forming the synthetic aperture) and represents a measure of the scene reflectivity. The following section gives a brief overview of SAR, introducing the theory necessary for the following chapters. The intention here is to give an easy understanding of the subject and to avoid detailed descriptions and derivations for which interested readers are referred to a wealth of publications on SAR [1]-[9], [23]. In particular, the tutorial paper by Tomiyasu [4] gives a very comprehensive view about the SAR principle and image formation.

SAR sensors commonly utilize frequency modulated pulsed waveforms for transmission, the so-called chirp signals. The amplitude of the transmitted waveform is constant during the pulse time $\tau$, while the instantaneous frequency is varied in a linear manner over time $t$ according to $f_{i}=k_{r} \cdot t$ where $k_{r}$ is known as the chirp rate, yielding the bandwidth $B_{r}=k_{r} \tau$. This is followed by the echo window time during which the radar "listens" to the scattered echoes and stores 
the received signals on-board. When referring to the time in the range direction, it is often denoted to as fast time which is an allusion to the velocity of the electromagnetic waves travelling at the speed of light. The transmission and listen procedure is repeated every PRI seconds, where the pulse repetition interval (PRI) is the reciprocal of the pulse repetition frequency $P R I=1 / P R F$. Fig. 2 illustrates the typical SAR geometry, where the platform moves in the azimuth or along-track direction, whereas the slant range is the direction perpendicular to the radar's flight path. The swath width gives the ground-range extent of the radar scene, while its length depends on the data take duration, i.e., how long the radar is turned on.

At any time $t$, the distance between the radar moving at constant velocity $v$ and a point on the ground, described by its coordinates $(x, y, z)=\left(x_{0}, 0, \Delta h\right)$, is easily obtained applying Pythagoras' theorem

$$
r(t)=\sqrt{r_{0}^{2}+(v t)^{2}} \approx r_{0}+\frac{(v t)^{2}}{2 r_{0}} \text { for } v t / r_{0} \ll 1,
$$

where, without loss of generality $t=t_{0}=0$ is the time of closest approach, when the distance is minimum and $r\left(t_{0}\right)=r_{0}=\sqrt{(H-\Delta h)^{2}+x_{0}^{2}}$ with the platform height $H$. In general the distance $r_{0}$ is much larger than $v t$ during the illumination time $T_{i l l}$ a point on the ground is observed; this allows expanding $r(t)$ into a Taylor series and neglecting all but the first two terms, which yields the approximation on the right-hand side of (3). In the above expression the time, given through the variable $t$, is associated with the movement of the platform and therefore often denoted by slow time. The range variation of a point target over time is directly related to the azimuth phase by $\varphi(t)=-4 \pi r(t) / \lambda$, i.e., the phase variation has

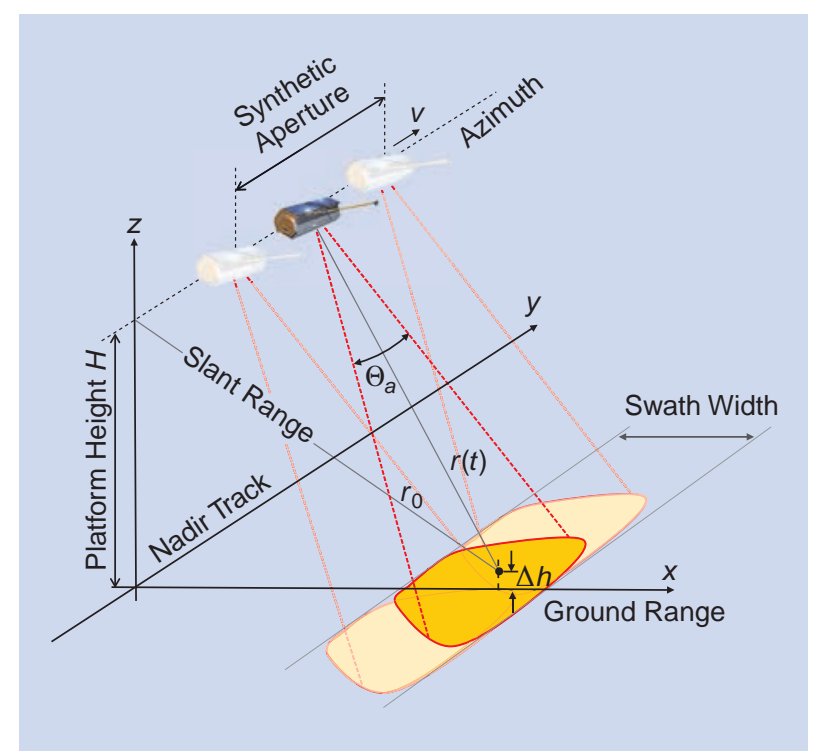

FIGURE 2. Illustration of the SAR imaging geometry. $r_{0}$ stands for the shortest approach distance, $\Theta_{a}$ for the azimuth beamdwidth and $v$ for the sensor velocity. also a parabolic behavior (the factor $4 \pi$ is due to the twoway range measurement of the SAR system). Note that the quadratic approximation in (3) is done for the sake of simplicity. Accurate SAR data processing takes into account the complete phase history without any approximation [8], [9].

Being an imaging radar requires a two-dimensional resolution. The slant-range resolution $\delta_{r}$ is inversely proportional to the system bandwidth according to $\delta_{r}=c_{0} / 2 B_{r}$, where $c_{0}$ is the speed of light. The azimuth resolution $\delta_{a}$ is provided by the construction of the synthetic aperture, which is the path length during which the radar receives echo signals from a point target. The beamwidth of an antenna of length $d_{a}$ can be approximated by $\Theta_{a}=\lambda / d_{a}$. From Fig. 2 it can be seen that the corresponding synthetic aperture length is given through $L_{s a}=\Theta_{a} \cdot r_{0}=\lambda r_{0} / d_{a}$. A long synthetic aperture is favorable since it results in a narrow virtual beamwidth $\Theta_{s a}=\lambda / 2 L_{s a}$ (again, the factor 2 appears because of the two-way path from transmission to reception) and a high azimuth resolution:

$$
\delta_{a}=r_{0} \Theta_{s a}=r_{0} \frac{\lambda}{2 L_{s a}}=\frac{d_{a}}{2} .
$$

The above equation suggests that a short antenna yields a fine azimuth resolution. This appears surprising on the first view. However, it becomes immediately clear if one considers that a radar with a shorter antenna "sees" any point on the ground for a longer time (the illumination time can be approximated by $T_{i l l} \approx \lambda r_{0} / v d_{a}$ ), which is equivalent to a longer virtual antenna length and thus a higher azimuth resolution.

The received echo signal data form a two-dimensional data matrix of complex samples, where each complex sample is given by its real and imaginary part, thus representing an amplitude and phase value. The first dimension corresponds to the range direction (or fast time); a range line consists of the complex echo signal samples after being amplified, down converted to base band, digitized and stored in memory. The radar acquires a range line whenever it travels a distance $v \cdot P R I$ thus forming the second dimension of the data matrix, known as azimuth or slow-time. The very nature of SAR is that the return echoes from the illuminated scene are sampled both in fast time (range) and slow time (azimuth).

Unlike optical sensors, visualizing raw SAR data does not give any useful information on the scene. It is only after signal processing that an image is obtained, as shown in Fig. 3 which summarizes the basic SAR processing steps. In a very simplified way, the complete processing can be understood as two separate matched filter operations along the range and azimuth dimensions. The first step is to compress the transmitted chirp signals to a short pulse. Instead of performing a convolution in the time domain, a multiplication in the frequency domain is adopted due to the much lower computational load. Thus, each range line is multiplied in the frequency 
domain by the complex conjugate of the spectrum of the transmitted chirp; the result is a range compressed image, which reveals only information about the relative distance between the radar and any point on the ground. Azimuth compression follows the same basic reasoning, i.e., the signal is convolved with its reference function, which is the complex conjugate of the response expected from a point target on the ground. Considering an elemental scatterer at range $r(t)$ given in (3), the azimuth signal can be modeled by [9]

$$
s_{a}(t)=A \sqrt{\sigma_{0}} \exp \left(\mathrm{i} \varphi^{\text {scatt }}\right) \exp \left(-\mathrm{i} \frac{4 \pi}{\lambda} r(t)\right),
$$

where $A$ accounts for the dependency of the received signal on system parameters such as transmit power and losses, and the antenna pattern weighting as a function of the azimuth and elevation angles; the radar cross section is given by $\sigma_{0}$ and $\varphi^{\text {scatt }}$ is the scattering phase; $4 \pi r(t) / \lambda$ describes the azimuth phase variation due to the changing distance; and $\mathrm{i}$ is the imaginary unit, i.e., $\mathrm{i}=\sqrt{-1}$.

Interestingly, the frequency variation of the azimuth signal turns out to be similar to that in the range domain, i.e., a linear frequency modulated signal (azimuth chirp). This becomes clear when substituting the approximation (3) into the last exponent in (5) and computing the instantaneous azimuth frequency as the time differentiation of the phase

$$
f_{D}=-\frac{1}{2 \pi} \frac{\partial}{\partial t} \frac{4 \pi}{\lambda} r(t)=-\frac{2 v^{2}}{\lambda r_{0}} t
$$

which varies linearly with time at an azimuth-frequency rate inversely proportional to the slant range. The azimuth frequency is also called Doppler frequency in analogy to the well-known Doppler effect. Fig. 3 shows the basic steps of SAR signal processing, where the range reference function is dependent only on the transmitted chirp waveform whereas the azimuth reference function depends on the geometry and is adapted to the range.

The SAR image is most commonly displayed in terms of intensity values such that each image pixel gives an indication of the reflectivity of the corresponding point on the ground. This involves two additional steps applied on the output of the processor: calibration and geocoding. Here the calibration ensures that the intensity value actually represents the sigma zero $\left(\sigma_{0}\right)$ value of the reflectivity, i.e., the radar cross section normalized to area. Proper calibration is a non-trivial task involving both internal instrument calibration as well as external SAR calibration using targets of known reflectivity [24]. The geocoding on the other hand ensures that the location of any pixel in the SAR image is directly associated to the position on the ground. Typically SAR images are geometrically distorted. The reason for this is that the radar only measures the projection of

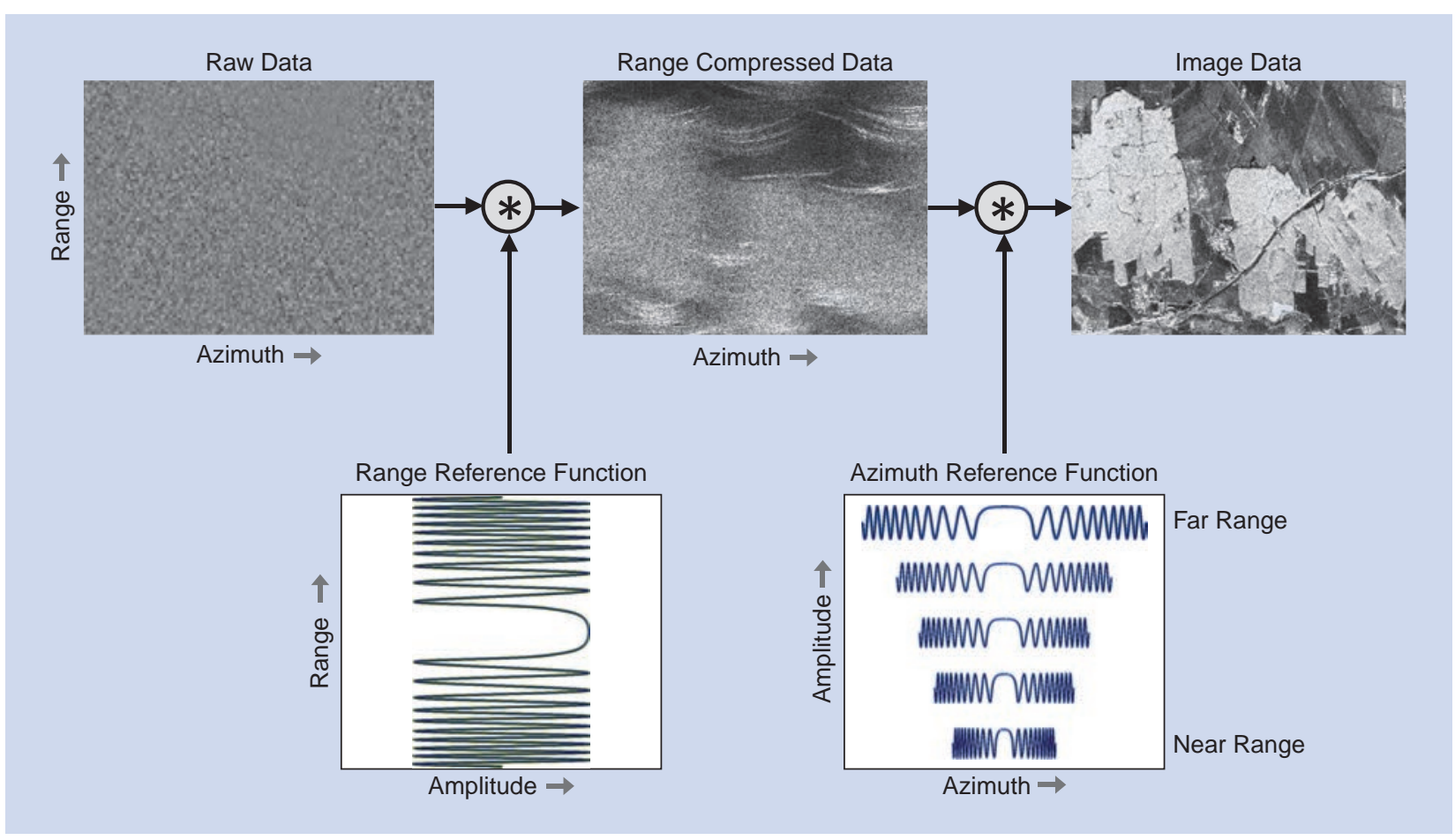

FIGURE 3. Summary of SAR processing steps where the range compressed data result from a convolution of the raw data with the range reference function. In a second step the azimuth compression is performed through a convolution with the azimuth reference function, which changes from near to far range. Here the "*" represents the convolution operation. 
a three-dimensional scene on the radar coordinates slantrange and azimuth. This causes effects such as shadow for areas hidden from the radar illumination as well as foreshortening and layover manifested by a stretch and compression of sloped terrain.

A quantitative measure of the signal processing quality is possible by investigating the impulse response function (IRF). This is basically the two-dimensional complex image that would be obtained from a scene consisting of a single point-like scatterer. The IRF is most often computed based on simulated data, or derived analytically, but it can also be measured when strong scatterers are present in the imaged scene. Specifically the range/azimuth resolution (taken as the respective halfpower width of the IRF) and side-lobe levels are of interest.

Analyzing the IRF reveals that the phase, especially in azimuth as given by (5), is crucial for the correct focusing. This has a strong impact on the instrument hardware, which is required to have a high phase stability, i.e., to be coherent during the data acquisition. This is nowadays not an issue due to the availability of ultra-stable oscillators.

Most SAR raw data properties can be described taking into account simple geometrical properties. Of these Range Cell Migration (RCM) is a property originating from the fact that the distance between the radar and any fixed point

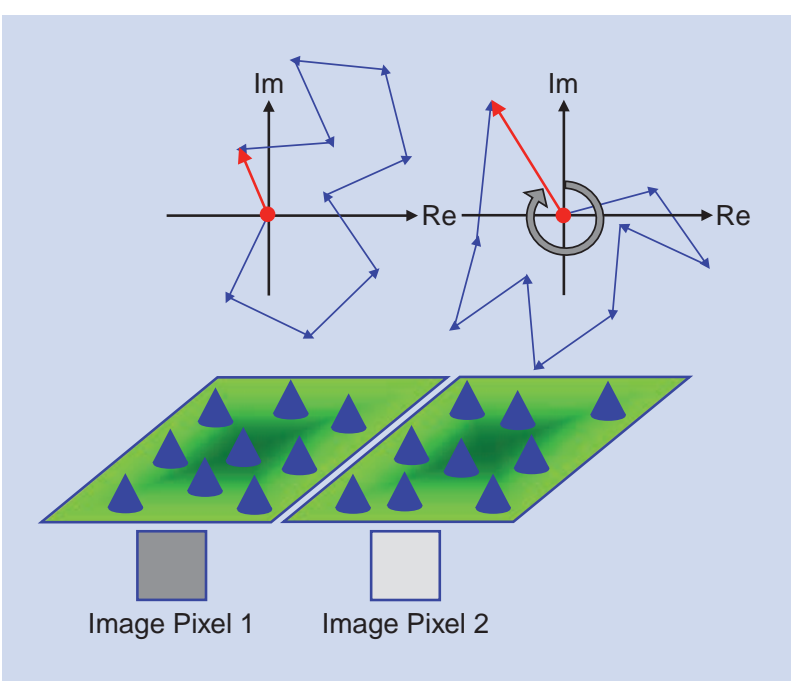

FIGURE 4. Speckle occurs in SAR images due to the coherent sum of many elemental scatterers within a resolution cell. The two parallelograms show the distribution of the scatterers in each resolution cell and the resulting amplitude and phase values. Due to the random distribution of the scatterers, the resulting intensity and phase change from pixel to pixel, showing an exponential and uniform distribution, respectively [5]. Speckle appears in areas with distributed scatterers where the radar wavelength is comparable to the surface roughness. on the ground is changing within the synthetic aperture time. This distance change is obtained from (3) by subtracting the constant $r_{0}$ and is given by

$$
R C M(t)=\sqrt{r_{0}^{2}+(v t)^{2}}-r_{0} \approx \frac{(v t)^{2}}{2 r_{0}} .
$$

The RCM can be observed through the curvature of the range compressed responses in Fig. 3. If not corrected, RCM causes an azimuth defocusing when $R C M_{\text {max }}=R C M\left(t=T_{\text {ill }} / 2\right)>\delta_{r} / 2$ because in this case the point target energy is distributed over several range cells. The fact that the range migration is range-variant, i.e., the curvature depends on $r_{0}$, makes SAR focusing a two-dimensional space-variant problem, and hence the data need to be correlated with a non-stationary two-dimensional reference function, making the accurate correction of RCM the most challenging aspect of SAR focusing. In the beginnings of digital processing and especially in the 90s, the efficient correction of the RCM was an intense research topic, resulting in several approaches, of which the most commonly known are those based on $\omega-k$ (or "wavenumber domain") processors [25], [26], range-Doppler algorithms [27]-[29], as well as chirp scaling approaches [30], [31]. Detailed analyses and comparisons of these processors, as well as further focusing approaches, can be found in several books [2], [8], [9], [32].

A particular effect to be observed in SAR images is the so-called speckle, which is caused by the presence of many elemental scatterers with a random distribution within a resolution cell. The coherent sum of their amplitudes and phases results in strong fluctuations of the backscattering from resolution cell to resolution cell. Consequently, the intensity and the phase in the final image are no longer deterministic, but follow instead an exponential and uniform distribution, respectively [5]. The total complex reflectivity for each resolution cell is given by

$$
\Phi=\sum_{i} \sqrt{\sigma_{i}} \exp \left(\mathrm{i} \varphi_{i}^{\text {scatt }}\right) \cdot \exp \left(-\mathrm{i} \frac{4 \pi}{\lambda} r_{0, i}\right),
$$

where $i$ is the number of elemental scatterers within the resolution cell. Speckle is indeed a physical measurement of the resolution cell structure at sub-resolution level. Although it is commonly referred to as noise, speckle cannot be reduced by increasing the transmit signal power, since it has a multiplicative character, i.e., its variance increases with its intensity. To mitigate speckle a technique known as multi-look is utilized, which is basically a non-coherent averaging of the intensity image [2], [5]. Although multilook causes a degradation in the image resolution, it greatly improves the interpretability of the SAR image as it can be seen in Figures 5(b)-5(d). Also the effect of speckle tends to weaken for very high-resolution systems, since the number of elemental scatterers within a resolution cell decreases.

One of the key issues of SAR is the signal sampling. In range, the sampling rate of the analog-to-digital converter 
must be larger than the transmitted chirp bandwidth (assuming I/Q demodulation) according to Shannon's sampling theorem. The same is true in azimuth. However, in this case the sampling rate is equal to the PRF which must be larger than the signal Doppler bandwidth. This can be stated as

$$
P R F \geq B_{D}=\frac{2 v}{d_{a}}
$$

which can be rearranged in terms of the spatial sample spacing $v \cdot P R I \leq d_{a} / 2$, i.e., at least one sample (pulse transmission event) is required whenever the platform has moved by half of the antenna length.

When determining the required sampling rate it turns out that improving the range resolution increases the data rate and volume on board the satellite. In azimuth the situation is more complex due to the interrelation between various parameters. Here, improving the azimuth resolution means a higher Doppler bandwidth of the received echo signal and consequently a higher sampling, which in this case means increasing the PRF. This, however, reduces the echo window length and by this the time available for receiving the echoes, which means a reduced swath width. It is seen that fine azimuth resolution and wide swath contradict each other and cannot be obtained simultaneously; at least not with conventional single-channel SAR (cf. Section VII).

Current SAR systems are capable of operating in different imaging modes by controlling the antenna radiation pattern. For a planar antenna this is done by dividing the antenna into sub-apertures and controlling the phase and amplitude of each sub-aperture through transmit/receive modules (TRM). Typically a few hundred TRMs are employed, with their settings controlled by software. The most fundamental mode is the Stripmap operation, where the pattern is fixed to one swath, thus imaging a single continuous strip as shown in Fig. 6(a). If a wider swath is required, the system can be operated in the ScanSAR mode. Here the antenna elevation pattern is successively steered to different elevation

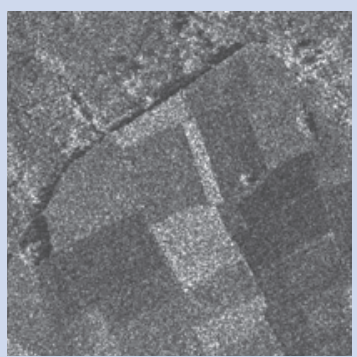

(a)

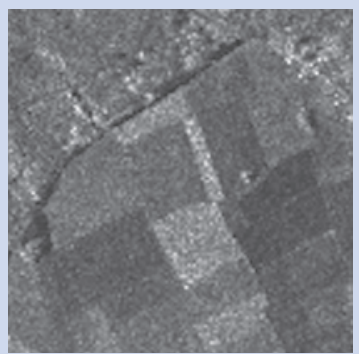

(c)

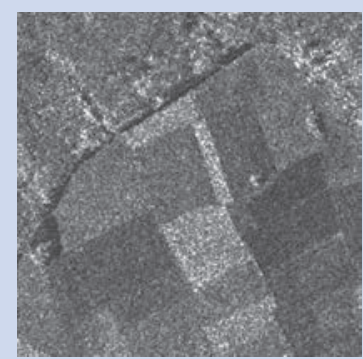

(b)

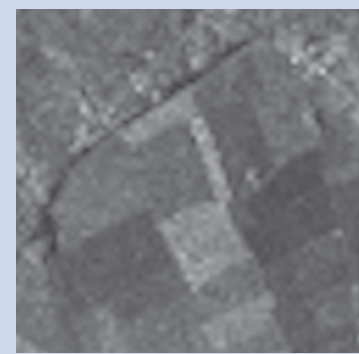

(d)
FIGURE 5. The effect of speckle can be reduced through multi-look in azimuth and range which also worsens the resolution as seen in the multi-looked SAR images above. (a) Without multi-look. (b) $2 \times 2$ multi-look. (c) $4 \times 4$ multi-look. (d) $8 \times 8$ multi-look.

angles corresponding to multiple sub-swaths as shown in Fig. 6(b). Each sub-swath is illuminated by multiple pulses but for a shorter time than in the Stripmap case. The timing is adjusted such that the time-varying elevation patterns repeat cyclically the imaging of multiple continuous sub-swaths. After appropriate processing this yields a wide-swath SAR image, however, the azimuth resolution is degraded when compared to the Stripmap mode. If a better azimuth resolution is required, the Spotlight mode is utilized. Here the antenna pattern is steered in azimuth towards a fixed point to illuminate a given region as shown in Fig. 6(c). The long illumination

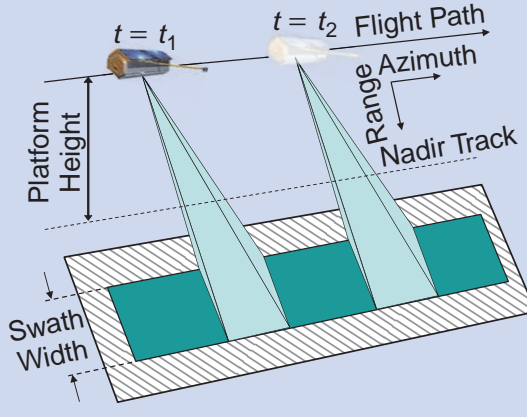

(a)

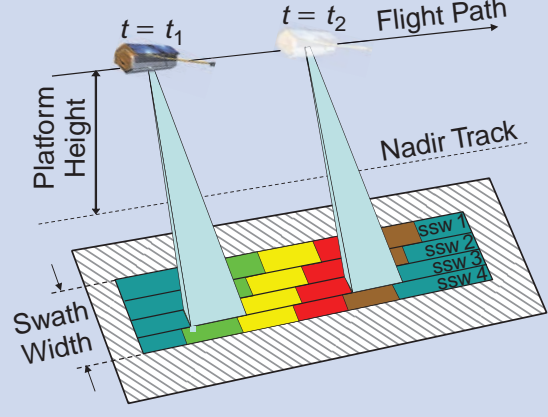

(b)

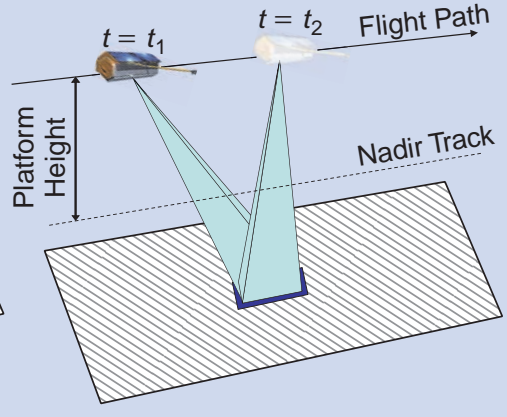

(c)

FIGURE 6. Illustration of different SAR operation modes which are used to increase the swath width (ScanSAR) or improve the azimuth resolution (Spotlight) compared to the Stripmap mode. (a) Stripmap. (b) ScanSAR. (c) Spotlight. 


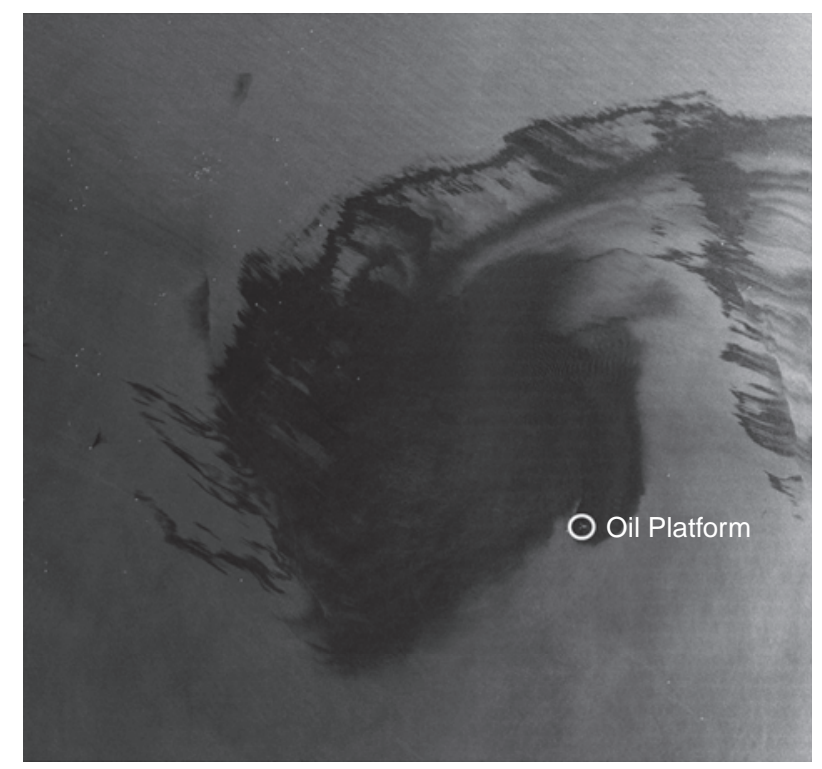

FIGURE 7. Oil pollution over the Gulf of Mexico acquired on April 30, 2010 (just 10 days after the explosion of "Deepwater Horizon," leading to the largest offshore oil spill in U.S. history). Oil spills appear as dark areas in radar images because of the attenuation of the capillary waves, leading to a smoother ocean surface and decrease of the backscattered signal. Capillary waves are wind-caused ripplewaves with a wavelength less than a few centimeters. Image size: ca. $100 \mathrm{~km} \times 100 \mathrm{~km}$, acquired by TerraSAR-X in ScanSAR mode.

time results in an increased synthetic aperture length and consequently in a better resolution. However, the Spotlight mode does not image a continuous swath but rather individual patches along the radar flight path. Other

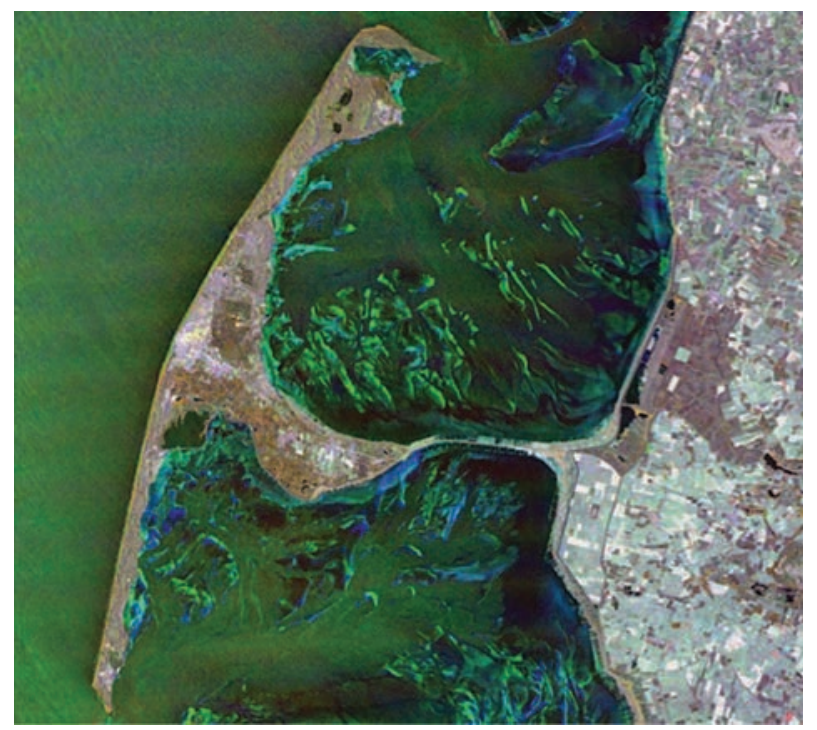

FIGURE 8. Multi-temporal acquisition of Sylt, the most northern island of Germany, acquired by TerraSAR-X on the 22nd, 24th and 27th of October, 2007. Each image has been associated to a different color channel (green, blue and red, respectively). While the changes during the 5-day time span over land areas are relatively small, the changes in the Wadden Sea caused by the ocean tide are clearly seen. imaging modes exist, such as TOPS [33] or the wave mode, each one improving certain performance parameters, but at the expense of others. It turns out that there exist fundamental limits on single-channel SAR such that improving azimuth resolution results in a degradation of the swath width and vice versa. A system operated such that it violates these limits will result in image ambiguities, i.e., scene features which appear at multiple or wrong positions in the radar image. These limitations can be overcome through multi-channel digital beamforming techniques, which will be introduced in Section VII.

In the beginning, SAR images were mainly interesting from the science and engineering point of view. The last years have marked an important transition in the application of SAR. This has changed and nowadays the public awareness of the usefulness of radar remote sensing beyond science is much higher. As an example, radar satellites are predestined to perform disaster monitoring, mitigation and damage assessment due to their allweather day-and-night imaging capability. Fig. 7 shows a SAR image of oil pollution over the Gulf of Mexico taken 10 days after the explosion of the oil drilling unit "Deepwater Horizon."

Another example are multi-temporal acquisitions of the German island Sylt. Fig. 8 shows the changes during a 5-day time span. Due to the large extent of the Wadden Sea (ca. 10,000 square kilometers) long-term time series of radar images offer important information to study the morphology, sediments and habitats in this area, which is being particularly affected by sea level rise.

\section{POLARIMETRY}

SAR polarimetry is a widely used technique for the derivation of qualitative and quantitative physical information for land, snow and ice, ocean and urban applications based on the measurement and exploration of the polarimetric properties of man-made and natural scatterers [1], [15], [34], [35]. Measuring the full scattering matrix allows to build up a powerful observation space sensitive to shape, orientation and dielectric properties of the scatterers and allows the development of physical models for the identification and/or separation of scattering mechanisms occurring inside the same resolution cell [1].

\section{A. POLARIMETRIC DESCRIPTION OF THE SCATTERER}

The basic concept of SAR polarimetry is given by the $2 \times 2$ complex scattering matrix that describes the transformation of the two-dimensional transmitted (e.g., incidence) plane wave vector $\vec{E}^{t}$ into the received (e.g., scattered) wave vector $\vec{E}^{r}$ (two-dimensional in the far field of the scatterer) performed by the scatterer [15], [35], [36]

$$
\begin{gathered}
\vec{E}^{r}=\frac{\exp (-\mathrm{i} k r)}{r}[S] \vec{E}^{t^{*}} \\
{\left[\begin{array}{c}
E_{H}^{r} \\
E_{V}^{r}
\end{array}\right]=\frac{\exp (-\mathrm{i} k r)}{r} \cdot\left[\begin{array}{ll}
S_{H H} & S_{H V} \\
S_{V H} & S_{V V}
\end{array}\right]\left[\begin{array}{c}
E_{H}^{t} \\
E_{V}^{t}
\end{array}\right]^{*}}
\end{gathered}
$$


The elements of [S] are the four complex scattering amplitudes $S_{I J}=\left|S_{I J}\right| \exp \left(\mathrm{i} \varphi_{I J}\right)$ where the subscripts horizontal $(\mathrm{H})$ or vertical $(\mathrm{V})$ indicate associated received and transmitted polarization. The factor $\exp (-\mathrm{i} k r) / r$, where $k=2 \pi / \lambda$ is the wave number, expresses the phase shift and attenuation for a spherical wave of a radius that equals the distance between the scatterer and the radar. In SAR interferometry this absolute phase term is used to estimate the three-dimensional location of the scatterer. In SAR polarimetry the absolute phase is in most cases neglected and only the relative phases $\exp \left(\mathrm{i}\left(\varphi_{I J}-\varphi_{M N}\right)\right)$ between the matrix elements are considered.

The scattering matrix can be measured by transmitting in two orthogonal polarizations on a pulse-to-pulse basis and receiving the scattered waves in two orthogonal polarizations (commonly the same basis as used for transmission). Most polarimetric systems operate in the linear $\mathrm{H}-\mathrm{V}$ basis: By transmitting a $\mathrm{H}$ polarized wave (i.e., $\vec{E}^{t} /\left|\vec{E}^{t}\right|=[1,0]^{T}$ where the superscript $T$ indicates the transpose operation) and receiving in $\mathrm{H}$ (i.e., $\vec{E}^{r} /\left|\vec{E}^{r}\right|=[1,0]^{T}$ ) and V (i.e., $\vec{E}^{r} /\left|\vec{E}^{r}\right|=[0,1]^{T}$ ) polarization the $S_{H H}$ and $S_{V H}$ elements are measured. The two remaining coefficients $S_{V H}$ and $S_{V V}$ are measured in a second step by transmitting a $\mathrm{V}$ polarized wave (i.e., $\vec{E}^{t} /\left|\vec{E}^{t}\right|=[0,1]^{T}$ ) and receiving in $\mathrm{H}$ and $\mathrm{V}$. However, $[S]$ can be measured also by using any other basis of orthogonal polarizations, as for example left and right circular polarizations as well as by using different bases for transmit and receive (e.g., left-right circular polarization on transmit and linear $\mathrm{H}-\mathrm{V}$ polarization on receive).

In this sense it is important to note that the information content of the $[S]$ matrix is independent of the basis used for its measurement, but its representation of course depends on the chosen reference frame (i.e., the bases used for the measurement). Accordingly, once the full scattering matrix is measured, any arbitrary complex scattering amplitude can be reconstructed as a linear combination of the elements of the measured scattering matrix. This is the great advantage of fully polarimetric radar systems over conventional single- or dual-polarized configurations.

In monostatic configurations, where receiver and transmitter are co-located, the $[S]$ matrix becomes symmetric, i.e., $S_{H V}=S_{V H}=S_{X X}$, for all reciprocal scattering media. In this case, and ignoring the absolute phase, the number of independent parameters in $[S]$ is reduced to five: Three amplitudes and two relative phases. In the bistatic case, where receiver and transmitter are spatially separated, $S_{H V} \neq S_{V H}$ and $[S]$ contains seven independent parameters: Four amplitudes and three relative phases. While bistatic polarimetry is widely used in optics, in SAR remote sensing the majority of the polarimetric systems is operated in a monostatic mode. Very few experiments have been successful in collecting fully polarimetric bistatic data providing some very first insight in the physical interpretation of bistatic SAR polarimetry [37]. In the following, all formulations will refer to the monostatic case.

The set of observables derived from the scattering matrix and used in remote sensing applications is summarized in Table 3.

The scattering matrix is able to completely describe deterministic (point-like) scatterers that change the polarization of the incident wave, but fails to describe the depolarization of the incident wave as it happens in the case of distributed scatterers. Distributed scatterers are considered to be composed of a large number of randomly distributed deterministic scatterers (see(8)). The measured scattering matrix consists then of the coherent superposition of the individual scattering matrices of all scattering centers within the resolution cell. In order to fully describe the polarimetric scattering behavior of distributed scatterers a second-order statistical formalism is required.

The most common formalism to fully characterize distributed scatterers is the $3 \times 3$ coherency [T] (or covariance $[C]$ ) matrix defined by the outer product of a three-dimensional scattering vector in the Pauli $\vec{k}_{P}$ (or lexicographic $\vec{k}_{L}$ ) formulation:

$$
\vec{k}_{P}=\frac{1}{\sqrt{2}}\left[S_{H H}+S_{V V}, S_{H H}-S_{V V}, 2 S_{X X}\right]^{T},
$$

and the coherency matrix is given by (12), shown at the bottom of the page, where the superscript + indicates the conjugate transpose operation. While the lexicographic formulation is more appropriate for system related considerations, the Pauli formulation is of advantage when it comes to the interpretation of scattering processes [15].

Both matrices are by definition hermitian positive semi definite, have the same real non-negative eigenvalues but different orthonormal eigenvectors, and are in general of full rank 3. With respect to the physical information content the rank of $[T]$ (or $[C]$ ) expresses the number of independent scattering contributions in which $[T]$ (or $[C]$ ) can be decomposed. In the most general case both matrices contain nine

$$
[T]=<\vec{k}_{P} \cdot \vec{k}_{P}^{+}>=\frac{1}{2}\left[\begin{array}{ccc}
<\left|S_{H H}+S_{V V}\right|^{2}> & <\left(S_{H H}+S_{V V}\right)\left(S_{H H}-S_{V V}\right)^{*}> & 2<\left(S_{H H}+S_{V V}\right) S_{X X}^{*}> \\
\left\langle\left(S_{H H}-S_{V V}\right)\left(S_{H H}+S_{V V}\right)^{*}>\right. & <\left|S_{H H}-S_{V V}\right|^{2}> & 2<\left(S_{H H}-S_{V V}\right) S_{X X}^{*}> \\
2<S_{X X}\left(S_{H H}+S_{V V}\right)^{*}> & 2<S_{X X}\left(S_{H H}-S_{V V}\right)^{*}> & 4<\left|S_{X X}\right|^{2}>
\end{array}\right]
$$


TABLE 3. POLARIMETRIC RADAR OBSERVABLES AND PARAMETERS DERIVED FROM THE ELEMENTS

OF THE SCATTERING MATRIX [S] [5], [15], [38]-[41].

\section{$\mathbf{2} \times \mathbf{2}$ SINCLAIR MATRIX \\ Scattering amplitude (complex) \\ and scattering power}

Total power

Amplitude ratios

Polarimetric phase differences

Helicity

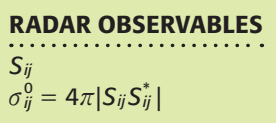

$T P=\left|S_{H H}\right|^{2}+2\left|S_{X X}\right|^{2}+\left|S_{V V}\right|^{2}$

$\sigma_{H H}^{0} / \sigma_{W}^{0}, \sigma_{X X}^{0} / \sigma_{W}^{0}, \sigma_{X X}^{0} /\left(\sigma_{H H}^{0}+\sigma_{V V}^{0}\right)$

$\varphi_{H H V V}=\varphi_{H H}-\varphi_{V V}$

$\mathrm{Hel}=\left|S_{L L}\right|-\left|S_{R R}\right|$

\section{APPLICATION EXAMPLES}

Classification/segmentation (texture based)

Change detection (multitemporal analysis)

Glacier velocities (feature tracking)

Ocean wave and wind mapping

Coherent scatterers

Classification/segmentation

Feature tracking

Dry/wet snow separation

Soil moisture and surface roughness estimation (bare surfaces)

Thin sea ice thickness

Crop types identification

Forest/nonforest classification

Man-made target identification independent parameters in form of six independent matrix elements: Three real diagonal power elements and three off-diagonal complex cross-correlations.

Symmetry assumptions about the distribution of elementary scatterers within the resolution cell simplify the scattering problem and reduce the number of independent parameters of $[T]$ (or $[C]$ ) allowing qualitative and quantitative conclusions about the scattering behavior [35], [42], [43]. Besides reciprocity, three special cases of symmetry are important in radar remote sensing applications: Reflection, rotation and azimuthal symmetry. Reflection symmetric media are characterized by a symmetry plane that contains the line-of-sight so that for any scatterer located at one side of the plane a mirrored scatterer at the other side of the plane exists. In this case the correlation between the co- and crosspolarized elements becomes zero. The resulting $[T]$ matrix contains only five independent parameters in form of three real diagonal elements and one single non-zero complex off-diagonal element (i.e., the correlation between the copolarized elements). The majority of natural distributed scatterers is reflection symmetric. In the case of rotation symmetry, the spatial distributions of elementary scatterers do not change when rotated about the line-of-sight (LOS) axis. Accordingly, the scattering behavior of such media is invariant under the line-of-sight rotations and the resulting coherency matrix contains only three independent parameters in form of two independent real diagonal elements and one non-zero imaginary off-diagonal element. This is typical for gyrotropic random media, as given for example by a random distribution of helices. When both, reflection and rotation symmetry applies, the medium is said to be azimuthally symmetric: All planes including the line-of-sight are reflection symmetry planes. Consequently, all three offdiagonal elements of the coherency matrix become zero, and only two diagonal elements are independent, the number of independent parameters reduces to 2. This is the case for volumes consisting of random distributions of ellipsoids.

Compared to the elements of the scattering matrix $[S]$, the coherency (or covariance) matrix elements have a reduced resolution because of the spatial averaging (i.e., multi-looking), indicated by $\langle\cdots\rangle$, performed for the formation of $[T]$. Despite the higher radiometric resolution achieved, this loss in resolution may be critical especially for point scatterers but also for applications on distributed scatterers. This can be partially compensated by using adaptive (edge and point scatterers preserving) filters to perform the required multi-looking [15], [44].

The set of observables derived from the coherency (or covariance) matrix contains, in addition to the observables derived from $[S]$, the correlation coefficients between different polarizations, which are given by

$$
\begin{aligned}
\gamma_{H H V V} & =\frac{<\left|S_{H H} S_{V V}^{*}\right|>}{\sqrt{<\left|S_{H H} S_{H H}^{*}\right|><\left|S_{V V} S_{V V}^{*}\right|>}} \\
\gamma_{L L R R} & =\frac{<\left|S_{L L} S_{R R}^{*}\right|>}{\sqrt{<\left|S_{L L} S_{L L}^{*}\right|><\left|S_{R R} S_{R R}^{*}\right|>}}
\end{aligned}
$$

where $S_{L L}$ and $S_{R R}$ are the circular left-left and circular right-right (complex) scattering amplitudes that can be expressed as a linear combination of the scattering amplitudes of the scattering matrix $[S]$ measured in the $H-V$ basis (cf. (10)) as

$$
\begin{aligned}
& S_{L L}=\frac{1}{2}\left(S_{H H}+2 \mathrm{i} S_{X X}-S_{V V}\right) \\
& S_{R R}=\frac{1}{2}\left(S_{V V}+2 \mathrm{i} S_{X X}-S_{H H}\right) .
\end{aligned}
$$

and whose application examples are summarized in Table 4.

\section{B. INTERPRETATION AND DECOMPOSITION OF SCATTERING PROCESSES}

The main objective of scattering decomposition approaches is to break down the polarimetric backscattering signature of distributed scatterers which is in general given by the superposition of different scattering contributions inside the resolution cell into a sum of elementary scattering contributions. The most common elementary scattering 
mechanisms used are surface, dihedral and volume (or multiple) scattering. Scattering decompositions are widely applied for interpretation, classification, segmentation and/or as a pre-processing step for scattering parameter inversion. In general, the decompositions of second-order scattering matrices (i.e., $[T]$ or $[C]$ ) are rendered into two classes: Eigenvector and eigenvalue based decompositions and model-based decompositions.

An extended review of scattering decompositions can be found in [15]. The basic concepts of the eigenvector and model-based decompositions are described in the next section.

1) Eigenvector and Eigenvalue based Decomposition: Since the coherency matrix $[T]$ is hermitian positive semi-definite, it can always be diagonalized by a unitary similarity transformation of the form [15], [35]

$$
[T]=[U][\Lambda][U]^{-1},
$$

where

$$
\Lambda=\left[\begin{array}{ccc}
\lambda_{1} & 0 & 0 \\
0 & \lambda_{2} & 0 \\
0 & 0 & \lambda_{3}
\end{array}\right], \quad[U]=\left[\begin{array}{lll}
e_{11} & e_{12} & e_{13} \\
e_{21} & e_{22} & e_{23} \\
e_{31} & e_{32} & e_{33}
\end{array}\right] .
$$

$[\Lambda]$ is the diagonal eigenvalue matrix with elements the real nonnegative eigenvalues, $0 \leq \lambda_{3} \leq \lambda_{2} \leq \lambda_{1}$ and $[U]=\left[\begin{array}{lll}\vec{e}_{1} & \vec{e}_{2} & \vec{e}_{3}\end{array}\right]$ is the unitary eigenvector matrix with columns the corresponding orthonormal eigenvectors $\vec{e}_{1}, \vec{e}_{2}$ and $\vec{e}_{3}$. Each of them can be parametrized in terms of five angles [15], [35]

$$
\vec{e}_{i}=\left[\begin{array}{c}
\cos \alpha_{i} e^{i \Psi_{1 i}} \\
\sin \alpha_{i} \cos \beta_{i} e^{i \Psi_{2 i}} \\
\sin \alpha_{i} \sin \beta_{i} e^{i \Psi_{3 i}}
\end{array}\right] .
$$

that-as it will be discussed later-characterize the associated scattering mechanism.

The idea of the eigenvector approach is to use the diagonalization of the coherency matrix [T] of a distributed scatterer, which is in general of rank 3 , as a decomposition into the non-coherent sum of three independent (i.e., orthogonal) coherency matrices $\left[T_{i}\right]$

$$
\begin{aligned}
{[T] } & =[U][\Lambda][U]^{-1}=\left[T_{1}\right]+\left[T_{2}\right]+\left[T_{3}\right] \\
& =\lambda_{1} \cdot\left(\vec{e}_{1} \cdot \vec{e}_{1}^{+}\right)+\lambda_{2} \cdot\left(\vec{e}_{2} \cdot \vec{e}_{2}^{+}\right)+\lambda_{3} \cdot\left(\vec{e}_{3} \cdot \vec{e}_{3}^{+}\right) .
\end{aligned}
$$

The $\left[T_{i}\right]$ matrices are of rank 1 implying a deterministic scattering contribution, characterized by a single scattering matrix. There are two important statistical parameters arising directly from the eigenvalues of the coherency matrix. The first one is the polarimetric scattering entropy $H$ defined by the logarithmic sum of the eigenvalues of $[T]$

$$
H=-\sum_{i=1}^{3} p_{i} \log _{3} p_{i}, p_{i}=\frac{\lambda_{i}}{\sum_{j=1}^{3} \lambda_{j}}
$$

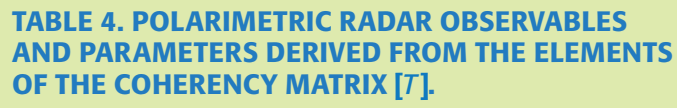

TABLE 4. POLARIMETRIC RADAR OBSERVABLES

AND PARAMETERS DERIVED FROM THE ELEMENTS

OF THE COHERENCY MATRIX [T].

$\mathbf{3} \times \mathbf{3}$ BACKSCATTERING MATRIX

Correlation coefficient

HV basis $\left(\gamma_{H н V V}\right)$

Correlation coefficient

LR basis $(\gamma$ LLRR $)$

\section{APPLICATION EXAMPLES} Crop phenology classification Dielectric constant estimation of bare soils/surfaces

Surface roughness estimation (bare surfaces) where $p_{i}$ expresses the appearance probability for each contribution. The entropy ranges from 0 to 1 and can be interpreted as a measure of the randomness of the scattering process, or in other words, it expresses the number of effective scattering processes in which $[T]$ can be decomposed by means of (18). An entropy of 0 indicates a rank $1[T]$ matrix with only one nonzero eigenvalue, i.e., $\lambda_{2}=\lambda_{3}=0$, implying a non-depolarizing scattering process described by a single scattering matrix. At the other extreme an entropy of 1 indicates the presence of three equal nonzero eigenvalues, i.e., $\lambda_{1}=\lambda_{2}=\lambda_{3}$ and characterizes a random noise scattering process, which depolarizes completely the incidence wave regardless of its polarization. However, most distributed natural scatterers lie in between these two extreme cases, having intermediate entropy values.

The second parameter is the polarimetric scattering anisotropy defined as the normalized difference of the second and third eigenvalues

$$
A=\frac{\lambda_{2}-\lambda_{3}}{\lambda_{2}+\lambda_{3}}
$$

A ranges also from 0 to 1 and expresses the relation between the secondary scattering processes. For a deterministic scatterer with entropy $H=0$ the anisotropy is defined as zero, $A=0$. The same is the case for a completely depolarizing scatterer with $H=1$. For scatterers characterized by intermediate entropy values, a high anisotropy indicates the presence of only one strong secondary scattering process. In this sense, the anisotropy provides complementary information to the entropy and facilitates the interpretation of the scatterer. The great advantage of these two parameters arises from the invariance of the eigenvalue problem under unitary transformations: The same scatterer leads to the same eigenvalues and consequently to the same entropy and anisotropy values independent of the basis used to measure the corresponding scattering matrix.

The physical and geometrical interpretation of the scattering mechanisms represented by $\left[T_{1}\right],\left[T_{2}\right]$ and $\left[T_{3}\right]$ is given by the corresponding eigenvectors. For each eigenvector the scattering alpha angle $\alpha_{i}=\arccos \left(\left|e_{1 i}\right|\right)$ ranges between 0 and 90 degrees and is associated to the type of corresponding scattering mechanism: $0^{\circ} \leq \alpha_{i} \leq 30^{\circ}$ corresponds in general to surface scattering processes, $40^{\circ} \leq \alpha_{i} \leq 50^{\circ}$ to dipol-like scattering behavior and finally $60^{\circ} \leq \alpha_{i} \leq 90^{\circ}$ 
TABLE 5. POSSIBLE RADAR OBSERVABLES ESTIMATED FROM THE SECOND-ORDER STATISTICAL PARAMETERS AND THEIR RELATION TO THE ENVIRONMENTAL PARAMETERS/ APPLICATION EXAMPLES [15].

SECOND-ORDER

\section{STATISTICAL PARAMETER}

Entropy $(H)$

Anisotropy $(A)$

Alpha angle $(\alpha)$

Beta angle $(\beta)$

\section{APPLICATION EXAMPLES} Segmentation and classification Segmentation and classification Surface roughness estimation

Segmentation and classification Soil moisture estimation (bare surfaces)

Surface slope estimation (azimuth direction) (bare surfaces)

indicates dihedral (or helix type) scattering mechanisms. The beta scattering angle, $\beta_{i}=\arctan \left(\left|e_{3 i}\right| /\left|e_{2 i}\right|\right)$, ranges between $-\pi$ and $\pi$ and describes the line-of-sight rotation (given by $\beta / 2$ ) of the scatterer represented by $\left[T_{i}\right]$. The existence of three such $\alpha_{i}$ and $\beta_{i}$ scattering angles motivates a statistical interpretation in terms of mean scattering angles, given by

$$
\begin{aligned}
& \alpha=p_{1} \alpha_{1}+p_{2} \alpha_{2}+p_{3} \alpha_{3} \\
& \beta=p_{1} \beta_{1}+p_{2} \beta_{2}+p_{3} \beta_{3} .
\end{aligned}
$$

The entropy/(mean) alpha $(H / \alpha)$ space has been used primarily for the initialization of unsupervised classifications [15]. In Table 5 second-order statistical parameters and their corresponding applications are listed.

2) Model-Based Decomposition: Model-based decompositions have been proposed and applied, in order to decompose the individual scattering contribution into a set of predefined elementary scattering contributions [45]-[48].

One of the first model-based decompositions used to fit and to interpret SAR data is the Three-Component Decomposition proposed by A. Freeman and S.L. Durden in 1998 [45]. The model decomposes the measured coherency matrix $[T]$ into a Bragg scattering component (first matrix), a (smooth) dihedral reflection (second matrix), and into a volume of randomly oriented dipole scattering elements (third matrix)

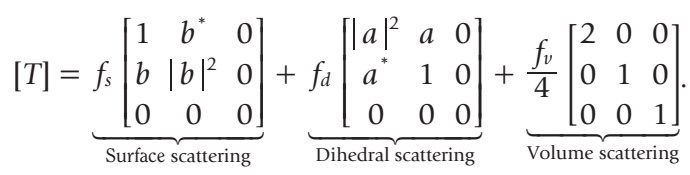

The surface contribution is described by two parameters: The real ratio $b=\left(R_{s}+R_{p}\right) /\left(R_{s}-R_{p}\right)$ and the backscattering contribution $f_{s}=\left|R_{s}-R_{p}\right|^{2}$ where $R_{s}$ and $R_{p}$ are the Bragg scattering coefficients perpendicular and parallel to the incident plane and depend on the dielectric constant of the surface and the incidence angle [15], [35]. Also the

dihedral component is described by two parameters: The scattering amplitude

$$
f_{d}=\frac{1}{2}\left|R_{s h} R_{t h}+R_{s v} R_{t v} \exp (\mathrm{i} \phi)\right|^{2}
$$

and the ratio

$$
a=\frac{R_{s h} R_{t h}-R_{s v} R_{t v} \exp (\mathrm{i} \phi)}{R_{s h} R_{t h}+R_{s v} R_{t v} \exp (\mathrm{i} \phi)} .
$$

The horizontal and vertical Fresnel coefficients for the soil $R_{s h}$ and $R_{s v}$ and for the trunk $R_{t h}$ and $R_{t v}$ depend on the soil (trunk) dielectric constants and the corresponding incidence angle [15], [35]. The volume component $f_{v}$ is often approximated by a cloud of ellipsoids [35]. The phase component $\phi$ accounts for any differential propagation effects in the volume. Hence, the scattering properties of the layer (neglecting multiple scattering interactions) are controlled by three parameters: The electromagnetic density of the volume that affects the scattered power, the shape and the orientation distribution of the volume particles. The exact volume formulation is described in [35], [49]. The scattering power of the surface $P_{s}$, dihedral $P_{d}$ and volume $P_{v}$ component are given by the trace of the corresponding coherency matrices

$$
P_{s}=f_{s} \cdot\left(1+|b|^{2}\right), \quad P_{d}=f_{d} \cdot\left(1+|a|^{2}\right), \quad P_{v}=f_{v} .
$$

The total power is obtained as

$$
\begin{aligned}
P_{\text {tot }} & =P_{s}+P_{d}+P_{v} \\
& =f_{s} \cdot\left(1+|\beta|^{2}\right)+f_{d} \cdot\left(1+|\alpha|^{2}\right)+f_{v} .
\end{aligned}
$$

Eq. (22) leads to an under-determined inversion problem of 5 (3 real and one complex) observables for six (real) unknown parameters. For this, the non-dominant of the two components, either surface or dihedral, is set to a constant value according to the sign of $\operatorname{Re}\left\{\left\langle S_{H H} S_{V V}^{*}\right\rangle\right\}$ after removal of the volume contribution. Surface scattering is dominant if this term is positive otherwise dihedral scattering is dominant.

The described canonical models have been widely used as elements of different decomposition approaches. Beyond this, several modifications have been proposed in order to improve the interpretation of real scattering scenarios by means of more realistic model components [49], [50]. Besides the interpretation of polarimetric data over vegetated terrain, model-based decompositions have been used to initialize and support polarimetric segmentation and classification approaches [15]. Fig. 9 shows on the top the Freeman and Durden decomposition applied on quad-polarimetric L-band data acquired by DLR's E-SAR system over the Oberpfaffenhofen test site. Red, green and blue correspond to $f_{d}, f_{v}$ and $f_{s}$. In a second step, the dominant of the three contributions has been used to classify the scene into three classes: Dihedral, volume and surface like scatterers. These three classes are then used to initialize a statistical Wishart 
classifier able to refine up to 16 classes [15]. The result is shown on the bottom of Fig. 9. More recently, polarimetric decomposition techniques have been successfully used to separate and remove the disturbing vegetation contribution and allow estimation of the soil moisture content on the isolated surface components. Fig. 10 shows the soil moisture maps obtained from polarimetric L-band data acquired at three different dates in the frame of the AGRISAR experiment in 2006 [51]. At the time of the first acquisition in April, the crop layer was still short and light. Its height and density increased during the next acquisitions performed in June and July. The underlying soil moisture estimation accuracy was on the order of $4 \mathrm{vol} \%$ and $11 \mathrm{vol} \%$ RMSE indicating the potential of the decomposition approach.

\section{INTERFEROMETRY}

SAR interferometry is a powerful and well-established remote sensing technique that enables the highly accurate measurement of important geophysical parameters such as surface topography, ground deformation and subsidence as well as glacier movements [13], [52]-[54]. The key idea of SAR interferometry is to compare for a given scene the phase of two or more complex radar images that have been acquired from slightly different positions or at different times. Since the phase of each SAR image pixel contains range information that is accurate to a small fraction of the radar wavelength, it is possible to detect and measure tiny path length differences with centimetric or even millimetric accuracy. This outstanding accuracy is independent of the distance between the sensor and the scene which makes SAR interferometry highly relevant for both air- and spaceborne remote sensing. Over the last decades numerous terrestrical applications have been demonstrated using either airplanes [18], [55]-[59] or satellites [16], [60]-[70]. A drawback and fundamental challenge of SAR interferometry is, however, that the measured range difference is ambiguous with the wavelength. This ambiguity is typically resolved by using some external information together with appropriate regularity assumptions about the imaged scene, a process known as phase unwrapping [71].

The radar images for SAR interferometry are typically acquired either from mutually displaced flight tracks or from one and the same flight track but at different times. The former is known as across-track interferometry and enables, besides several other applications, a precise measurement of the surface topography. The latter is known as along-track or differential interferometry. By varying the temporal baseline between the interferometric acquisitions, velocities ranging from several meters per second down to a few millimeters per year can accurately be measured. Important applications covering the whole range of potential time scales are the detection of moving objects like cars or ships [70], [72], the observation of ocean surface currents [57], [73], the measurement of sea ice drift and glacier flow [62], [63], [67], the study of seismic deformations

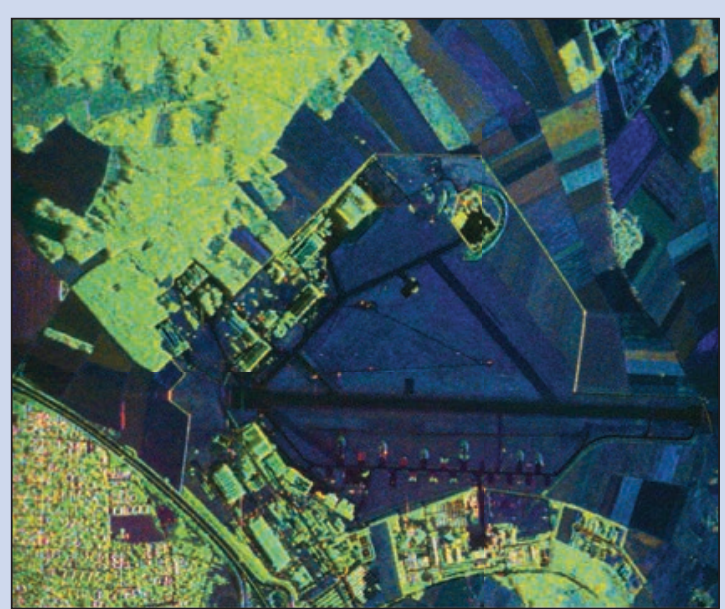

(a)

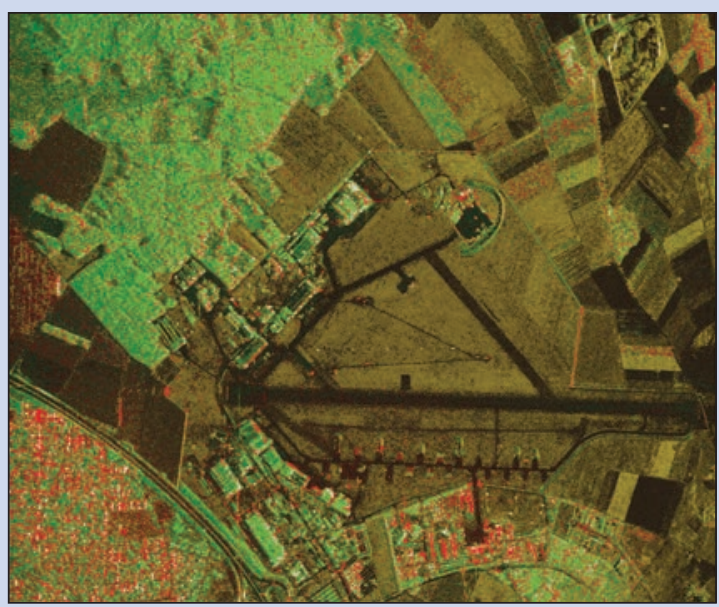

(b)

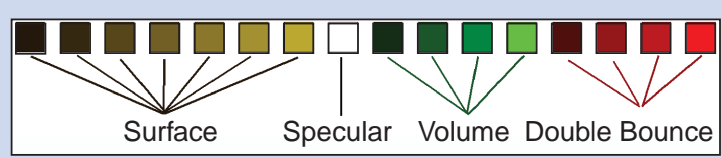

FIGURE 9. (a) Freeman and Durden decomposition applied on quad-polarimetric L-band data acquired over the Oberpfaffenhofen test site. Red, green and blue correspond to $f_{d}, f_{v}$ and $f_{s}$. (b) 16-class Wishart classification initialized by using the three classes derived by the dominant Freeman and Durden $f_{d}, f_{v}$ and $f_{s}$ amplitudes.

and volcanic activities [16], [66], as well as the monitoring of land subsidence [69]. Further potential arises from a comparison of the coherence between several data acquisitions, which can be used for land classification and change detection [64], [74], [75].

\section{A. ACROSS-TRACK INTERFEROMETRY}

Conventional SAR imaging implies a projection from the 3-D object space to a planar 2-D radar image where the image coordinates are range and azimuth. This means that a single image pixel contains the focused radar echoes 
TABLE 6. POSSIBLE RADAR OBSERVABLES ESTIMATED FROM THE SCATTERING MECHANISMS MODEL-BASED DECOMPOSITION AND THEIR RELATION TO THE ENVIRONMENTAL PARAMETERS/APPLICATION EXAMPLES, SEE (22) [45], [46].

\section{SCATTERING MECHANISMS} MODEL-BASED DECOMPOSITION

Surface scattering

Dihedral scattering

Volume scattering and trunk component

\section{APPLICATION EXAMPLES}

Segmentation and classification Soil moisture estimation (under vegetation cover) of the surface Segmentation and classification Soil moisture estimation (under vegetation cover) of the surface

Segmentation and classification Estimation of the volume component (random vs oriented volumes)

displaced from that of the first antenna. As illustrated in Fig. 11, the antenna displacement causes for each scatterer of the imaged scene a characteristic range difference that can be evaluated to retrieve the surface height.

From Fig. 11 it becomes apparent that for large distances $r_{0}$ and short baselines $B_{\perp}$ the one-way range difference $\Delta r$ will be proportional to the height difference $\Delta h$. This proportionality can be expressed as

$$
\Delta r \cong \frac{B_{\perp}}{r_{0} \sin \left(\theta_{i}\right)} \cdot \Delta h,
$$

where $r_{0}$ is the slant range, $\theta_{i}$ is the local incident angle, and $B_{\perp}$ is the baseline perpendicular to the line of sight. In a coherent radar, this range difference $\Delta r$ corresponds to a measured phase difference $\Delta \varphi$

$$
\Delta \varphi=m \frac{2 \pi}{\lambda} \Delta r,
$$

from all elemental scatterers that are contained within a circular ring or torus centered on the platform path. The radius of the ring is given by the range and its length and width are provided by the azimuth and range resolutions, respectively. As a result, a single SAR image does not contain information about the elevation angle and therefore the height of the imaged scene.

The objective of across-track interferometry is to overcome this limitation and to resolve the elevation angle with high precision. For this, the scene is mapped with a second antenna that moves along a flight path which is laterally

where $\lambda$ is the carrier wavelength. The factor $m$ takes into account whether the range difference is only due to the receive path or due to both the transmit and the receive paths. Therefore, $m$ is equal to one for a single-pass SAR interferometer where only one antenna transmits and two antennas receive the scattered echoes, and $m$ is equal to two for a repeat-pass or ping-pong scenario where each radar image is acquired with its own illuminator.

Figure 12 shows as an example the phase differences obtained for a pair of SAR images that were acquired by the spaceborne single-pass SAR interferometer
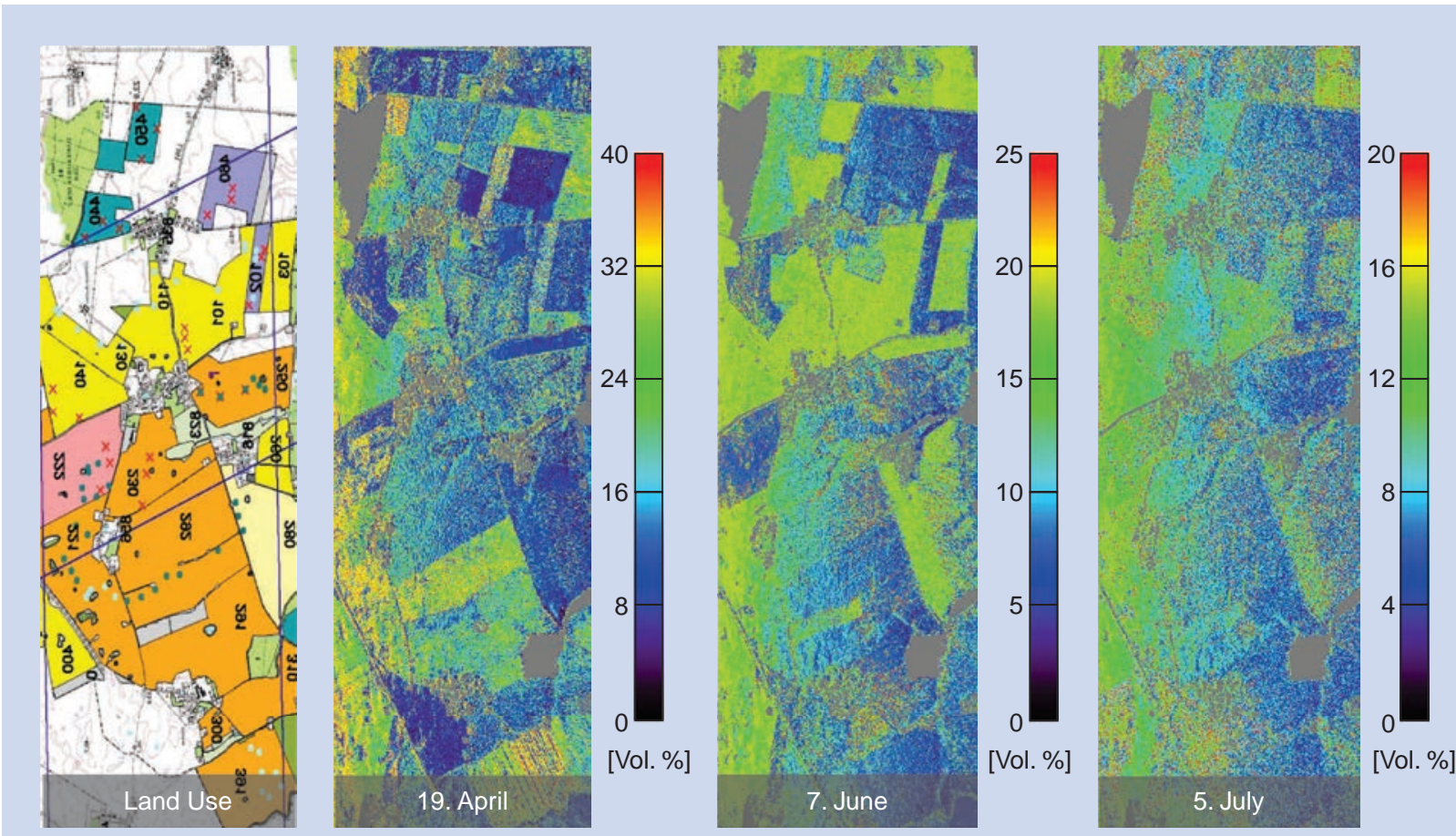

FIGURE 10. Soil moisture maps obtained after applying a model-based polarimetric decomposition to remove the vegetation layer and inverting the remaining ground component. The underlying soil moisture estimation accuracy was on the order of 4 vol\% and 11 vol\% [50]. 
TanDEM-X [76]. To obtain the phase, the two complex SAR images were first aligned such that corresponding image pixels map the same scene features. This alignment is known as co-registration and it is typically based on a maximization of the cross-correlation by applying local range and azimuth shifts between the two SAR images. After this, corresponding image pixels should contain the signals from the same elemental scatterers. After co-registration, the first image was multiplied by the complex conjugate of the second image to form the complex interferogram from which the phase is immediately extracted. This phase is shown in the left image by a cyclic color code as indicated by the color bar. It becomes evident that the interferometric phase is dominated by a fringe pattern in the range direction (range is shown from left to right, and azimuth from top to bottom). This pattern, which is only slightly distorted by the scene topography, is mainly caused by the systematic decrease of the interferometric phase with increasing range and look angle that occurs even for flat surfaces. This flat Earth contribution is typically removed by subtracting the expected phase from an appropriately chosen reference surface and the resulting interferogram is shown in the middle of Fig. 12. Note that the remaining fringes show already some similarity to the contour lines of a topographic map.

The accuracy of the phase measurement in Fig. 12 is limited by the magnitude of the interferometric coherence, which describes the degree of correlation between the two radar images. In practice, several factors contribute to a reduction of the coherence. One major contributor is receiver noise. Assuming identical noise power for both receivers, the corresponding coherence loss is given by [77]

$$
\gamma_{\mathrm{SNR}}=\frac{1}{1+\mathrm{SNR}^{-1}}
$$

where SNR is the signal-to-noise ratio associated with each receiver. Another important factor is temporal decorrelation $\gamma_{\text {Temp, }}$ which describes changes in the scene microstructure and permittivity between two time-separated image acquisitions. Temporal decorrelation may for example be caused by small mutual shifts between the elementary scatterers. Further important decorrelation sources are geometric baseline and volume decorrelation $\gamma_{\mathrm{Geo}}$, which are discussed in later sections. The total coherence loss is then given by a product of the individual losses [78]

$$
\gamma=\gamma_{\mathrm{SNR}} \cdot \gamma_{\mathrm{Temp}} \cdot \gamma_{\mathrm{GeO}} .
$$
repeatedly between 0 and $2 \pi$.

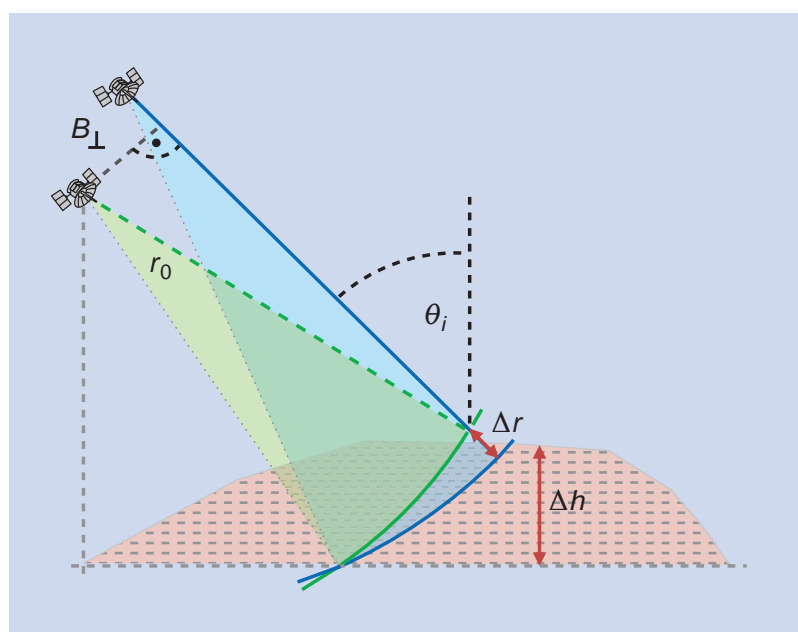

FIGURE 11. Across-track SAR interferometry employs antennas that move on parallel but mutually displaced flight paths (the flight paths are not shown since they are pointing into the page). The slant range $r_{0}$, the incident angle $\theta_{i}$ and the effective baseline $B_{\perp}$ are all defined in a plane perpendicular to the flight paths. A change in the surface height by $\Delta h$ causes a change in the range difference by $\Delta r$.

Coherence loss may cause rather large phase errors in the interferogram. To mitigate phase noise, the complex interferogram is often locally averaged prior to the extraction of the phase values. This averaging is typically denoted as multi-looking and Fig. 13 shows how the phase noise is reduced with an increasing number of averaged interferometric samples (looks). It becomes clear that multi-looking

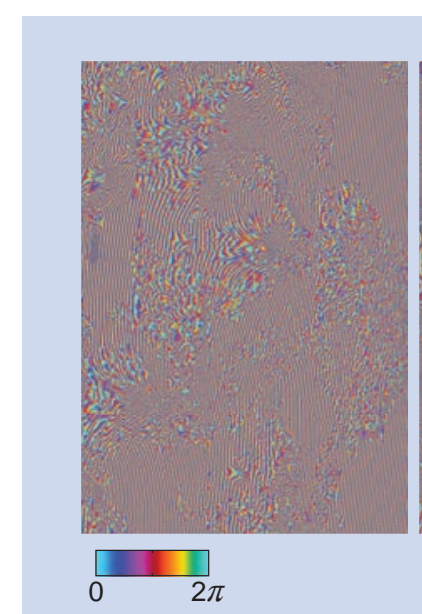

(a)

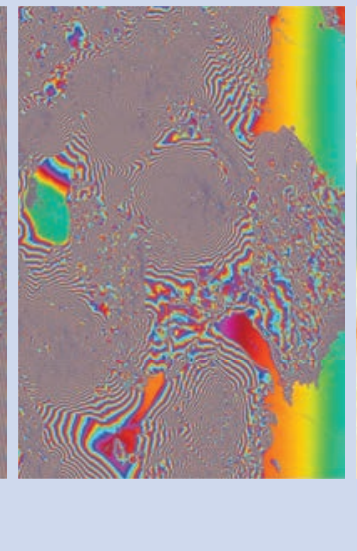

(b)

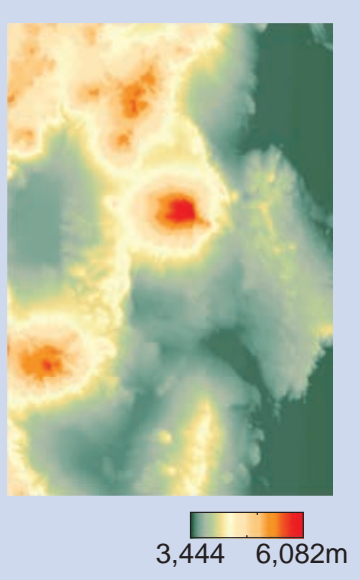

(c)

FIGURE 12. SAR interferograms and digital elevation model (DEM) of the Atacama desert (Chile). The interferometric data have been acquired with the spaceborne single-pass SAR interferometer TanDEM-X [76]. (a) Before removal of phase from flat Earth, where the flat Earth term has been reduced by a factor of four for visualization purposes. (b) After removal of flat Earth phase. (c) Unwrapped phase converted into height values as shown in the color bar below. The color bar on the left indicates the wrapped interferometric phase values which vary 


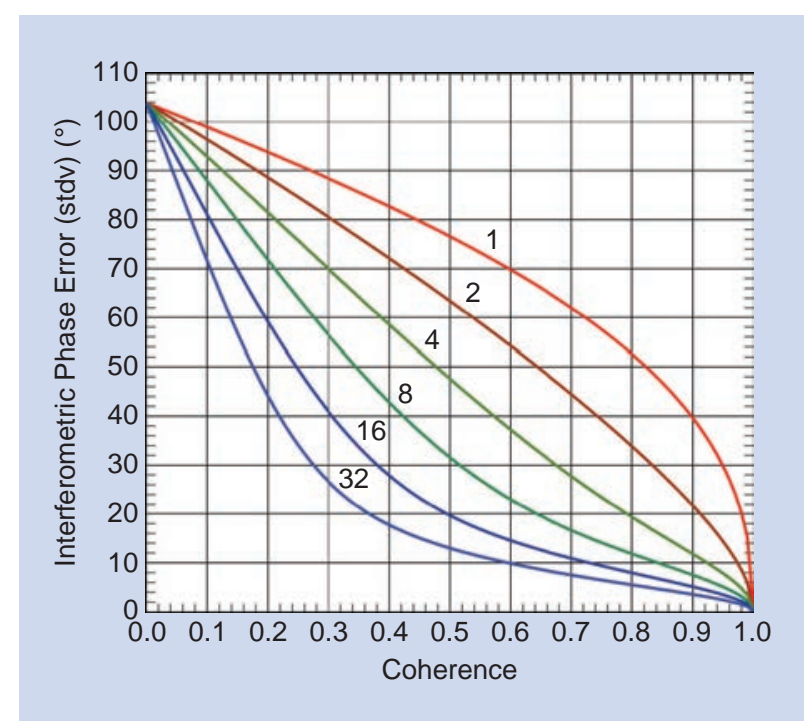

FIGURE 13. Standard deviation of the interferometric phase as a function of the coherence. The six curves illustrate the systematic decrease of the phase noise with increasing look numbers.

SAR INTERFEROMETRY COMPARES THE PHASE

OF TWO COMPLEX RADAR

IMAGES FOR HIGHLY

ACCURATE ELEVATION

MEASUREMENTS. is quite efficient in improving the phase estimates, but it has to be traded against the associated loss of spatial resolution. The interferogram in Fig. 12 was obtained using 5 looks in range and 5 looks in azimuth, which reduced the phase noise standard deviation by approximately a factor of 5 .

To further explore the peculiarities of SAR interferometry, we insert (27) into (28) and obtain after a division by $\Delta h$

$$
\frac{\Delta \varphi}{\Delta h}=\frac{2 m \pi B_{\perp}}{\lambda r_{0} \sin \left(\theta_{i}\right)}
$$

This equation describes the sensitivity of the radar interferometer to small height differences $\Delta h$. It is obvious that the sensitivity can be improved by increasing the length of the perpendicular baseline $B_{\perp}$. However, the maximum useful baseline length is constrained by two factors.

A first limitation is baseline decorrelation. To understand this limitation, we have to remember that the recorded SAR signal can be regarded as being composed of the radar echoes from a large number of closely spaced elementary point-like scatterers with random amplitude and phase. Each of these scatterers contributes to the overall radar signal with an additional phase shift, which is proportional to its distance from the receiving antenna. If we consider now a fixed scatterer ensemble on the ground and vary the radar look angle, it becomes clear that the relative phase between the radar echoes from the individual scatterers changes. The difference will be small for short baselines $B_{\perp}$, but with increasing baseline length $B_{\perp}$ the phase contributions from the elementary scatterers within each resolution cell will become more and more different between the two SAR images. As a result, the correlation between the two complex SAR images decreases systematically with increasing baseline length until it completely vanishes. The baseline length for which the two SAR images become completely decorrelated is known as the criti-

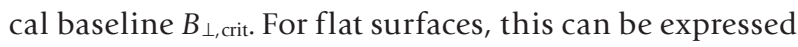
mathematically as [77], [78]

$$
B_{\perp, \text { crit }}=\frac{\lambda r_{0} \tan \left(\theta_{i}\right)}{m \delta_{r}} .
$$

For baselines that are smaller than the critical baseline, the spatial surface decorrelation can be removed by a process known as range filtering at the cost of a degraded range resolution [79]. Note that the length of the critical baseline increases with decreasing range resolution. Modern SAR systems have typically a rather large bandwidth and baseline decorrelation is therefore nowadays a less important issue than it was with early SAR systems.

A second and from a practical point of view often more restrictive limitation for the maximum useful baseline length results from ambiguities in the phase-to-height conversion process. For this we consider again (31) and recall that the interferometric measurement provides only phase values which are ambiguous by integer multiples of $2 \pi$. As a result, the height measurements are also ambiguous by multiples of

$$
h_{\mathrm{amb}}=\frac{\lambda m r_{0} \sin \left(\theta_{i}\right)}{B_{\perp}} .
$$

Such ambiguities are usually resolved during phase unwrapping, which exploits spatial correlations between the height values arising from natural topography [13], [52], [60], [71]. The right-hand side of Fig. 12 shows the unwrapped phase of the Atacama interferogram, which is scaled to terrain height in accordance with the factor given in (31). The accuracy of this phase (or height) reconstruction process depends on several factors like the signal-to-noise ratio, the surface and volume decorrelation (cf. Section V-A), the ground resolution, and, most important, the actual terrain itself. The latter may strongly limit the useful baseline length for rough terrain like deep valleys, isolated peaks, tall forests, or mountains with steep slopes. On the other hand, large baselines are desired to achieve a sensitive radar interferometer with a good phase-to-height scaling. This dilemma becomes especially pronounced for state-of-the-art radar sensors, which will provide a high range bandwidth and hence enable coherent data acquisitions with long interferometric baselines. To illustrate this problem, we consider a spaceborne radar interferometer like TanDEM-X [76], which operates in the interferometric imaging mode 
with a nominal bandwidth of $B_{r}=100 \mathrm{MHz}$. Choosing a baseline which is just $10 \%$ of the critical baseline, the corresponding height of ambiguity is about $10 \mathrm{~m}$ for an incident angle of $\theta_{i}=45^{\circ}$. Such a height of ambiguity is quite low and it may cause irresolvable height errors in areas with rough terrain. It is therefore in general not possible to take full advantage of the opportunity for large baseline acquisitions provided by the new generation of high-bandwidth radar systems.

A possible solution to this dilemma is an interferometric system with flexible baseline lengths. This enables an adaptation of the baseline to the actual terrain and it offers furthermore the possibility to image one and the same area with multiple baselines of different length. The latter can be used for an unambiguous reconstruction of the terrain height [80], [81]. Such a strategy is also employed in the TanDEM-X mission where two or more interferograms with a typical baseline ratio of 0.7 are combined to resolve height ambiguities. The results obtained with this technique are rather promising [82]. In the future, one may also use systems that allow for the acquisition of multiple baselines in a single-pass. An example are satellite formations with multiple spacecraft that allow for adjustable baselines ranging from less than 100 meters up to 10 kilometers and more [83]. The interferogram in Fig. 12 was obtained with a height of ambiguity of $47 \mathrm{~m}$ and the terrain allowed for successful phase unwrapping without the need for a second acquisition with a different baseline.

The last steps in the DEM generation process are phase-to-height conversion and geocoding. The phase-toheight conversion uses the imaging geometry of Fig. 11 in combination with the interferometrically derived range difference to determine by trigonometry for each image point its position relative to the sensor. This requires both a sufficiently accurate knowledge of the interferometric baseline between the radar antennas and a precise knowledge of the range difference for each scatterer. Since the latter is derived from the unwrapped interferometric phase, it is unfortunately only known up to a global offset which is a multiple of the wavelength. The remaining uncertainty is typically resolved by using at least one external reference. An alternative is the employment of radargrammetric techniques, which measure the mutual range shift between corresponding SAR image pixels to derive a coarse DEM. The radargrammetric measurement is unambiguous but its accuracy is typically two orders of magnitude worse than that of the interferometric technique. Nevertheless, a sufficient accuracy can be obtained by averaging over a large scene as it is done in the operational TanDEM-X processor [84]. The final step is geocoding, which involves a transformation from the radar geometry to the coordinates of a selected geodetic reference system. Fig. 14 shows as an example the geocoded digital elevation model (DEM) that has been derived by the experimental TAXI processor [85] from the Atacama interferogram of Fig. 12.

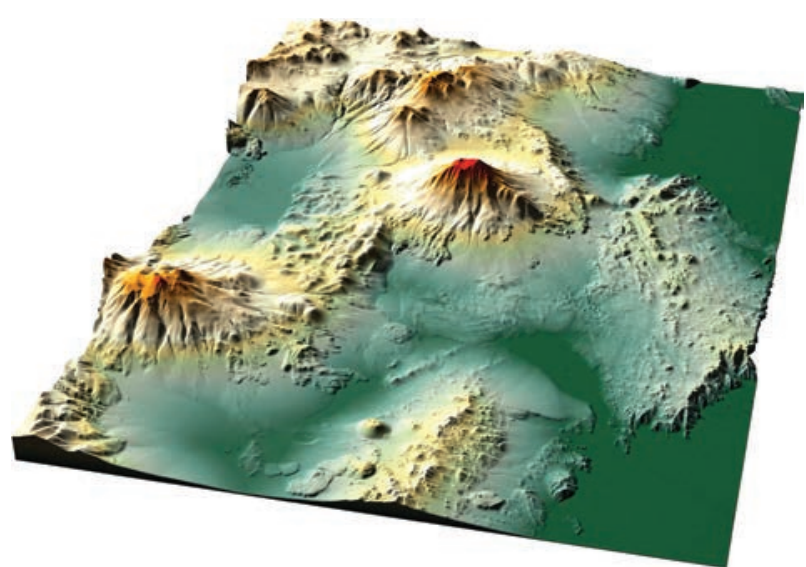

FIGURE 14. Geocoded digital elevation model (DEM) derived from the unwrapped interferometric phase of Fig. 12.

\section{B. DIFFERENTIAL SAR INTERFEROMETRY}

Differential SAR interferometry (DInSAR) is a further clear example of a well-established interferometric technique. Similar as with InSAR, the high sensitivity of a SAR instrument to measure the LOS propagation distance is exploited in order to detect displacements of the Earth surface at a wavelength scale.

Consider two SAR images acquired with a certain temporal separation that are combined to generate an interferogram. Ideally, a zero-baseline configuration would result in an interferogram whose phase information would only be related to the LOS displacement in the scene. In practice though, a certain baseline is always present, which makes the interferogram also sensitive to the topography of the scene. By using an external DEM, the topographic information can be subtracted from the interferogram, leading to a differential SAR interferometric measurement where subtle changes of the range distance between the two acquisitions (e.g., due to subsidence) can be detected. After the topographic phase removal, the phase of the interferogram becomes

$$
\varphi_{\text {disp }}=\frac{4 \pi}{\lambda} \Delta r_{\text {disp }}
$$

where $\Delta r_{\text {disp }}$ is the desired LOS displacement. As it can be noted in (34), DInSAR can achieve an accuracy in the order of a fraction of the wavelength in the measurement of the LOS displacement, hence becoming a powerful tool for deformation monitoring of large areas. Similar to InSAR, the differential phase needs to be unwrapped and calibrated in order to obtain absolute displacement results (see example in Fig. 15(a)). But several aspects impair the performance of this approach. First, the accuracy of the external DEM needs to be in the same order of magnitude or better than the phase sensitivity of the interferometric baseline. But more important, one needs to consider phase noise due to temporal decorrelation and variations in the propagation medium. The latter is a consequence of the atmospheric delay, which is mainly due to the water 


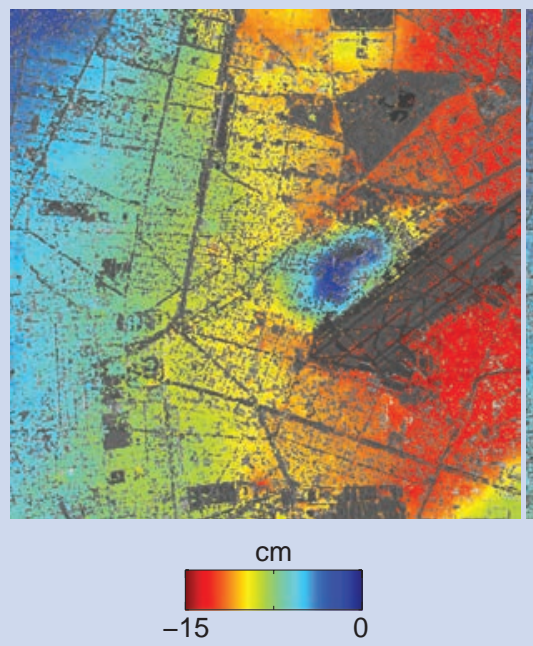

(a)
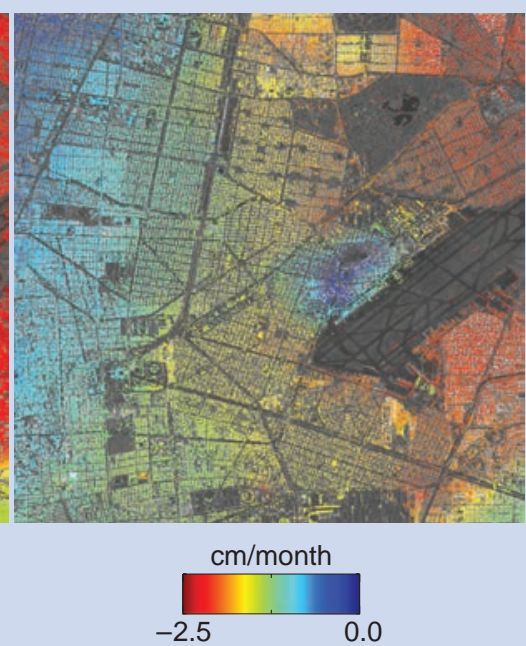

(b)

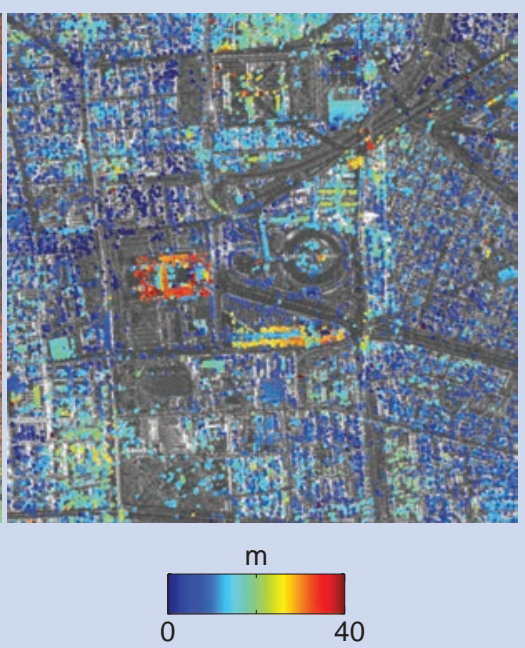

(c)

FIGURE 15. (a) Estimated subsidence over Mexico City obtained with two TerraSAR-X images acquired with a 6-month difference (overlay of reflectivity and phase). Low coherence areas have been masked out. (b) Mean deformation velocity estimated over Mexico City using the PS technique. (c) Zoom over the city of the refined DEM retrieved as an additional product to the deformation velocity, where the individual buildings can be observed. The size of the PSs has been enlarged for visualization purposes. The scene size is approximately $8 \mathrm{~km} \times 8 \mathrm{~km}$. Radar illumination from the right.

vapor content in the troposphere. The delay is in the order of 2-4 meters, but its gradient at a local scale can be in the order of up to $1 \mathrm{~cm} / \mathrm{km}$ or more [54], [86], hence limiting the accuracy of the conventional differential SAR interferometry approach, and being only of interest for cases where the displacements are larger than this value. At lower frequency bands like

DIFFERENTIAL SAR

INTERFEROMETRY CAN

DETECT DISPLACEMENTS

OF THE EARTH SURFACE AT A

SUBWAVELENGTH SCALE.
L- or P-band, the total electron content (TEC) inside the ionosphere results in a further non-negligible path delay, which can potentially vary within the synthetic aperture time, and thus introduce undesired defocusing and impulse response shifts in the azimuth dimension [87], [88].

Figure 15(a) shows the subsidence over Mexico City estimated with two TerraSAR-X images acquired with a 6-month difference, where the SRTM DEM [89] was used to remove the topographic phase. The maximum displacement is about $15 \mathrm{~cm}$ in some city areas, corresponding roughly to a factor 5 of the wavelength. The areas with a low coherence have been masked out for visualization purposes. The subsidence is due to ground water extraction and it is a well-known problem in Mexico City [90].

The exploitation of image time series is the solution to the limitations of conventional differential SAR interferometry. By using a large stack of images acquired at different time instants, the aforementioned contributions can be separated. The signal model of a single point in the image stack for a given interferometric pair can be written as

$$
\varphi_{i, j}=\varphi_{\mathrm{topo}}+\varphi_{\mathrm{disp}}+\varphi_{\mathrm{atm}}+\varphi_{n,}
$$

where $\varphi_{\text {topo }}$ represents the residual topographic component after removing the external DEM, $\varphi_{\text {atm }}$ the atmospheric component, $\varphi_{n}$ the phase noise, and $i$ and $j$ represent the image indexes within the stack. But the main challenge is, similar as with InSAR, the fact that the phases are wrapped modulo $2 \pi$, which makes the problem non-linear.

Several approaches have been developed in the literature in order to extract the different contributions of interest through different advanced filtering schemes. The first step in these approaches is the selection of the image pixels with enough quality to be used in the estimation process, being the most commonly approaches the ones based on the amplitude dispersion, i.e., the socalled permanent scatterers (PS) technique [17], [69], or on the interferometric coherence [91]-[93]. A PS appears in the image as a pixel showing a stable amplitude behavior in time and usually corresponds to point-like targets such as buildings or rocks, while distributed scatterers are discarded. On the other hand, the selection using the coherence further includes distributed scatterers that remain coherent in time, e.g., surfaces, hence increasing the potential number of candidate pixels. Nevertheless, the coherence estimation implies a resolution loss in opposition to the PS technique, which works at full 
image resolution. Recent approaches exploit both PS and coherent distributed scatterers simultaneously in order to increase the number of valid pixels, and hence achieve a larger coverage of the measurement [94]. The selection of valid pixels is, in any case, an intense topic of research, where the objective is to find as many as possible valid pixels at the best spatial resolution [39], [95]-[100].

The non-linearity of the problem, i.e., the wrapping of the phases, is also addressed differently. Some techniques perform the phase unwrapping of the individual interferograms on a sparse grid to turn the problem linear and ease the forthcoming filtering steps [69], [91], while others exploit phase differences between neighboring pixels working in the complex domain (non-linear optimization), being nevertheless required to perform an integration process afterwards [92], [97]. More advanced approaches perform 3-D phase unwrapping, i.e., including the time variable, hence gaining some more robustness in comparison to the previous approaches [101]-[103].

Once the pixels have been selected, a first estimation of $\varphi_{\text {topo }}$ and a given deformation model, usually linear, is performed simultaneously. After removing these two components from the image stack, a new set of residual differential interferograms is obtained, which mainly contain the residual motion of the scatterer, the atmospheric phase screen (APS), and phase noise. By exploiting the frequency response of the APS both in time (high-pass) and space (low-pass), it can be estimated and removed from the image stack [17], [69]. Finally, the residual motion remains, which added to the previously estimated deformation, delivers the temporal evolution of the deformation for each pixel.

Figure 15(b) shows an example of the estimated mean deformation velocity over Mexico City using the PS technique with an image stack of 36 TerraSAR-X stripmap images. As an additional product, an accurate DEM is obtained (see Fig. 15(c)). With current high-resolution spaceborne sensors like TerraSAR-X or COSMO-SkyMed, the topography retrieval turns into an interesting alternative to Lidar for accurate DEM generation of cities in a global scale [104]-[106]. Furthermore, the estimated APS can be also exploited for atmospheric modeling and water vapor retrieval [54], [86].

\section{POLARIMETRIC SAR INTERFEROMETRY}

Polarimetric SAR Interferometry (Pol-InSAR) is based on the coherent combination of single- or multi-baseline interferograms acquired at different polarizations [18], [35], [107]. Combining the potential of radar polarimetry to identify individual scattering processes and to amplify or attenuate them with respect to each other with the sensitivity of SAR interferometry to the spatial variability of vertical structure parameters allows to build up a unique observation space for the characterization of the vertical distribution of scattering processes in natural and artificial volume scatterers.

\section{A. INTERFEROMETRIC COHERENCE: INFORMATION CONTENT}

The key observable in Pol-InSAR measurements is the complex interferometric coherence (that includes both, the interferometric correlation coefficient and interferometric phase) measured at different polarizations. As discussed in Section IV-A (see (30)), $\gamma$ depends on instrument and acquisition parameters as well as on dielectric and structural parameters of the scatterer [52], [77], [108]. The geometric baseline decorrelation term $\gamma_{\mathrm{Geo}}$ expresses the angular dependency of the scattering reflectivity in terms of amplitude and phase.

Due to the slightly different incidence angles induced by the spatial baseline, the projection of the 3-D scatterer into the two interferometric SAR images is different. Accordingly, $\gamma_{\text {Geo }}$ expresses the (horizontal and vertical) spectral correlation properties of the scatterers reflectivity [52]. Assuming the scatterers reflectivity to be locally invariant in the horizontal direction, $\gamma_{\text {Geo }}$ can be further decomposed into

$$
\gamma_{\mathrm{GeO}}=\gamma_{\mathrm{Az}} \gamma_{\mathrm{Rg}} \gamma_{\mathrm{Vol}}
$$

Range and azimuth spectral decorrelation $\gamma_{\mathrm{Rg}}$ and $\gamma_{\mathrm{Az}}$ are caused by the different projection of the scatterer groundrange and azimuth spectrum into the two images forming the interferogram. The difference in range, as explained in section IV-A is due to the slightly different incidence angles while the difference in azimuth is due to different Doppler centroids. In the case of distributed scatterers the non-common spectral parts decorrelate when forming the interferogram. Both contributions can be compensated (i.e., $\gamma_{\mathrm{Rg}}=1$ and $\gamma_{\mathrm{Az}}=1$ ) by the so-called common spectral band filtering, i.e., by filtering the range and azimuth bandwidth of the two images at different central frequencies excluding the non-common parts [52], [79].

Volume decorrelation $\gamma_{\mathrm{Vol}}$ is the decorrelation caused by the different projection of the vertical component of the scatterer reflectivity spectrum into the two interferometric SAR images. It contains therefore information about the vertical structure of the scatterer [52], [108]. Indeed, $\gamma_{\mathrm{Vol}}$ is directly related to the vertical distribution of scatterers $F(z)$ in the medium through a (normalized) Fourier transformation relationship [35], [52]

$$
\gamma_{\mathrm{Vol}}=\exp \left(\mathrm{i} k_{z} z_{0}\right) \frac{\int_{0}^{h_{v}} F\left(z^{\prime}\right) \exp \left(\mathrm{i} k_{z} z^{\prime}\right) \mathrm{d} z^{\prime}}{\int_{0}^{h_{v}} F\left(z^{\prime}\right) \mathrm{d} z^{\prime}},
$$




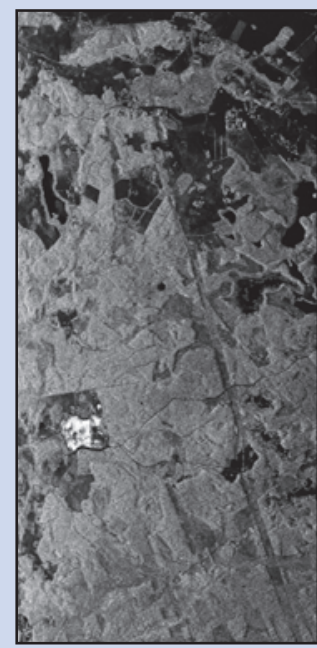

(a)

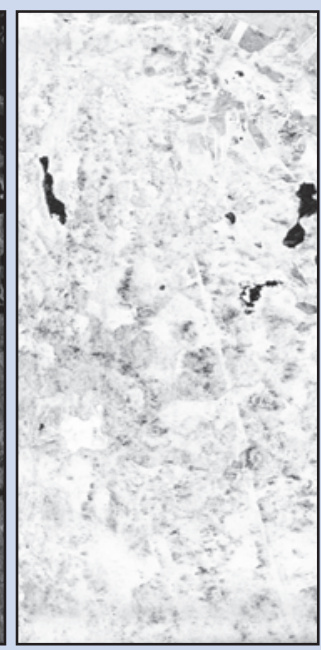

(b)

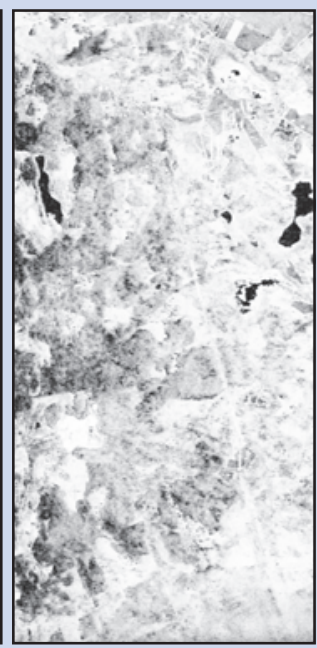

(c)

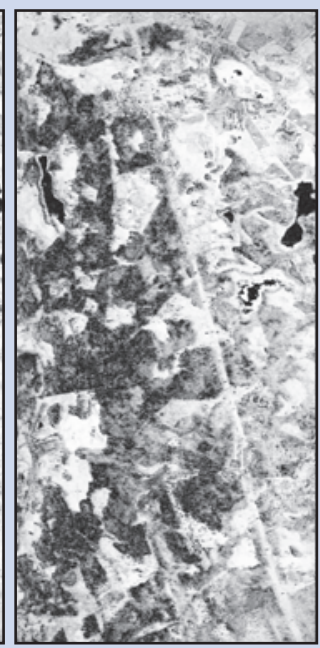

(d)

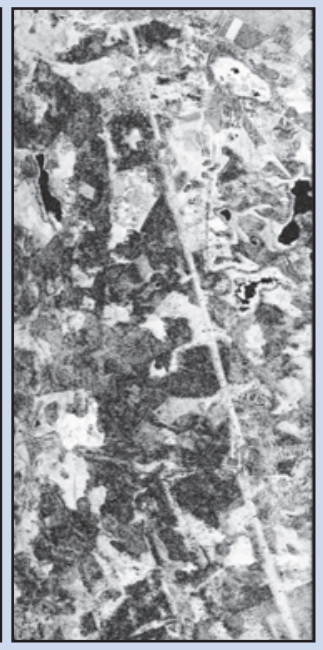

(e)

FIGURE 16. L-band amplitude image (HV polarization) of the Remingstorp test site (a). Four coherence images (scaled from 0 (black) to one (white)) corresponding to interferograms acquired at different spatial baselines with $k_{z}=0.01,0.05,0.1$ and $0.15 \mathrm{rad} / \mathrm{m}$, respectively (b)-(e).

where $h_{V}$ indicates the height (or depth) of the volume. $k_{z}$ is given by (see (33))

$$
k_{z}=\frac{2 m \pi \Delta \theta}{\lambda \sin \left(\theta_{i}\right)}=\frac{2 \pi}{h_{\mathrm{amb}}}
$$

and corresponds to the effective vertical (interferometric) wavenumber that depends on the imaging geometry $(\Delta \theta$ is the incidence angle difference between the two interferometric images induced by the baseline and $\theta_{i}$ the local incidence angle) and the radar wavelength $\lambda . z_{0}$ is a reference height and $\varphi_{0}=k_{z} z_{0}$ the associated interferometric phase.

Figure 16 illustrates the dependency of $\gamma_{\mathrm{Vol}}$ on the vertical structure dependency $F(z)$ : On the left-hand side is the L-band amplitude image (HV polarization) of the Remingstorp test site located in Sweden. It is a forested site and includes a number of bare fields and lakes. The four coherence images shown on the right (scaled from 0 (black) to one (white)) correspond to interferograms acquired at different spatial baselines with $k_{z}=0.01,0.05,0.1$ and $0.15 \mathrm{rad} / \mathrm{m}$, respectively. The temporal baseline for each interferogram is about 5 minutes so that temporal decorrelation can be - at least in first order - neglected. As expected from (37), bare fields, characterized by a Dirac-like vertical scattering contribution have, after range and azimuth common-band filtering, a coherence

$$
\gamma=\gamma_{\mathrm{SNR}} \gamma_{\mathrm{Vol}}=\gamma_{\mathrm{SNR}} \exp \left(\mathrm{i} k_{z} z_{0}\right),
$$

whose magnitude $|\gamma|=\gamma_{\mathrm{SNR}}$ is independent of the spatial baseline (i.e., $k_{z}$ ). The loss of coherence is due to SNR decorrelation. In contrast to them, forested stands are characterized by an extended vertical scattering contribution that leads to a monotonically declining $\gamma_{\mathrm{Vol}}\left(k_{z}\right)$ behavior.
Indeed, $\gamma=\gamma_{\mathrm{SNR}} \gamma_{\mathrm{Vol}}$ is decreasing over all forested areas in the image. Note that the lakes decorrelate at each baseline primarily due to SNR decorrelation.

Accordingly, $\gamma_{\text {Vol }}$ contains the information about the vertical structure of the scatterer and is the key observable for the quantitative estimation of volume parameters from Pol-InSAR measurements.

\section{B. INFORMATION EXTRACTION FROM InSAR COHERENCE INVERSION}

The estimation of $F(z)$ (and/or associated structure parameters) from $\gamma_{\mathrm{Vol}}\left(k_{z}, \vec{w}\right)$ measurements at different polarizations and (spatial) baselines according to (37) is a unique opportunity provided by Pol-InSAR observations. Indeed, for the estimation of $F(z)$ (and/or associated structure parameters) from $\gamma_{\mathrm{Vol}}\left(k_{z}, \vec{w}\right)$ measurements at different polarizations (where the unitary vector $\vec{w}$ indicates the polarization dependency [18], [107] and (spatial) baselines by means of (37) two approaches have been explored in the literature:

1) The first one is to model $F(z)$ by parameterizing its shape in terms of geometrical and scattering properties and to use then $\gamma_{\mathrm{Vol}}\left(k_{z}, \vec{w}\right)$ measurements to estimate the individual model parameters. In this case, the scattering model is essential for the accuracy of the estimated parameters. On the one hand, the model must contain enough physical structure to interpret the Pol-InSAR coherences, while, on the other hand, it must be simple enough in terms of parameters in order to be determinable with a limited number of observations.

For natural volume scatterers multilayer statistical models that account for the different scattering and propagation properties at the different heights (depths) within 
the volume by using macroscopic statistical and/or symmetry derived scattering properties have been established due to their flexibility and the (relative) small number of parameters needed for parameterization. For vegetation applications two layer statistical models, consisting of a vertical distribution of scatterers $f(z)$ that accounts for the vegetation scattering contribution, and a Dirac-like component $m_{\mathrm{G}}(\vec{w}) \delta\left(z-z_{0}\right)$ that accounts for the scattering contribution(s) with the underlying ground (i.e., direct surface and dihedral vegetation-surface contributions) have been proven to be sufficient in terms of robustness and performance especially at lower frequencies [18], [35], [109], [110]

$$
F(z, \vec{w})=f(z, \vec{w})+m_{\mathrm{G}}(\vec{w}) \delta\left(z-z_{0}\right)
$$

where $m_{\mathrm{G}}$ is the ground scattering amplitude. Substituting (40) into (37) leads to

$$
\gamma_{\mathrm{Vol}}\left(k_{z}, \vec{w}\right)=\exp \left(\mathrm{i} \varphi_{0}\right) \frac{\tilde{\gamma}_{V 0}+m(\vec{w})}{1+m(\vec{w})}
$$

where

$$
\tilde{\gamma}_{V 0}=\frac{\int_{0}^{h_{V}} f_{V}\left(z^{\prime}\right) \exp \left(\mathrm{i} k_{z} z^{\prime}\right) \mathrm{d} z^{\prime}}{\int_{0}^{h_{V}} f_{V}\left(z^{\prime}\right) \mathrm{d} z},
$$

and

$$
m(\vec{w})=\frac{m_{\mathrm{G}}(\vec{w})}{\int_{0}^{h_{V}} f_{V}\left(z^{\prime}\right) \mathrm{d} z^{\prime}}
$$

is the effective ground-to-volume amplitude ratio.

For modeling the vertical distribution of scatterers in the vegetation layer $f(z)$ different models can be used. A widely and very successfully used model for $f(z)$ is an exponential distribution of scatterers [18], [109], [111]

$$
f_{V}(z)=\exp \left(2 \sigma z / \cos \left(\theta_{i}\right)\right)
$$

where $\sigma$ is a mean extinction value for the vegetation layer that defines the "attenuation rate" of the profile. Besides the exponential profile, that appears to fit better higher frequencies, Gaussian [112], [113], [114], or linear scattering distributions have been proposed especially at lower frequencies.

Equally important with the shape of the vertical distribution of scatterers $F(z, \vec{w})$ is its polarimetric characterization. While the ground scattering component is strongly polarized and therefore has to be assumed to be polarization dependent, the volume scattering component can be both. In the case of oriented volumes, the vertical distribution of scatterers in the volume is polarization dependent, while in the case of random volumes, the vertical distribution of scatterers in the volume is the same for all polarizations. In forest applications random volumes have been established so that a single polarimetric baseline allows the inversion of the Random-Volume over Ground (RVoG) model [18], [110]. Oriented volumes are more expected to be important in agriculture applications where the scatterers within the agricultural vegetation layer are in many cases characterized by an orientation correlation introducing anisotropic propagation effects and differential extinction [115]-[118].

2) The second approach to estimate $F(z)$ or associated structure parameters is to approximate $F(z)$ by a (normalized) polynomial series or another orthogonal function basis $P_{n}(z)$ [35], [119], [120]

$$
F(z, \vec{w})=\sum_{n} a_{n}(\vec{w}) P_{n}(z)
$$

and to use then $\gamma_{\mathrm{Vol}}\left(k_{z}, \vec{w}\right)$ measurements to estimate the coefficients $a_{n}$ of the individual components. The advantage of this approach is that there is no assumption on the shape of $F(z)$ required, allowing the reconstruction of arbitrary vertical scattering distributions. In its original proposed form [119], [120], $F(z)$ was expressed in terms of the Legendre polynomials $P_{L n}$ leading to

$$
\gamma_{\mathrm{Vol}}\left(k_{z}, \vec{w}\right)=\frac{\exp \left(\mathrm{i} k_{z} z_{0}\right) \exp \left(\mathrm{i} k_{V}\right)}{\int_{-1}^{1}\left[1+\sum_{n} a_{n}(\vec{w}) P_{\text {Ln }}\left(z_{N}\right) \exp \left(\mathrm{i} k_{V}\right)\right] \mathrm{d} z_{N}}
$$

with $k_{V}=k_{z} h_{V} / 2$, where the height of the volume $h_{V}$ and the interferometric phase related to the ground need to be estimated a priori, e.g., using the first approach. According to (46), the availability of multiple Pol-InSAR interferograms makes it possible to determine, in addition to forest height and the underlying ground topography, the vertical distribution of scatterers within the volume.

The Role of Frequency: With changing frequency, the attenuation in the volume changes while at the same time the effective scatterers in the volume - sized on the order of the wavelength - may change, too. Both effects make $F(z)$ (and in consequence the interferometric volume coherence) to vary with frequency [121], [122]. In addition, the maximum volume height/depth that can be assessed is limited by the penetration depth that decreases with increasing attenuation, i.e., frequency.

In the case of vegetation, with decreasing frequency the volume attenuation (and scattering contribution) decreases while the direct and dihedral contribution of the underlying ground increases. The significance of smaller scatterers (as leaves and branches) decreases and larger scatterers are pronounced: As larger scatterers are usually located at the lower vegetation parts, moving to lower frequencies (P-band) may lead to an inversion of the shape of $F(z)$ [122]. 


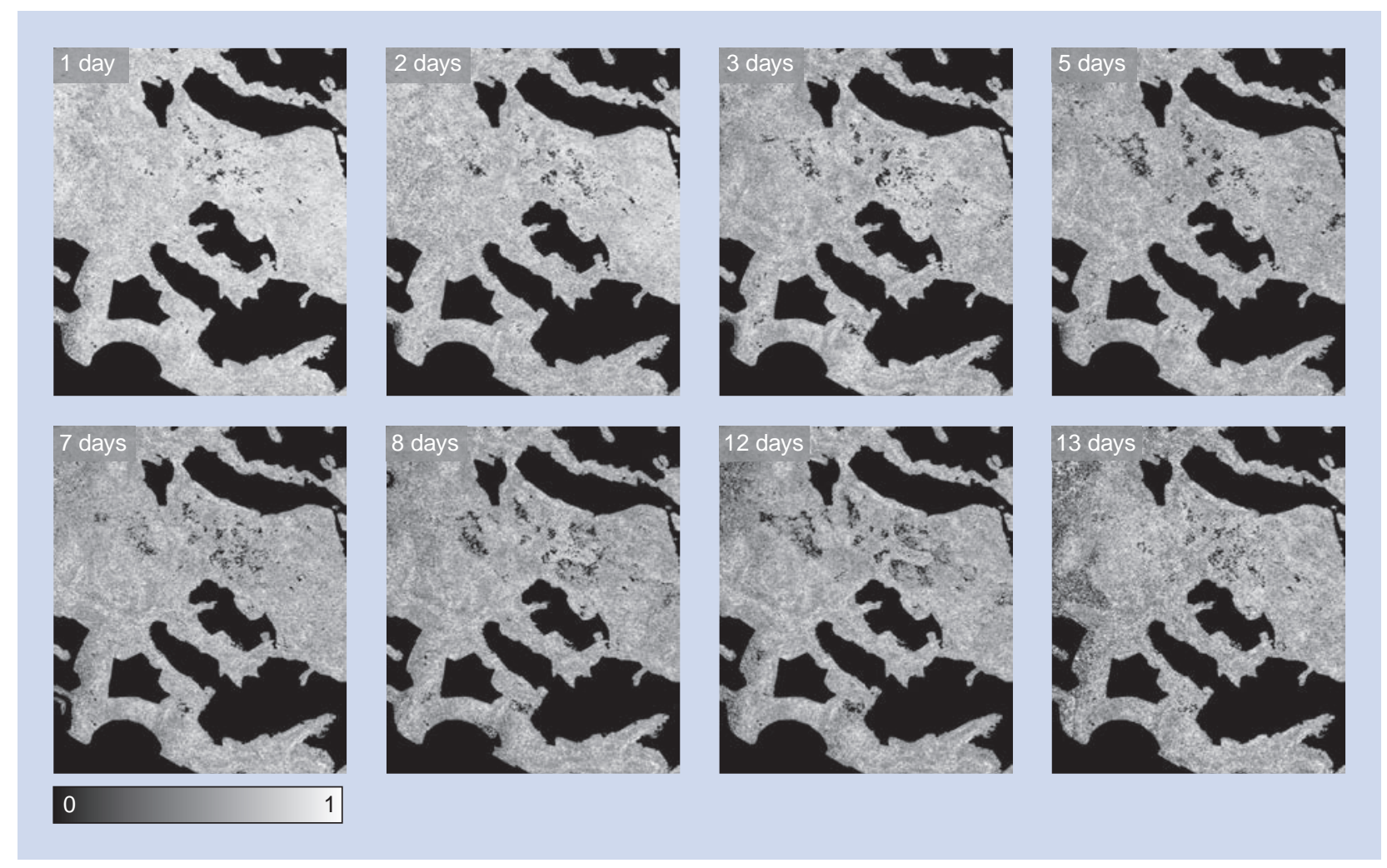

FIGURE 17. Temporal decorrelation maps estimated using repeat-pass interferometry at L-band over the Traunstein test site for different temporal baselines ranging from 1 up to 13 days.

With increasing frequency, volume scattering increases while the underlying ground contribution becomes more and more attenuated. The maximum vegetation height that can be resolved is given by the penetration depth at the given frequency; with further increasing height the interferometer does not "see" anymore the whole volume and the height estimation "saturates."

Temporal Decorrelation: An important step in reaching the optimum Pol-InSAR estimation performance and a critical element in PolInSAR mission design and performance analysis is the calibration of non-volumetric decorrelation contributions [123]-[125]. The most prominent of them is temporal decorrelation in the case of a repeat-pass acquisition scenario. The estimation of temporal decorrelation and its dependency on environmental parameters and temporal baseline at different wavelengths has been the objective of a series of experiments performed in the last years [123]-[126]. Fig. 17 shows the resulting temporal decorrelation maps over the Traunstein test site for a set of temporal baselines at L-band. This makes clear that even very short temporal baselines can be significantly affected by temporal decorrelation. Accordingly, to avoid any temporal decorrelation contributions a single-pass Pol-InSAR configuration is recommended for any operational mission implementation, as in the case of acrosstrack interferometry.

\section{POL-InSAR APPLICATIONS}

Pol-InSAR is today an established technique that promises a break-through in solving essential radar remote sensing problems. Indeed, structural parameters of volume scatterers in the biosphere and cryosphere such as vegetation height, structure, biomass, snow depth, and ice layering are today critical inputs for ecological process modeling and enable monitoring and understanding of eco-system change.

Across the different application fields forest parameter retrieval is by far the most developed one. Forest height estimation from polarimetric single- and multi-baseline data has been demonstrated in a series of airborne experiments over a variety of natural and managed temperate, boreal, and tropical test sites characterized by different stand and terrain conditions [127]-[131]. A single baseline quad-pol acquisition is sufficient for the inversion of (37) and the estimation of forest height. However, the coherent (or even incoherent) combination of multiple Pol-InSAR acquisitions at different spatial baselines allows to obtain 
optimum inversion performance across a wide(r) range of forest heights and a better stability against non-volumetric decorrelation contributions [132].

An example of Pol-InSAR forest height estimation is shown in Fig. 18. In Figure 18(a), an L-band SAR image of the Traunstein forest site, located in southern Germany is shown. The Traunstein forest is characterized by a large variety of forest stand conditions in the presence of locally variable topography. In the middle and on the right of Fig. 18 forest height maps derived from Pol-InSAR data acquired at L-band in 2003 (b) and 2008 (c) are shown. Comparing the two forest height maps a number of changes within the forest become visible: The logging of

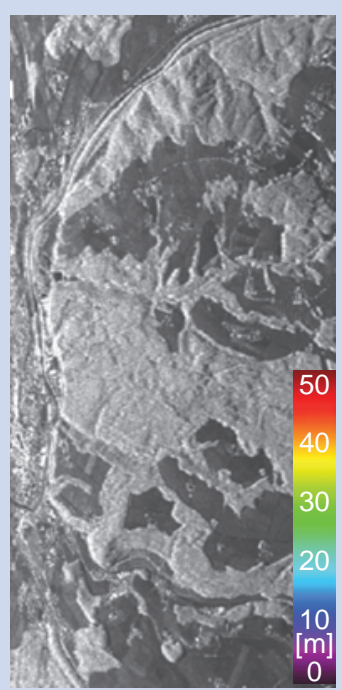

(a)

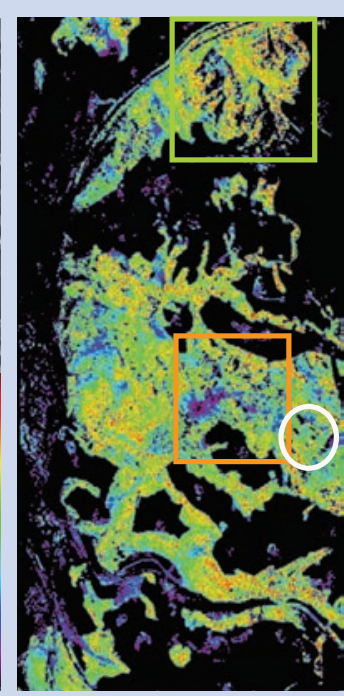

(b)

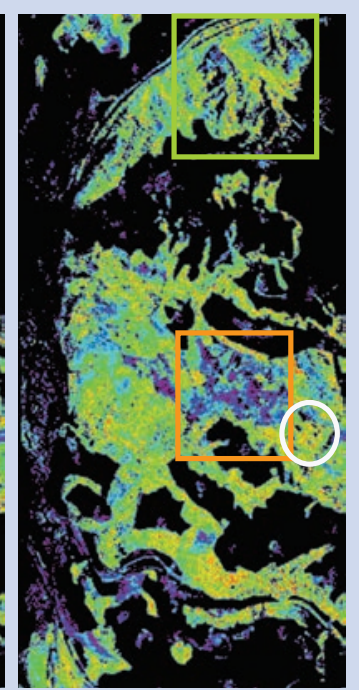

(c)

FIGURE 18. (a) L-band HV intensity image of the Traunstein test site. Forest height map computed from Pol-InSAR data in (b) 2003 and (c) 2008.

a change in forest management between 2003 and 2008 (marked by the green box); the damage caused in January 2007 by the hurricane Kyrill which blew down large parts of the forest (marked by the orange box); and finally forest growth on the order of 3 to $5 \mathrm{~m}$ over young stands as seen within the area marked by the white circle.

The availability of multiple Pol-InSAR interferograms at lower frequencies makes it possible to determine not only forest height and ground topography [133]-[135], but also the lower frequency components of the vertical scattering structure according to (46). Fig. 19 shows two such L-band profiles across the Traunstein test site derived using up to third-order Legendre polynomials by means of (46). The coefficients have been derived using the cross-polarized coherence measured at only two spatial baselines while the ground topography and forest height are both estimated from the inversion of Pol-InSAR interferograms. The structure information allows to distinguish clearly between the younger mixed forest stands characterized by a rather heterogeneous vertical structure distribution from mature spruce stands with their more homogeneous structure profiles defined by their elevated canopies.

Forest height and vertical forest structure are essential for the development of accurate and robust allometric biomass estimators. The initial proposed forest height-to-biomass allometry, even if robust and unsaturated, depends critically on forest stand density. This reduces the estimation accuracy in heterogeneous forest conditions [136], [137]. A way forward is the establishment of a more general allometric relationship between forest biomass and vertical forest structure. This generalized allometry may allow robust and accurate biomass estimation independent

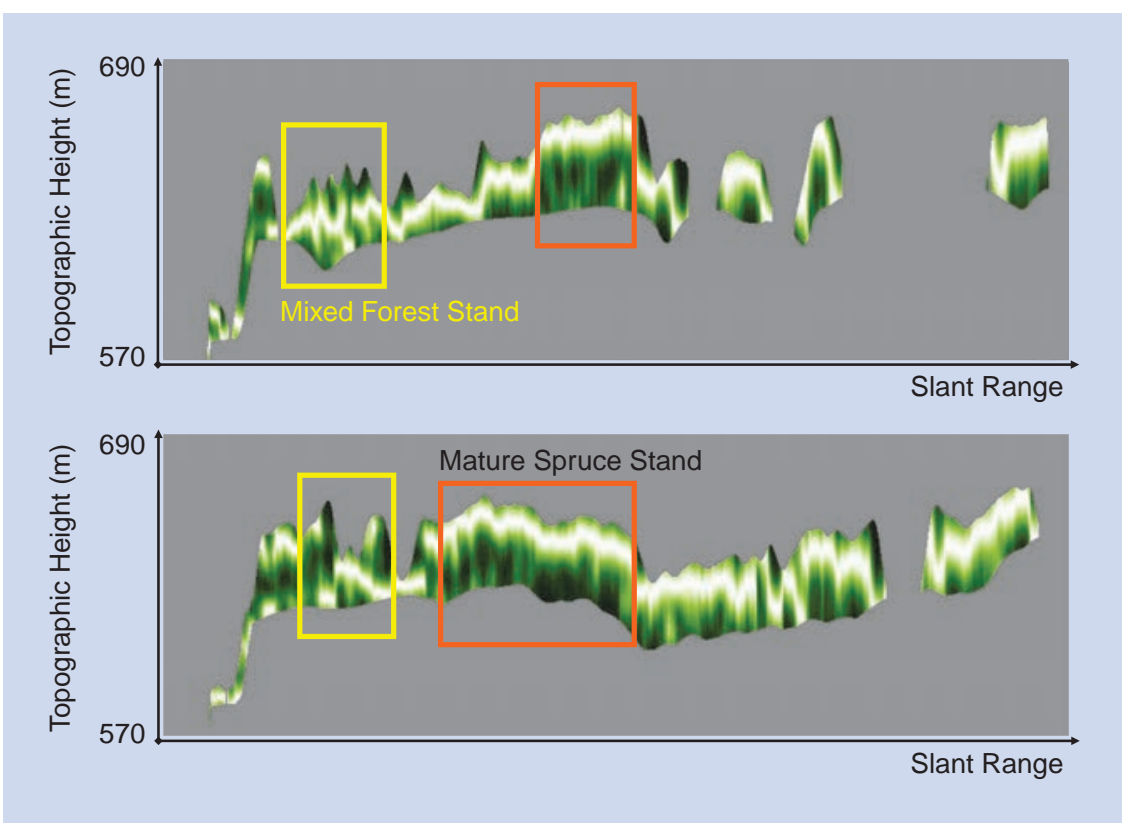

FIGURE 19. Vertical scattering profiles obtained from the inversion of dual-baseline Pol-InSAR data at L-band over the Traunstein test site. The profiles allow younger mixed (orange) and mature spruce stands (yellow) to be distinguished. 


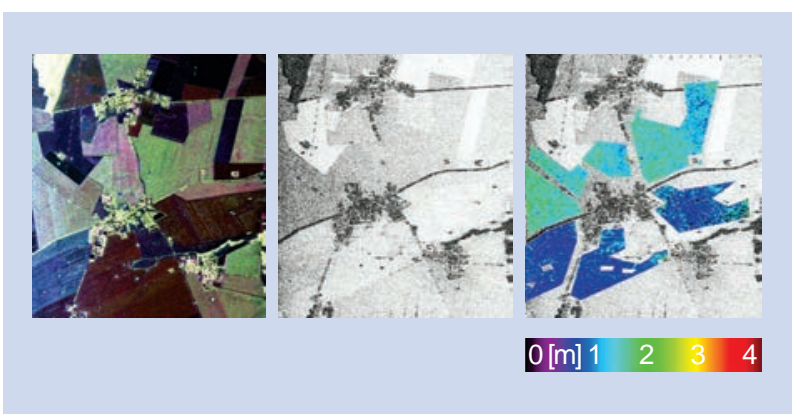

FIGURE 20. (a) Polarimetric Pauli RGB image with $R=|H H-V V|$ $G=2|H V|, B=|H H+V V|$. (b) Interferometric coherence image scaled from 0 (black) to one (white). (c) Crop height map obtained from the inversion of Pol-InSAR data acquired using large spatial baselines at L-band.

of stand conditions which is based on vertical structure components estimated from multibaseline Pol-InSAR data [51], [138], [139].

In contrast to forest vegetation applications, the development of quantitative agricultural vegetation Pol-InSAR applications is more challenging [116]-[118], [140]. The significant differences in vegetation height, form and attenuation values and in the propagation properties through the vegetation layer make the adoption of forest concepts for agriculture applications in many questions inappropriate. Opposed to forest applications, where lower frequencies are in general of advantage, in agricultural vegetation monitoring the use of high(er) frequencies that provide balanced volume and ground scattering contributions and therefore a better volume characterization is favored. However, lower frequencies are often preferred in repeat-pass acquisitions due to the higher temporal stability they provide. In this case large spatial baselines may compensate up to a certain degree the strong ground contribution and increase the sensitivity to the crop volume. Fig. 20 shows a crop height map obtained from the inversion of Pol-InSAR data acquired at L-band using large spatial baselines in the frame of the AGRISAR 2006 campaign [51]. The validation with ground measured crop heights shows an estimation performance on the order of $10-20 \%$.

The cryosphere is dominated by ice and snow scattering volumes. Admittedly, the understanding and development of Pol-InSAR applications with respect to ice and snow is today in a rather early stage [140]-[146]. The reason for this is the limited amount of experimental and validation data available as well as the limited understanding of the coherent interaction of electromagnetic waves with ice volumes. Of key interest is the characterization of the vertical distribution of scatterers within ice volumes as well as their seasonal and long-term dynamics especially in the percolation zone: There, the nearsurface vertical distribution of scatterers is influenced by seasonal melting processes leading to the presence of ice layers, lenses, and pipes, effecting the size and shape of ice crystals and trapped gas bubbles. In this direction a first remarkable result is the robust estimation of the scattering extinction profiles of ice volumes at different frequencies (L- and P-band) based on Pol-InSAR data acquired in the frame of the ICESAR 2007 campaign [144]. Fig. 21 shows the vertical structure profiles derived at X-, L- and P-band over the Ausfona Glacier in Svalbard, Norway.

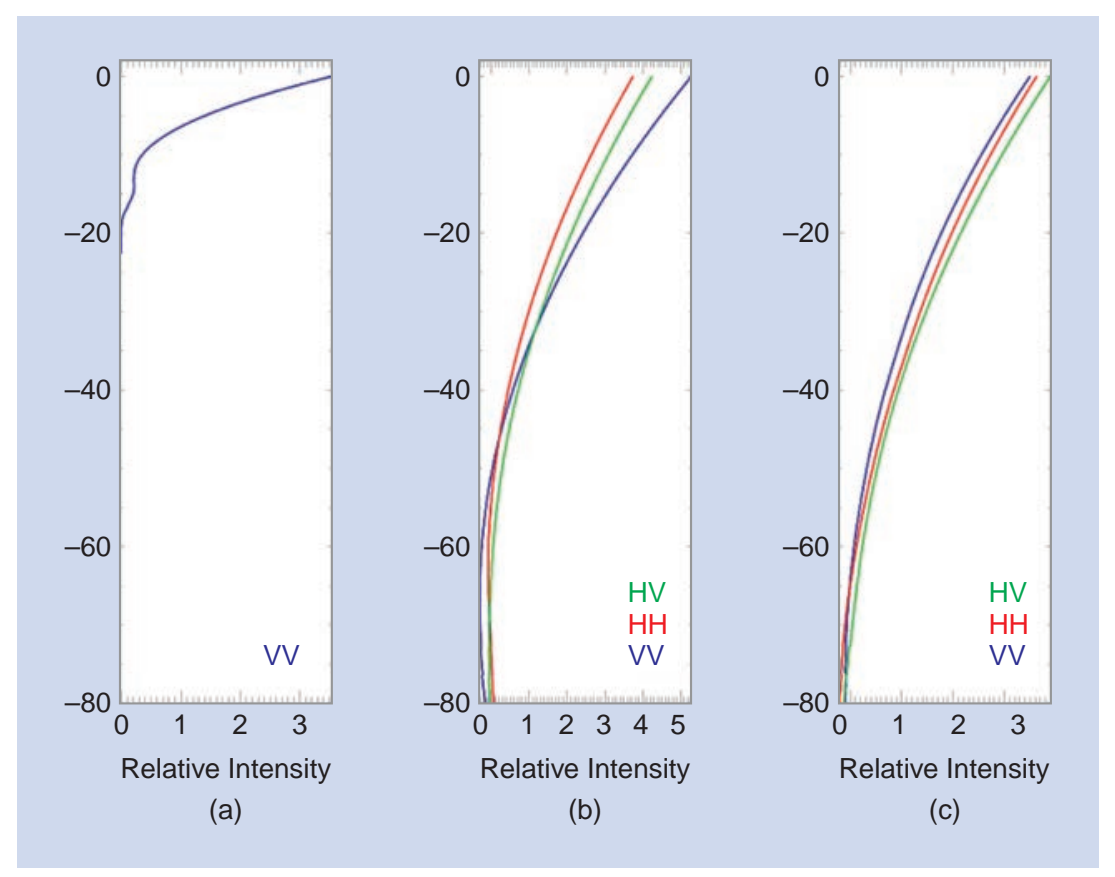

FIGURE 21. Vertical structure profiles derived at (a) X- , (b) L- and (c) P-band over the Ausfona Glacier in Svalbard, Norway.

\section{TOMOGRAPHY}

As commented in the previous section, the transmitted electromagnetic wave signal can penetrate through semi-transparent media, hence being sensitive to the volume of the observed objects. Forest is such a prominent volume scatterer, where at lower frequencies the transmitted signal penetrates-depending on the structure and dielectric properties of the forest-down to the ground even in very dense forests. However, due to the cylindrical geometry of a SAR sensor, targets at the same range distance cannot be separated and appear at the same resolution cell, i.e., they appear in layover. By adding a second acquisition, as InSAR does, it is possible to retrieve only a mean height of all backscattering contributions within the resolution cell. A significant improvement is given by 
Pol-InSAR, which however relies on a model in order to retrieve the vertical scattering distribution (see Section $\mathrm{V})$. Finally, in order to retrieve the whole vertical distribution of the scatterers in imaging terms, it is necessary to further exploit the synthetic aperture concept, which requires the use of multiple passes of the SAR sensor over the same area but at different positions, as depicted in Fig. 22. Such a concept is known as SAR tomography [19], [147]. One interesting application of SAR tomography is the imaging of vegetated areas. The resulting tomograms can be used, among other things, for the estimation of the ground-topography beneath the foliage, for the estimation of structural parameters, as well as for the detection of objects hidden beneath the foliage [19], [148], [149].

Similar as conventional SAR, the resolution in elevation is given by the length of the synthetic aperture in elevation, $L_{e l}$,

$$
\delta_{n}=\frac{\lambda r_{0}}{2 L_{e l}}
$$

where $n$ represents the elevation axis, and a repeat-pass scenario is implicitly assumed. Concerning the sampling requirements of the tomographic aperture, i.e., the distance between passes, one needs to consider the maximum spectral contributions of the received signal in order to accomplish Nyquist and avoid high sidelobes and ambiguities. Such frequency excursion in elevation depends on the total height of the volume in the direction perpendicular to LOS, $h_{V}$, as follows [19]

$$
\mu \leq \frac{\lambda r_{0}}{2 h_{V}}
$$

where $\mu$ is the minimum required distance between passes. In the frame of SAR tomography better resolution implies more passes, hence increasing the complexity of the whole acquisition geometry if resolutions in the same order of magnitude as the range and azimuth resolutions are to be obtained without undesired artifacts. As an example, the first experimental demonstration of SAR tomography [19] used 13 passes with an equidistant separation of $20 \mathrm{~m}$, reaching a resolution in elevation of $2.9 \mathrm{~m}$ at mid-range.

Figure 23 shows a tomogram over a forested area obtained with data acquired in 2006 by DLR's E-SAR airborne system in Dornstetten, Germany. The campaign was performed at L-band in quad-pol mode and a total of 21 tracks with an average baseline of $20 \mathrm{~m}$ were recorded. The tomogram clearly shows the different contributions and their polarimetric signatures, where the color coding corresponds to the elements of the Pauli vector (see (11)), namely, blue for the surface scattering $\left(S_{H H}+S_{V V}\right)$, red for the dihedral ground-trunk interaction $\left(S_{H H}-S_{V V}\right)$, and green for the volume of the canopy $\left(2 S_{X x}\right)$.

In opposition to airborne SAR sensors, spaceborne sensors can only reach a certain maximum separation between repeated acquisitions, especially with current

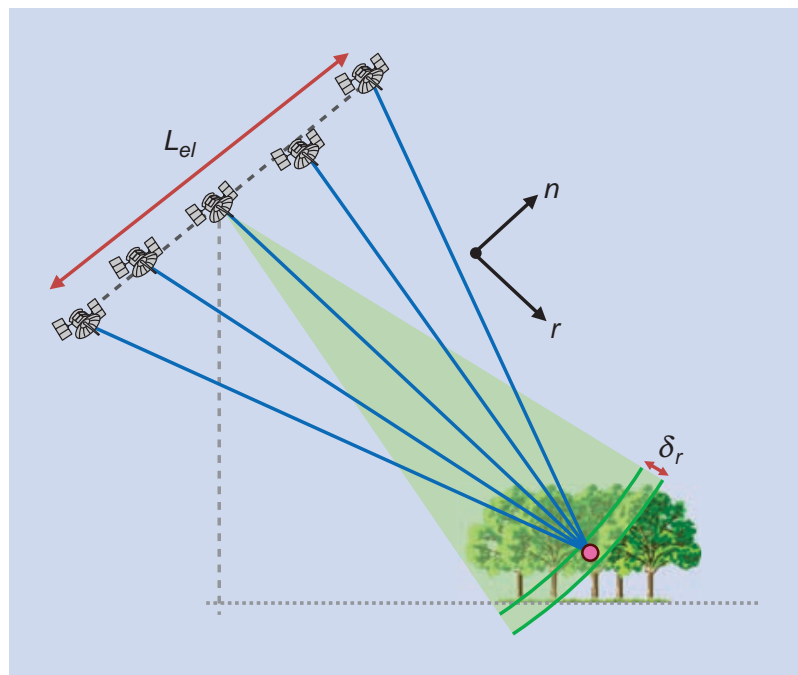

FIGURE 22. Acquisition geometry for tomographic imaging with a SAR sensor. $L_{e l}$ is the synthetic aperture in elevation and $n$ represents the elevation axis.

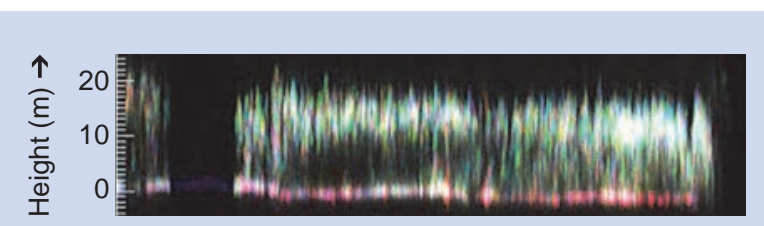

(a)

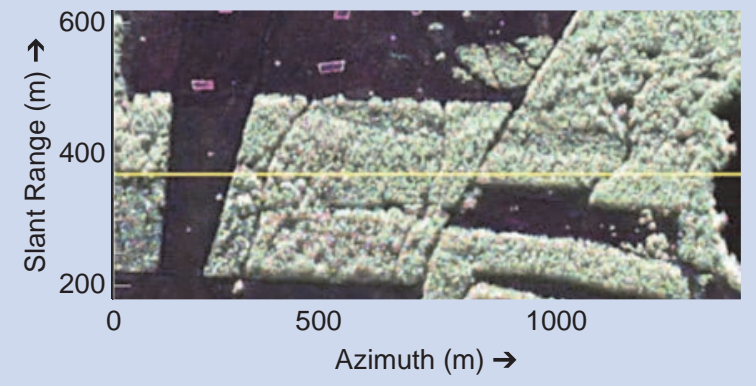

(b)

FIGURE 23. (a) Polarimetric tomographic profile over a forested area using DLR's E-SAR system at L-band [21]. The profile corresponds to the yellow line shown on the SAR in (b). A total of 21 tracks were acquired with an average baseline of $20 \mathrm{~m}$. Conventional beamforming, i.e., matched filtering, was used to focus the data. The color coding corresponds to the elements of the Pauli vector (see (11)), namely, blue for the surface scattering $\left(S_{H H}+S_{V V}\right)$, red for the dihedral ground-trunk interaction $\left(S_{H H}-S_{V V}\right)$, and green for the volume of the canopy $\left(2 S_{X X}\right)$.

single-satellite missions, since they are steered to follow small orbital tubes (250 $\mathrm{m}$ in the case of TerraSAR-X). Furthermore, the distribution of the acquisition geometry is in general non-uniform, hence resulting in an increase of the sidelobe level if conventional beamforming approaches are used to process the tomogram. The limited number of available samples together with the non-uniform 


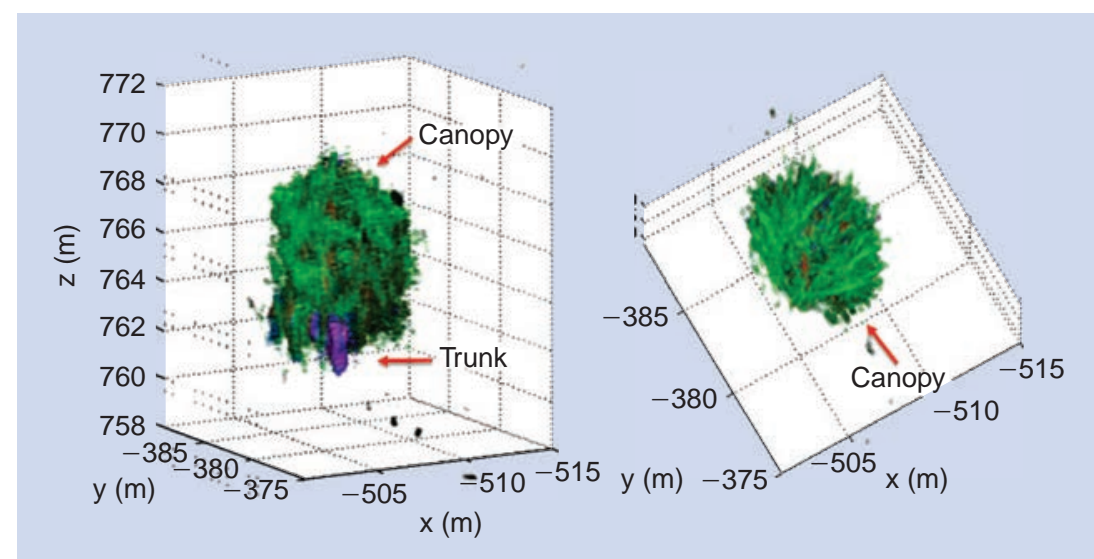

FIGURE 24. Fully polarimetric-holographic tomogram of a single tree in Pauli basis and focused with beamforming (BF). A total of 21 circular passes were processed into a volume and added coherently [166].

sampling call for more advanced processing schemes in order to increase the resolution beyond (47) and reduce the sidelobe level. The most commonly used solutions are based on MUSIC, CAPON, or, more-recently, compressive sensing (CS). MUSIC and CAPON are non-linear methods, while MUSIC is also parametric, i.e., it requires a priori information concerning the number of scatterers to be detected. Also, both SAR TOMOGRAPHY

EXPLOITS A SYNTHETIC APERTURE IN ELEVATION

TO RETRIEVE THE

VERTICAL DISTRIBUTION

OF SCATTERERS. require the estimation of the covariance matrix, implying a resolution loss due to the averaging operation. On the other hand, CS works at full resolution and can reconstruct non-uniformly sampled sparse signals, the latter meaning that the elevation profile to be estimated must be discrete. If the signal of interest is indeed sparse, the CS theory guarantees the possibility to obtain it at a rate significantly below the Nyquist one [150].

The use of CS in the frame of urban monitoring in combination with PS has been a topic of research in the recent years. Indeed, differential SAR tomography [20], [151]-[153] allows the discrimination of multiple scatterers in layover, e.g., ground and building facades, and at the same time the retrieval of their respective deformation velocities. These approaches have been mainly exploited using current high-resolution spaceborne sensors [106], [154]-[157], becoming a powerful tool for urban monitoring, as already mentioned in Section IV.

However, in the frame of forest monitoring, CS as such does not apply so well, as the elevation profile is indeed not sparse in the Fourier basis. One possible solution is to make use of a wavelet basis in order to obtain a sparse representation of the vertical structures, hence allowing the use of CS also for the imaging of forested areas [158].
A further possibility for tomographic imaging consists in acquiring data by means of circular trajectories instead of straight lines, the so-called circular SAR (CSAR) [159]-[165]. In CSAR, the sensor follows a $360^{\circ}$ circular flight around a spotted region, having 3-D imaging capabilities and allowing the maximum attainable resolution of a fraction of the wavelength in the ground plane. Nevertheless, both potentials are linked to the backscattering stability of the targets for different illumination angles, hence limiting the performance in real-world scenarios, where the targets tend to be anisotropic. In this sense, CSAR can also allow the study of the anisotropic properties of targets.

The acquisition of several circular trajectories over the same area further extends the potentials of CSAR, where several options are possible [162], [165], [166]. On the one hand, the circle can either be divided in small angular regions and a conventional tomographic processing be performed, so that the final combination of all sub-angle tomograms results in a holographic representation of the scene. A second option is to project each circle completely into a volume to finally add all of them coherently and achieve the maximum possible resolution. Fig. 24 shows an example of the latter approach with data of a multi-circular campaign performed with DLR's F-SAR sensor at L-band, where a total of 21 circular passes were acquired.

\section{FUTURE DEVELOPIMENTS}

The last decades have witnessed a tremendous increase of Earth observation applications that take advantage of the unique properties of high-resolution SAR images. To study dynamic processes on the Earth surface, more and more users ask for time series or stacks of coherent radar images acquired in repetition intervals that are as short as possible (Fig. 25, see also [167], [83]). The current generation of SAR instruments is, however, limited in their capability to acquire radar images with both high-resolution and wide-swath coverage. This immediately impacts the acquisition frequency if large contiguous areas shall be mapped systematically with a single satellite. The resolution versus swath width restriction is fundamental and closely connected to the intricacies of the SAR data acquisition process: SAR imaging exploits the Doppler shift arising from the sensor movement relative to the ground to improve the azimuth resolution well beyond the diffraction limit of the radar antenna. To achieve a high azimuth resolution, a broad Doppler spectrum has to be acquired. A broad Doppler spectrum means, in turn, that the system has to be operated with 


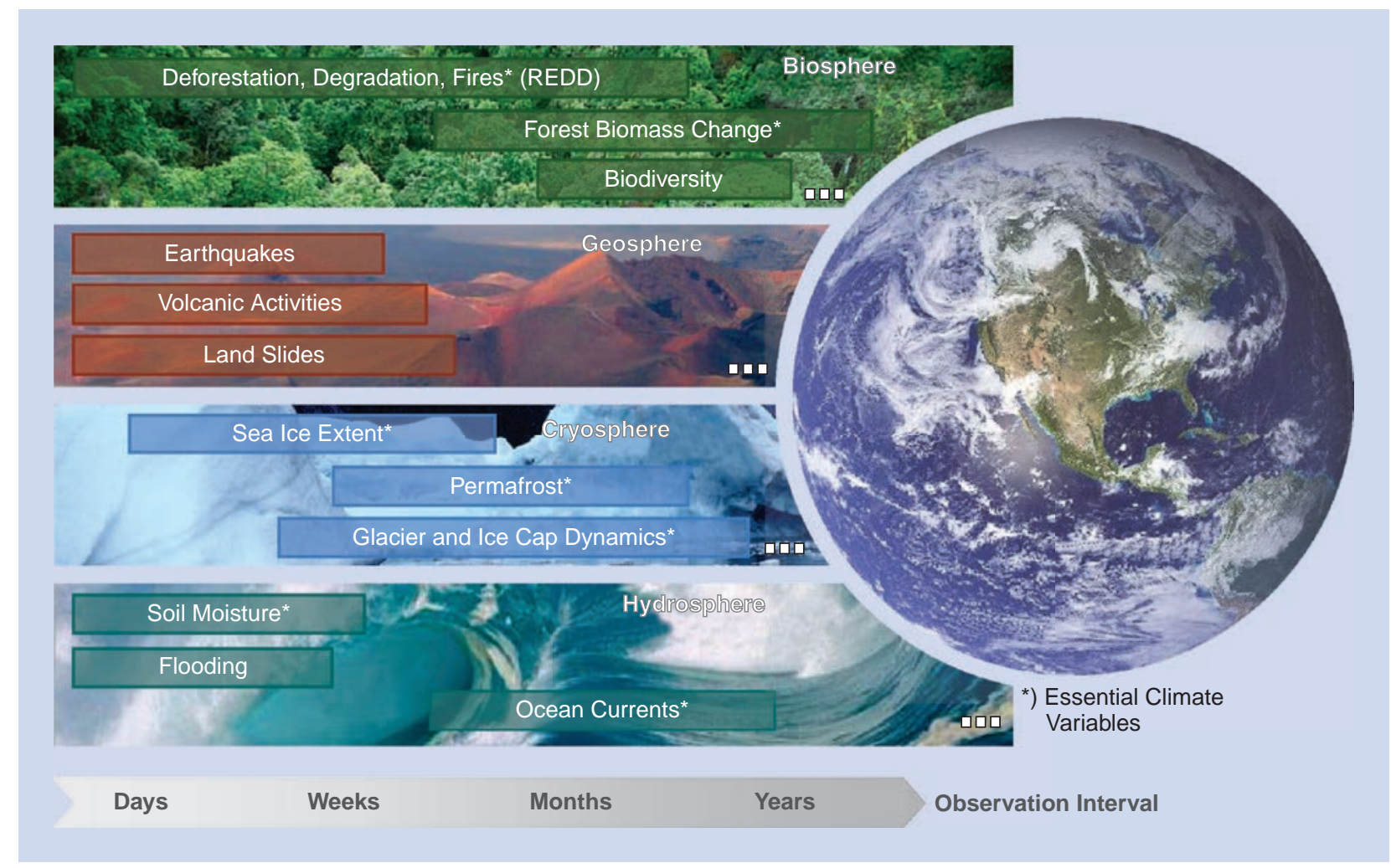

FIGURE 25. Requested observation intervals for the systematic monitoring of some exemplary dynamic processes on the Earth surface. Note that some processes may require multiple SAR data acquisitions for a single measurement to provide the required accuracy. An example is the estimation of forest biomass and its change by polarimetric SAR interferometry, where several interferometric acquisitions with varying cross-track baselines have to be combined to achieve high accuracy for different forest types and heights.

a high PRF to avoid azimuth ambiguities in the SAR imaging process. The high PRF limits, however, the unambiguous swath width since the range echo from the illuminated swath has to fit between two successive radar pulses to avoid range ambiguities. Wide unambiguous swath coverage and high azimuth resolution pose therefore contradicting requirements on the design of spaceborne SAR systems.

Several SAR imaging modes have been developed that provide different trade-offs between spatial coverage and azimuth resolution. Examples are the ScanSAR (or TOPS) mode, which enables a wide swath at the cost of an impaired azimuth resolution, and the Spotlight mode, which allows for an improved azimuth resolution at the cost of a noncontiguous imaging along the satellite track (see Section II). It is, however, up to now not possible to combine both imaging modes simultaneously in one and the same data take. To overcome this fundamental limitation, several innovative digital beamforming techniques have been suggested where the receiving antenna is split into multiple sub-apertures that are connected to individual receiver channels. The digitally recorded sub-aperture signals are combined in a spatiotemporal processor to simultaneously form multiple independent beams and to gather additional information about the direction of the scattered radar echoes. This information can be used to 1 ) suppress spatially ambiguous signal returns from the ground by null-steering, 2) increase the receiving gain without a reduction of the imaged area by switching between narrow, high gain beams, 3) suppress spatially localized interferences by space-time adaptive processing, and 4) gain additional information about the dynamic behavior of the scatterers and their surroundings.

\section{A. HIGH-RESOLUTION WIDE-SWATH IMAGING}

Digital beamforming and multi-aperture signal recording are key technologies that will boost the performance of future SAR systems by at least one order of magnitude. These improvements are evident, both from research activities and demonstration campaigns [168]-[196]. A prominent example is the high-resolution wide-swath (HRWS) SAR [172] which is currently under development 


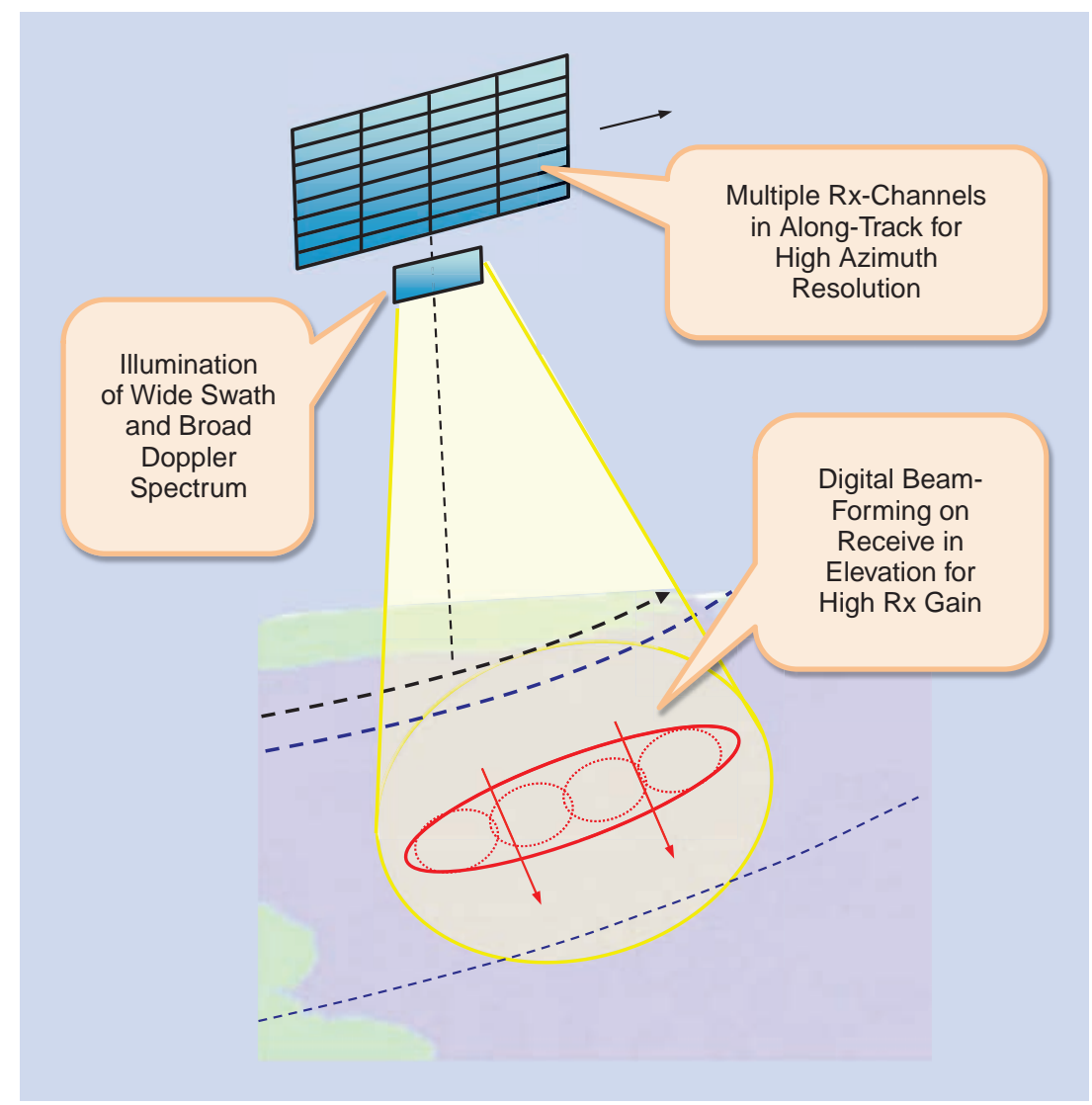

FIGURE 26. High-resolution wide-swath (HRWS) SAR system. The displaced phase center technique in azimuth is combined with real-time digital beamforming on receive in elevation to overcome fundamental limitations of conventional SAR systems. This enables the mapping of a wide image swath with high geometric resolution. In the meantime this concept has been extended to use the same antenna for transmit and receive.

angular range. Since there exists a one-to-one relationship between the radar pulse travel time and its angle of arrival, one can maximize for each instant of time the gain of a receiving antenna array by a real-time beamforming process that was first described in [168]. This technique is denoted scan-on-receive (SCORE) in the HRWS system, where it was further refined by a kind of dispersive $\mathrm{Rx}$ beam to fully collect the echoes also for the case where long chirped pulses are transmitted. A large receiving antenna can hence be used to improve the system sensitivity without narrowing the swath width. This enables in turn a reduced transmit power and allows for longer operation times during each orbit. Further modifications may be necessary to avoid notable gain losses if mountainous terrain shall be mapped with large Rx apertures [178], [183]. Topography variations have moreover to be considered for accurate radiometric calibration.

The unambiguous swath width of the HRWS system is, similar to a conventional stripmap SAR, limited by the antenna length. To improve the azimuth resolution beyond that of a classical stripmap SAR (where the resolution is typically some-

at EADS Astrium with support from the German Aerospace Center (DLR). This system has been specified to map a $70 \mathrm{~km}$ wide swath with a resolution of $1 \mathrm{~m}$, thereby exceeding the number of acquired ground resolution cells of the TerraSAR-X stripmap mode ( $3 \mathrm{~m}$ resolution at $30 \mathrm{~km}$ swath width) by a factor of 21 . To achieve this improvement, the HRWS system employs two advanced techniques: Digital beamforming on receive in elevation and multiple aperture recording in azimuth (cf. Fig. 26).

Digital beamforming on receive is used to steer in real-time a narrow elevation

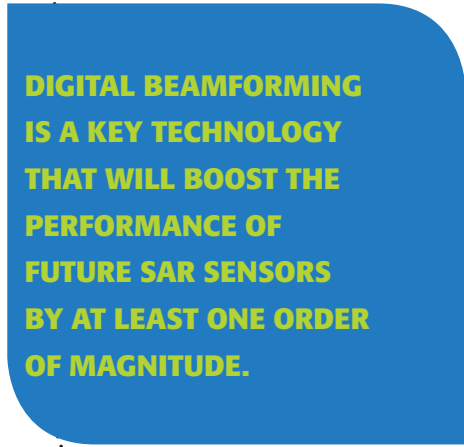
beam towards the direction from which the radar echo from the ground is expected to arrive. By this, the system exploits the imaging geometry of a side-looking radar where the scattered echoes from the ground arrive at each instant of time as a superposition of plane waves from a rather narrow what larger than half the antenna length) the receiving antenna is divided into multiple subapertures that are mutually displaced in the along-track direction. Each azimuth subaperture is connected to an individual receiver channel and the recorded signals are transmitted to the ground for further processing [169]. By this, multiple azimuth samples can be acquired for each transmitted pulse, while each subaperture sees a wider Doppler spectrum. For this, a short transmit antenna or an appropriate beamspoiling are used to illuminate a wide footprint on the ground. The coherent combination of the subaperture signals enables then the unambiguous generation of a high-resolution SAR image beyond the classical limitations. The original conception of this technique required a fixed PRF such that the satellite moved just one half of the overall antenna length between successive transmit pulses [169]. This restriction could be overcome with the development of a dedicated multichannel SAR processing that was first introduced in [176] and then further analyzed and elaborated in [182]. The suitability of this technique has in the meantime been successfully demonstrated in airborne campaigns [191] and 
recently also in a spaceborne experiment using the dual receive antenna mode of TerraSAR-X [196].

\section{B. ADVANCED CONCEPTS FOR ULTRA-WIDE SWATH IMAGING}

The HRWS system from the previous section requires a very long antenna to map an ultra-wide swath with high azimuth resolution. As a rule of thumb, a length of $10 \mathrm{~m}$ is required for every $100 \mathrm{~km}$ swath extension. To avoid an undue increase of the antenna length, several new instrument architectures and modes have been suggested in [179]. One example is the combination of the displaced phase center technique described in the previous section with a ScanSAR or TOPS mode (cf. Fig. 27, top left). As in classical ScanSAR, azimuth bursts are used to map several swaths. The associated resolution loss from sharing the synthetic aperture among different swaths is compensated by illuminating a wider Doppler spectrum and collecting the radar echoes with multiple displaced azimuth apertures. Such a system is currently considered by ESA as a possible successor to Sentinel-1 [188]. The goal is to map a $400 \mathrm{~km}$ wide swath with $5 \mathrm{~m}$ resolution. The peculiarities of the multichannel ScanSAR processing and their impact on system performance have been analyzed in [186]. A possible drawback of this multichannel ScanSAR approach is the rather high Doppler centroid with which some targets will be mapped if a high resolution is desired. The situation becomes even worse in a multichannel TOPS mode. High squint angles may moreover challenge co-registration for SAR interferometry [197].

Besides multichannel ScanSAR, several alternative concepts have been suggested in [179]. A common feature of these alternatives is that they record not only one but several radar echoes arriving simultaneously from different directions. For this, multiple narrow elevation beams are formed where each beam follows the echo of a different pulse transmitted by a wide beam illuminator. This enables an increase of the coverage area without the necessity to either lengthen the antenna or to employ burst modes. The top right of Fig. 27 provides an illustration, where three narrow $\mathrm{Rx}$ beams follow the echoes from three simultaneously mapped image swaths that are illuminated by a broad Tx beam. A sufficiently high antenna is needed to separate the echoes from the different swaths by digital beamforming on receive. An alternative is range variant null steering as already suggested for the quad-element array SAR in [171]. Since the azimuth resolution is, as in a classical stripmap SAR, given by half of the antenna length, this will typically lead to a shorter but higher SAR antenna. Such a more compact shape may have constructive advantages, avoiding, e.g., a complicated folding for the satellite launch. Note that this mode makes also effective use of the hardware already available for digital beamforming in elevation and avoids its duplication to implement multiple azimuth channels. The required ultra-wide swath illumination can either be accomplished by a separate small Tx antenna, or by using a combined Tx/ Rx antenna together with (a) phase tapering, (b) spectral Tx diversity or (c) an illumination with a sequence of sub-pulses [178].

An interesting alternative to a planar antenna is a reflector that is fed by a multichannel array as illustrated on the lower left of

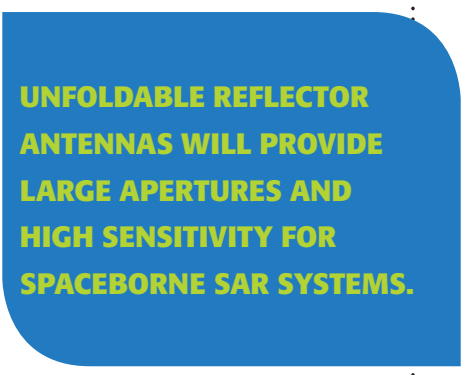
Fig. 27. A parabolic reflec-

tor focuses an arriving plane wave on one or a small subset of feed elements. As the swath echoes arrive as plane waves from increasing look angles, one needs only to read out one feed element after the other to steer a high gain beam in concert with the arriving echoes. This technique was originally suggested in [170] and then reinvented independently by DLR and NASA/JPL during a

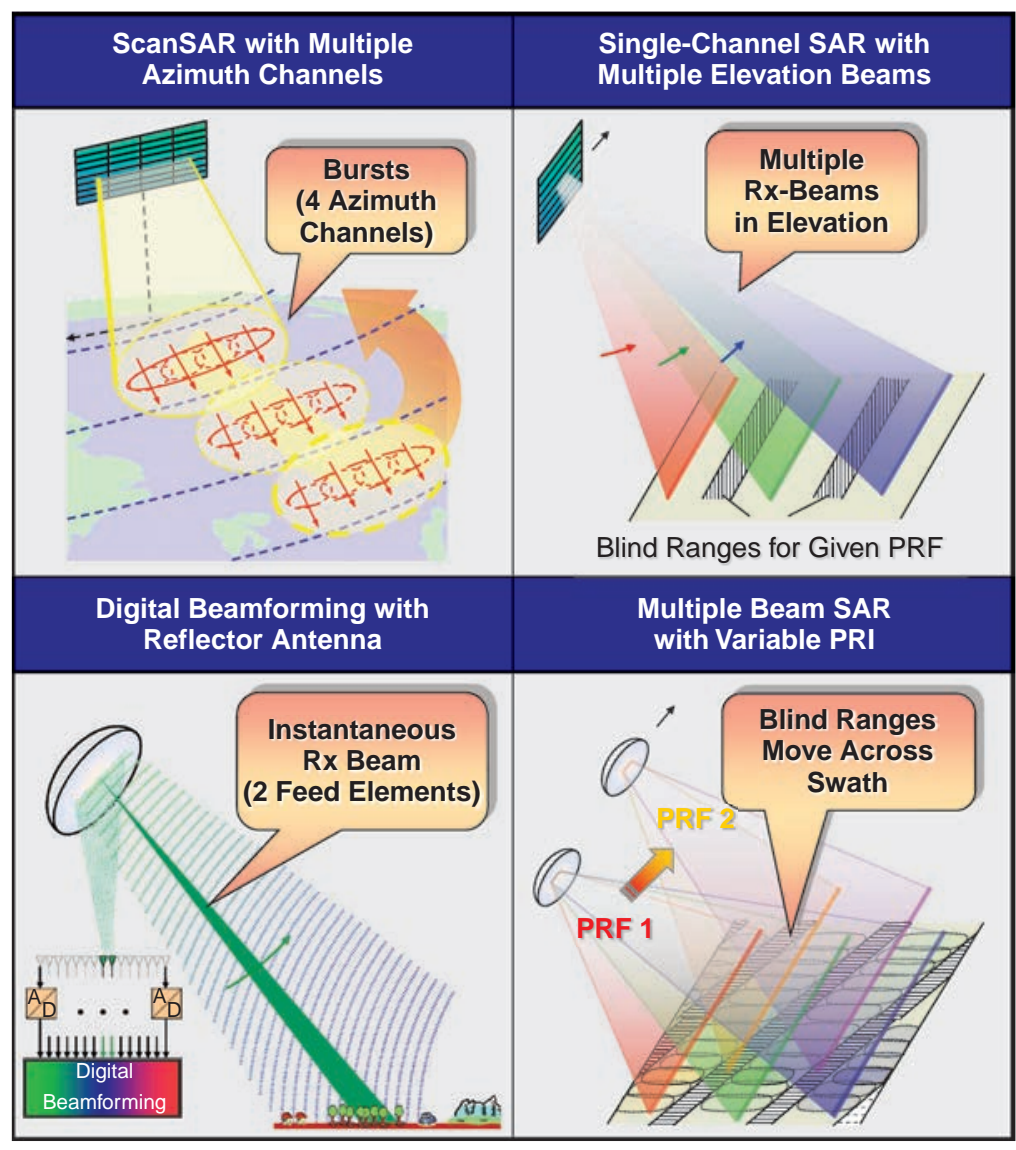

FIGURE 27. Advanced instrument architectures for ultra-wide swath SAR imaging. 


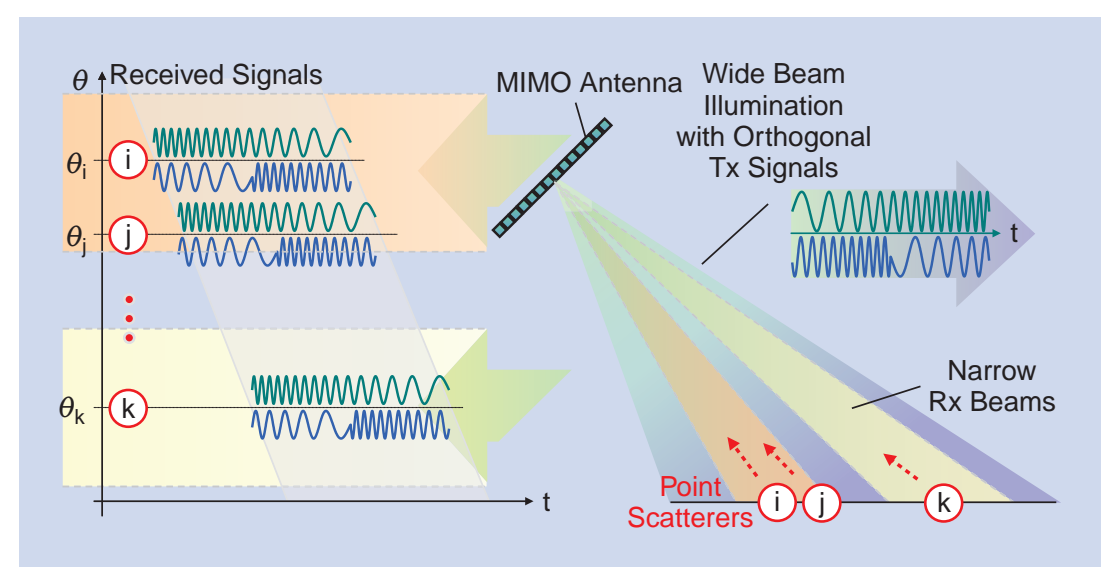

FIGURE 28. MIMO-SAR based on two short-term shift-orthogonal transmit signals. The Tx waveforms are designed such that their cross-correlation vanishes for small time offsets (as it applies to scatterers i and j). For larger time offsets, the otherwise correlated signal returns are separated via narrow Rx beams (as it applies to scatterers i and $\mathrm{k}$ ). This exploitation of the side-looking imaging geometry together with appropriately designed transmit waveforms enables the simultaneous use of multiple Tx signals and the unambiguous separation of their radar echoes also in the realistic case of a distributed scatterer scenario. Note that both waveforms have the same spectral support.

joint Tandem-L/DESDynI study [83]. JPL suggested an analogue switching between the feed elements [185], while DLR argued in favor of connecting each feed element with its own A/D converter [179]. The former may allow a cheaper implementation, while the latter enables a performance improvement and increased flexibility by combining multiple feed signals in a digital signal processor [184], [192]. The solution with the digital feed is also in favor of more advanced modes like the multiple-beam technique introduced above. Compared to a direct radiating array, the computational requirements for real-time beamsteering are significantly reduced since only few feed signals have to be combined at each instant of time.

A drawback of the multi-beam mode are the blind ranges that are due to the fact that the radar cannot transmit and receive at the same time. This can be overcome in a bistatic SAR where the transmitter is sufficiently separated from the receiver [175]. To avoid a separate transmit satellite, one can employ a variation of the PRF which shifts the blind ranges across the swath (Fig. 27, lower right). The PRF variation could either be implemented in discrete steps leading to a multiple-beam ScanSAR mode or a pulse-topulse variation of the PRI. The latter provides better performance but requires a dedicated SAR processing which is currently under development [193].

\section{MULTIPLE-INPUT MULTIPLE-OUTPUT (MIMO) SAR}

A receiver with $n$ subapertures allows a simultaneous sampling of the arriving wavefronts with $n$ phase centers. The effective number of phase centers can be increased by using additional transmitters [180]. Such an extension was already suggested for resolution improvement in the context of a forward looking imaging radar experiment on a helicopter [173] and later elaborated in more detail for a 3-D imaging system on a UAV [177]. In a SAR system, the possible benefits of using multiple transmitters range from an increase of the coverage area to the suppression of range and azimuth ambiguities to the provision of additional baselines for interferometric or even tomographic applications [178], [181], [189].

To separate the echoes from the different transmitters, some publications suggest to simultaneously transmit mutually orthogonal waveforms [190], [194]. While this condition allows perfect separation of the scattered waveforms from a single point target, it is not sufficient to separate the signals in case of an extended scattering scenario [178], [198]. The reason is that the orthogonality is not ensured for arbitrary shifts between the transmit signals. As a result, the energy from spatially separated scatterers illuminated by the other waveform(s) will not vanish after range focusing but appear either smeared or at different positions. This reasoning is evident by considering range focusing as an all-pass filter in the frequency domain. Depending on the number of transmitters, the smeared energy from the orthogonally illuminated scatterer ensemble may even exceed the focused target impulse response [195].

As an alternative, it was suggested to employ transmit waveforms that are mutually orthogonal for arbitrary shifts. This implies, however, that the waveforms cover disjunct frequency bands which prevents their use for many of the aforementioned applications which rely on a coherent combination of spectrally overlapping signals. To avoid this deterioration and to allow nevertheless a coherent combination of multiple transmit signals within the same frequency band, it has been suggested in [178], [198], [199] to employ especially designed waveforms (e.g., chirp-based OFDM signals) in combination with digital beamforming on receive. The basic idea behind this approach can be expressed by a short-term shiftorthogonality condition where two different transmit signals are required to be only orthogonal for a subset of mutual shifts which are typically provided by a symmetric interval $\left[-\tau_{s}, \tau_{s}\right]$. The short-term shift-orthogonality enables a perfect separation of the echoes from neighboring scatterers as long as the relative radar signal delay is within the interval $\left[-\tau_{s}, \tau_{s}\right]$. The echoes from more distant scatterers can then be separated by digital beamforming on receive as illustrated in Fig. 28. For this, the antenna should be of sufficient height and it may again be advantageous to use a large reflector instead of a planar array antenna to provide the required aperture. 


\section{SUMMARY AND OUTLOOK}

\section{SUMMARY}

This paper provides a tutorial on Synthetic Aperture Radar systems with a focus on the basic theory, imaging techniques, applications and future technologies. Looking back 30 years ago, the remote sensing community

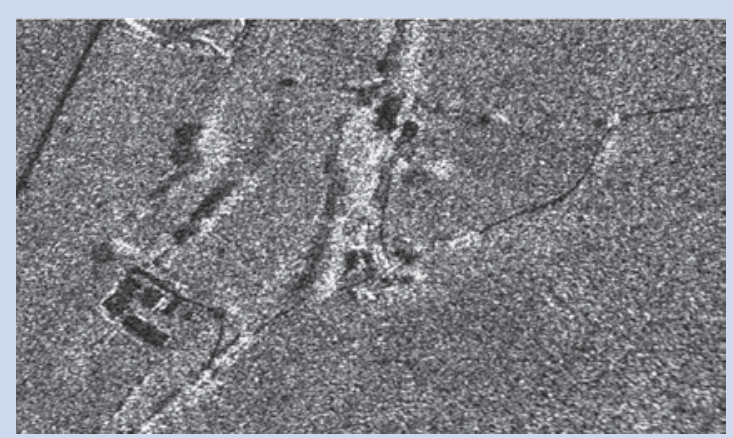

(a)

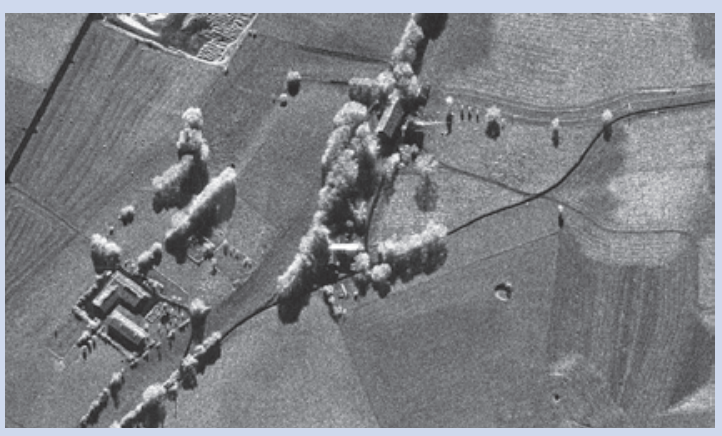

(b)

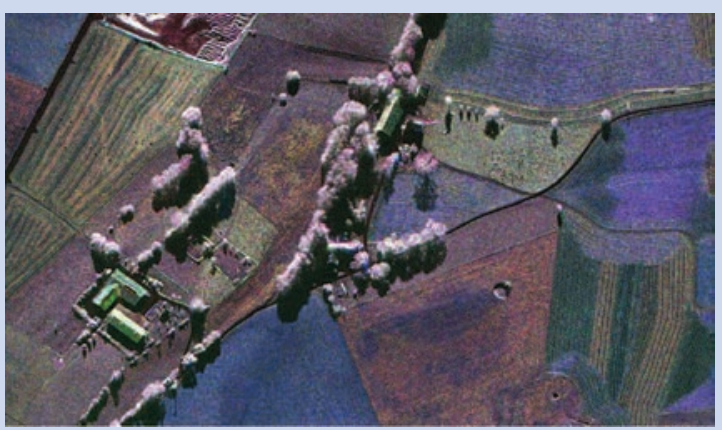

(c)

FIGURE 29. Comparison of the increase in the information content achieved by improved geometric resolution (system bandwidth) and multi-channel operation (polarimetry). (a) X-band image with $1 \mathrm{~m}$ resolution (state of the art of spaceborne $X$-band sensors in Spotlight imaging mode); (b) and (c) X-band image with $0.25 \mathrm{~cm}$ resolution without and with a fully polarimetric imaging mode, respectively (simulation of the data quality to be provided by the next generation of spaceborne X-band sensors). Imaged area: Kaufbeuren, Germany. The images in (b) and (c) were acquired by the F-SAR sensor of DLR [21]. was postulating statements about "the promise of radar remote sensing." While at that time the Landsat series of satellites was already operational and widely used for many applications, SAR remote sensing was just in its infancy. There was a big expectation about the future of radar remote sensing. In the 90 s a paradigm shift occurred in the SAR development from technology push to the user demand pull. We have now entered into a golden age for spaceborne SAR with more than 10 satellites to be launched within the next 5 years. Many applications became mature and are an integral part in operational services of geo-information products as well as in geoscience and climate research. Spaceborne SAR sensors have the only technology that can provide all-weather day-and-night high-resolution information about Earth's dynamic processes on a global scale. Table 7 summarizes a compilation of application examples which are classified in the following categories: Land, vegetation, ocean, sea ice, snow and land ice.

As described in Sections III to V, the research on information retrieval from SAR images clearly shows a trend for using model-based inversion algorithms. The observation interval requirements for the SAR sensor vary from hours to a day (e.g., disaster monitoring), over a day to weeks (e.g., soil moisture, agriculture) up to years and decades (e.g., subsidence, eco-systems, climate change). In a general sense, the reliability and robustness of the information retrieval increase with the amount of observables and information content available in the data. This increase in the information content can be achieved by several

TABLE 7. COMPILATION OF INFORMATION EXTRACTION AND APPLICATION EXAMPLES FOR SAR IMAGERY. ESSENTIAL CLIMATE VARIABLES (ECV) AS DEFINED BY THE INTERGOVERNMENTAL PANEL ON CLIMATE CHANGE (IPCC) ARE IDENTIFIED WITH "*".

Land Multi-purpose land surface imagery, soil type, land cover*, Earth topography (elevation and surface model), lake levels*, subsidence, landslides, erosion, earthquake and volcano monitoring, disaster monitoring, mitigation and assessment, flooding monitoring, coherent change detection, urban and infrastructure planning, road traffic monitoring, soil moisture*, wetlands monitoring, permafrost and seasonally-frozen ground*

Vegetation Vegetation type, forest biomass*, forest biomass change, biodiversity, forest profile, forest height, fire disturbance and monitoring, crop classification, crop height, crop biomass, deforestation and forest degradation

Ocean Multi-purpose ocean imagery, sea state*, ocean currents*, wind speed and vector over sea surface, bathymetry at coastal zones, wave height, ocean wavelength, wave direction, oil spill cover, ship monitoring

Sea ice Sea-ice cover and extent, sea-ice type, sea-ice thickness, iceberg cover and movement, ship route optimization

Snow and Snow cover*, ice and glacier cover*, snow melting status land ice (wet/dry), snow water equivalent, glacier motion and dynamics, glacier topography 
(a)

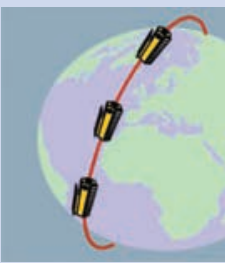

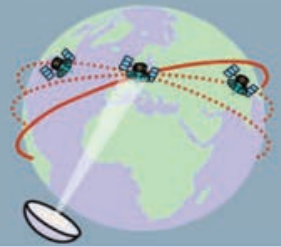

(b)

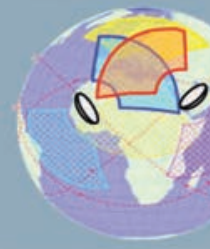

(c)
FIGURE 30. Concepts for a future constellation of radar satellites for quasi-continuous monitoring of the Earth. (a) Low Earth Orbit (LEO) satellites, (b) Geostationary Earth Orbit (GEO) transmitter with a large reflector and LEO receivers, and (c) Medium Earth Orbit (MEO) satellites using a large reflector.

means including the use of time series, space diversity (interferometry, tomography), multi-channel acquisition (polarimetry, multi-frequency) and signal bandwidth (resolution) as well as a combination of those. Each application poses however a different requirement on the sensor characteristics and observation scenario. The forward modeling followed by an inversion and regularization is a key procedure to help defining the sensor characteristics and observation scenario for each application. Fig. 29 shows a clear example of the increase in the information content provided by SAR images with 1 meter resolution (state of the art) compared to $25 \mathrm{~cm}$ resolution (next generation of spaceborne X-band SAR systems to be launched within the next years) using a single-channel and a polarimetric SAR sensor. Last but not least, data fusion with different sensor types can be further explored towards the use of complementary information in the multi-dimensional data space.

Looking now at the state of the art of SAR systems and applications, there is no doubt that the promise of SAR remote sensing has been fulfilled. Today, spaceborne SAR is an integral part of a multitude of sensors for Earth remote sensing which provide not only the required information to monitor and predict the changes in the environment and Earth system processes, but also to deliver an archive of the history of the Earth. Further, spaceborne remote sensing delivers an essential contribution to solve several societal challenges such as climate change, sustainable development, mobility, environmental protection and security.

\section{VISION FOR SAR REMOTE SENSING}

In a changing and dynamic world, high-resolution and timely geospatial information with global access and coverage becomes increasingly important. Constellations of radar satellites will play a major role in this task, since spaceborne radar is the only sensor that has allweather, day-and-night, high-resolution imaging capability. Examples of applications for such a constellation are environmental remote sensing, road traffic, hazard and disaster monitoring, geoscience and climate research, 3-D and 4-D mapping as well as reconnaissance and security related tasks.

The vision for spaceborne SAR remote sensing looks exciting. A space-based sensor web will provide a view of our planet like we are used to see with Google Earth, but with high-resolution images and relevant geospatial information being updated every few minutes. Future SAR mission concepts with digital beamforming technology in combination with large reflector antennas are being developed which will outperform the imaging capacity of current SAR systems by at least one order of magnitude [168]-[196]. These highly innovative concepts will allow the global observation of dynamic processes on the Earth's surface with hitherto unknown quality and resolution. New spaceborne radar technologies will also allow the implementation of a constellation of radar satellites for reliable and systematic monitoring of the Earth's surface (cf. Fig. 30). It will open the door to a future global remote sensing system for the continuous observation of dynamic processes over the Earth, as it currently exists for weather prediction, where a network of geostationary satellites is used.

\section{REFERENCES}

[1] F. Henderson and A. Lewis, Manual of Remote Sensing: Principles and Applications of Imaging Radar. New York: Wiley, 1998.

[2] J. C. Curlander and R. N. McDonough, Synthetic Aperture Radar: Systems and Signal Processing. New York: Wiley, 1991.

[3] C. Elachi and J. van Zyl, Introduction to the Physics and Techniques of Remote Sensing. New York: Wiley, 2006

[4] K. Tomiyasu, "Tutorial review of synthetic-aperture radar (SAR) with applications to imaging of the ocean surface," Proc. IEEE, vol. 66, no. 5, May 1978

[5] C. Oliver and S. Quegan, Understanding Synthetic Aperture Radar Images. Herndon, VA: SciTech Publishing, 2004.

[6] R. Raney, "Theory and measure of certain image norms in SAR," IEEE Trans. Geosci. Remote Sensing, vol. 23, no. 3, May 1985.

[7] I. Woodhouse, Introduction to Microwave Remote Sensing. Boca Raton, FL: CRC Press, 2006

[8] G. Franceschetti and R. Lanari, Synthetic Aperture Radar Processing. Boca Raton, FL: CRC Press, 1999.

[9] I. G. Cumming and F. H. Wong, Digital Processing of Synthetic Aperture Radar Data: Algorithms and Implementation. Norwood, MA: Artech House, 2005.

[10] C. A. Wiley, "Pulsed doppler radar methods and apparatus," U.S. Patent 3196 436, 1954

[11] C. Wiley, "Synthetic aperture radars: A paradigm for technology evolution," IEEE Trans. Aerosp. Electron. Syst., vol. 21, no. 3, pp. 440-443, May 1985 
[12] L. Cutrona, W. Vivian, E. Leith, and G. Hall, "Synthetic aperture radars: A paradigm for technology evolution," IRE Trans. Military Electron., pp. 127-131, Apr. 1961.

[13] P. A. Rosen, S. Hensley, I. R. Joughin, F. K. Li, S. N. Madsen, E. Rodriguez, and R. M. Goldstein, "Synthetic aperture radar interferometry," Proc. IEEE, vol. 88, no. 3, pp. 333-382, Mar. 2000.

[14] D. Massonnet and J. Souryis, Imaging with Synthetic Aperture Radar. EPFL Press, 2008.

[15] J.-S. Lee and E. Pottier, Polarimetric Radar Imaging: From Basics to Applications. Boca Raton, FL: CRC Press, 2009.

[16] D. Massonnet, M. Rossi, C. Carmona, F. Adragna, G. Peltzer, K. Feigl, and T. Rabaute, "The displacement field of the Landers earthquake mapped by radar interferometry," Nature, vol. 364, pp. 138-142, 1993.

[17] A. Ferretti, C. Prati, and F. Rocca, "Permanent scatterers in SAR interferometry," IEEE Trans. Geosci. Remote Sensing, vol. 39, no. 1, pp. 8-30, Jan. 2001.

[18] K. P. Papathanassiou and S. R. Cloude, "Single-baseline polarimetric SAR interferometry," IEEE Trans. Geosci. Remote Sensing, vol. 39, no. 11, pp. 2352-2363, Nov. 2001.

[19] A. Reigber and A. Moreira, "First demonstration of airborne SAR tomography using multibaseline L-band data," IEEE Trans. Geosci. Remote Sensing, vol. 38, no. 5, pp. 2142-2152, Sept. 2000.

[20] G. Fornaro, A. Pauciullo, D. Reale, X. Zhu, and R. Bamler, "SAR tomography: an advanced tool for 4D spaceborne radar scanning with application to imaging and monitoring of cities and single buildings," IEEE Geosci. Remote Sensing Newslett., pp. 10-18, 2012.

[21] A. Reigber, R. Scheiber, M. Jäger, P. Prats-Iraola, I. Hajnsek, T. Jagdhuber, K. Papathanassiou, M. Nannini, E. Aguilera, S. Baumgartner, R. Horn, A. Nottensteiner, and A. Moreira, "Very-high-resolution airborne synthetic aperture radar imaging: Signal processing and applications," Proc. IEEE, vol. 101, no. 3, Mar. 2013.

[22] (2012, Oct.) Catalogue of satellite instruments. CEOS EO Handbook. [Online]. Available: http://www.eohandbook.com

[23] C. Elachi, Spaceborne Radar Remote Sensing: Applications and Techniques. New York: IEEE Press, 1988.

[24] A. Freeman, "SAR calibration: An overview," IEEE Trans. Geosci. Remote Sensing, vol. 30, no. 6, pp. 1107-1121, Nov. 1992.

[25] C. Cafforio, C. Prati, and F. Rocca, "SAR data focusing using seismic migration techniques," IEEE Trans. Aerosp. Electron. Syst., vol. 27, no. 2, pp. 194-207, Mar. 1991.

[26] A. Reigber, E. Alivizatos, A. Potsis, and A. Moreira, "Extended wavenumber domain SAR focusing with integrated motion compensation," IEE Proc. Radar, Sonar Navigat. (1994-2006), vol. 153, no. 3, pp. 301-310, June 2006.

[27] C. Wu, K. Y. Liu, and M. Jin, "Modeling and a correlation algorithm for spaceborne SAR signals," IEEE Trans. Aerosp. Electron. Syst., vol. 18, no. 5, pp. 563-574, Sept. 1982.

[28] M. Y. Jin and C. Wu, "A SAR correlation algorithm which accommodates large-range migration," IEEE Trans. Geosci. Remote Sensing, vol. 22, no. 6, pp. 592-597, Nov. 1984.

[29] C. Y. Chang, M. Y. Jin, and J. C. Curlander, "SAR processing based on the exact two-dimensional transfer function," in Proc. IEEE Int. Geoscience and Remote Sensing Symp. (IGARSS), Houston, TX, 1995.

[30] R. K. Raney, H. Runge, R. Bamler, I. Cumming, and F. H. Wong, "Precision SAR processing using chirp scaling," IEEE Trans. Geosci. Remote Sensing, vol. 32, no. 4, pp. 786-799, July 1994.

[31] A. Moreira, J. Mittermayer, and R. Scheiber, "Extended chirp scaling algorithm for air- and spaceborne SAR data processing in stripmap and ScanSAR imaging modes," IEEE Trans. Geosci. Remote Sensing, vol. 34, no. 5, pp. 1123-1136, Sept. 1996.

[32] W. G. Carrara, R. S. Goodman, and R. M. Majewski, Spotlight Synthetic Aperture Radar: Signal Processing Algorithms (IPF). Norwood, MA: Artech House, 1995.
[33] F. De Zan and A. Monti Guarnieri, "TOPSAR: Terrain observation by progressive scans," IEEE Trans. Geosci. Remote Sensing, vol. 44, no. 9, pp. 2352-2360, Sept. 2006.

[34] (2013). POLinSAR. 6th International Workshop on Science and Applications of SAR Polarimetry and Polarimetric Interferometry. Frascati, Italy, 2013. [Online]. Available: https://earth.esa.int/ web/guest/polinsar-2013

[35] S. R. Cloude, Polarisation: Applications in Remote Sensing. New York: Oxford Univ. Press, 2009.

[36] G. Sinclair, "The transmission and reception of elliptically polarized waves," Proc. IRE (through 1962), vol. 38, no. 2, pp. 148-151, 1950.

[37] P. Dubois-Fernandez, H. Cantalloube, B. Vaizan, G. Krieger, R. Horn, M. Wendler, and V. Giroux, "ONERA-DLR bistatic SAR campaign: Planning, data acquisition, and first analysis of bistatic scattering behaviour of natural and urban targets," IEE Proc. Radar, Sonar Navigat. (1994-2006), vol. 153, no. 3, p. 214,2006

[38] I. Joughin, B. E. Smith, I. M. Howat, D. Floricioiu, R. B. Alley, M. Truffer, and M. Fahnestock, "Seasonal to decadal scale variations in the surface velocity of Jakobshavn Isbrae, Greenland: Observation and model-based analysis," J. Geophys. Res., vol. 117, 2012.

[39] R. Schneider, K. Papathanassiou, I. Hajnsek, and A. Moreira, "Polarimetric and interferometric characterization of coherent scatterers in urban areas," IEEE Trans. Geosci. Remote Sensing, vol. 44, no. 4, pp. 971-984, 2006.

[40] H. Skriver, F. Mattia, G. Satalino, A. Balenzano, V. R. Pauwels, N. E. Verhoest, and M. Davidson, "Crop classification using short-revisit multitemporal SAR data," IEEE J. Select. Topics Appl. Earth Observ. Remote Sensing, vol. 4, no. 2, pp. 423-431, 2011.

[41] A. K. Fung, Microwave Scattering and Emission Models and Their Applications. Boston, MA: Artech House, 1994.

[42] H. C. van de Hulst, Light Scattering by Small Particles. New York: Dover, 1981

[43] S. Nghiem, S. Yueh, R. Kwok, and F. Li, "Symmetry properties in polarimetric remote sensing," Radio Sci., vol. 27, no. 5, pp. 693-711, 1992.

[44] J.-S. Lee, M. R. Grunes, and G. de Grandi, "Polarimetric SAR speckle filtering and its implication for classification," IEEE Trans. Geosci. Remote Sensing, vol. 37, no. 5, pp. 2363-2373, 1999.

[45] A. Freeman and S. L. Durden, "A three-component scattering model for polarimetric SAR data," IEEE Trans. Geosci. Remote Sensing, vol. 36, no. 3, pp. 963-973, 1998.

[46] Y. Yamaguchi, T. Moriyama, M. Ishido, and H. Yamada, "Fourcomponent scattering model for polarimetric SAR image decomposition," IEEE Trans. Geosci. Remote Sensing, vol. 43, no. 8, pp. 1699-1706, 2005.

[47] Y. Yamaguchi, Y. Yajima, and H. Yamada, "A four-component decomposition of POLSAR images based on the coherency matrix," IEEE Geosci. Remote Sensing Lett., vol. 3, no. 3, pp. 292-296, 2006.

[48] A. Freeman, "Fitting a two-component scattering model to polarimetric SAR data from forests," IEEE Trans. Geosci. Remote Sensing, vol. 45, no. 8, pp. 2583-2592, 2007.

[49] I. Hajnsek, T. Jagdhuber, H. Schon, and K. P. Papathanassiou, "Potential of estimating soil moisture under vegetation cover by means of PolSAR," IEEE Trans. Geosci. Remote Sensing, vol. 47, no. 2, pp. 442-454, 2009.

[50] T. Jagdhuber. (2012). Soil parameter retrieval under vegetation cover using SAR polarimetry. Ph.D. dissertation, Univ. Potsdam, Germany [Online]. Available: http//: opus.kobv.de

[51] J. M. Lopez-Sanchez, I. Hajnsek, and J. Ballester-Berman, "First demonstration of agriculture height retrieval with POLinSAR airborne data," IEEE Geosci. Remote Sensing Lett., vol. 9, no. 2, pp. 242-246, 2012

[52] R. Bamler and P. Hartl, "Synthetic aperture radar interferometry," Inverse Problems, vol. 14, no. 4, pp. R1-R54, Aug. 1998. 
[53] D. Massonnet and K. L. Feigl, "Radar interferometry and its application to changes in the Earth's surface," Rev. Geophys., vol. 36, no. 4, pp. 441-500, 1998.

[54] R. F. Hanssen, Radar Interferometry: Data Interpretation and Error Analysis. The Netherlands: Kluwer, 2001.

[55] L. C. Graham, "Synthetic interferometric radar for topographic mapping," Proc. IEEE, vol. 62, pp. 763-768, 1974.

[56] H. Zebker and R. M. Goldstein, "Topographic mapping from interferometric synthetic aperture radar observations," $J$. Geophys. Res., vol. 91, no. B5, pp. 4993-4999, 1986.

[57] R. Goldstein and H. Zebker, "Interferometric radar measurement of ocean surface currents," Nature, vol. 328, no. 20, pp. 707-709, 1987.

[58] A. L. Gray and P. J. Farris-Manning, "Repeat-pass interferometry with airborne synthetic aperture radar," IEEE Trans. Geosci. Remote Sensing, vol. 31, no. 1, pp. 180-191, Jan. 1993.

[59] R. E. Carande, "Estimating ocean coherence time using dualbaseline interferometric synthetic aperture radar," IEEE Trans. Geosci. Remote Sensing, vol. 32, no. 4, pp. 846-854, 1994.

[60] R. M. Goldstein, H. A. Zebker, and C. L. Werner, "Satellite radar interferometry: Two-dimensional phase unwrapping," Radio Sci., vol. 23, no. 4, pp. 713-720, July 1988.

[61] A. K. Gabriel, R. M. Goldstein, and H. A. Zebker, "Mapping small elevation changes over large areas: Differential radar interferometry," J. Geophys. Res., vol. 94, pp. 9183-9191, 1989.

[62] R. M. Goldstein, H. Engelhardt, B. Kamb, and R. M. Frolich, "Satellite radar interferometry for monitoring ice sheet motion: Application to an Antarctic ice stream," Science, vol. 262, pp. 763-768, 1993.

[63] M. A. Fahnestock, R. A. Bindschadler, R. Kwok, and K. C. Jezek, "Greenland ice sheet surface properties and ice flow from ERS-1 SAR imagery," Science, vol. 262, pp. 1530-1534, 1993.

[64] J. Askne and J. O. Hagberg, "Potential of interferometric SAR for classification of land surfaces," in Proc. IEEE Int. Geoscience and Remote Sensing Symp. (IGARSS), Tokyo, 1993.

[65] U. Wegmuller and C. L. Werner, "SAR interferometric signatures of forest," IEEE Trans. Geosci. Remote Sensing, vol. 33, no. 5, pp. 1153-1163, 1995.

[66] D. Massonnet, P. Briole, and A. Arnaud, "Deflation of mount Etna monitored by spaceborne radar interferometry," Nature, vol. 375, pp. 567-570, 1995.

[67] P. Dammert, M. Lepparanta, and J. Askne, "SAR interferometry over Baltic Sea ice," Int. J. Remote Sens., vol. 19, no. 16, pp. 3019-3037, 1998.

[68] S. R. Cloude and K. P. Papathanassiou, "Polarimetric SAR interferometry," IEEE Trans. Geosci. Remote Sensing, vol. 36, no. 5, pp. 1551-1565, Sept. 1998.

[69] A. Ferretti, C. Prati, and F. Rocca, "Nonlinear subsidence rate estimation using permanent scatterers in differential SAR interferometry," IEEE Trans. Geosci. Remote Sensing, vol. 38, no. 5, pp. 2202-2212, Sept. 2000.

[70] H. Breit, M. Eineder, J. Holzner, H. Runge, and R. Bamler, "Traffic monitoring using SRTM along-track interferometry," in Proc. IEEE Int. Geoscience and Remote Sensing Symp. (IGARSS), Toulouse, France, 2003.

[71] D. C. Ghiglia and M. D. Pritt, Two-Dimensional Phase Unwrapping: Theory, Algorithms, and Software. New York: Wiley, 1998.

[72] C. E. Livingstone, I. Sikaneta, C. H. Gierull, S. Chiu, A. Beaudoin, J. Campbell, J. Beaudoin, S. Gong, and T. A. Knight, "An airborne synthetic aperture radar (SAR) experiment to support RADARSAT-2 ground moving target inidication (GMTI)," Can. J. Remote Sens., vol. 28, no. 6, pp. 794-813, 2002.

[73] R. Romeiser, H. Breit, M. Eineder, H. Runge, P. Flament, J. de Karin, and J. Vogelzang, "Current measurements by SAR along-track interferometry from a space shuttle," IEEE Trans. Geosci. Remote Sensing, vol. 43, no. 10, pp. $2315-$ 2324, 1995.
[74] J. Hagberg, L. Ulander, and J. Askne, "Repeat-pass SAR interferometry over forested terrain," IEEE Trans. Geosci. Remote Sensing, vol. 33, no. 2, pp. 331-340, 1995.

[75] U. Wegmuller and C. Werner, "Retrieval of vegetation parameters with SAR interferometry," IEEE Trans. Geosci. Remote Sensing, vol. 35, no. 1, pp. 18-24, 1997.

[76] G. Krieger, A. Moreira, H. Fiedler, I. Hajnsek, M. Werner, M. Younis, and M. Zink, "TanDEM-X: A satellite formation for high-resolution SAR interferometry," IEEE Trans. Geosci. Remote Sensing, vol. 45, no. 11, pp. 3317-3341, 2007.

[77] H. A. Zebker and J. Villasenor, "Decorrelation in interferometric radar echoes," IEEE Trans. Geosci. Remote Sensing, vol. 30, no. 5, pp. 950-959, 1992.

[78] E. Rodriguez and J. Martin, "Theory and design of interferometric synthetic aperture radars," Radar and Signal Processing, IEE Proc. F (1988-1993), vol. 139, no. 2, pp. 147-159, 1992.

[79] F. Gatelli, A. M. Guamieri, F. Parizzi, P. Pasquali, C. Prati, and F. Rocca, "The wavenumber shift in SAR interferometry," IEEE Trans. Geosci. Remote Sensing, vol. 32, no. 4, pp. 855-865, July 1994.

[80] A. Ferretti, A. Monti Guarnieri, C. Prati, and F. Rocca, "Multi-baseline interferometric techniques and applications," in Proc. Fringe Workshop, Zurich, 1996.

[81] M. Eineder and N. Adam, "A maximum-likelihood estimator to simultaneously unwrap, geocode, and fuse SAR interferograms from different viewing geometries into one digital elevation model," IEEE Trans. Geosci. Remote Sensing, vol. 43, no. 1, pp. 24-36, 2005.

[82] M. Lachaise, U. Balss, T. Fritz, and H. Breit, "The dual-baseline interferometric processing chain for the TanDEM-X mission," in Proc. IEEE Int. Geoscience and Remote Sensing Symp. (IGARSS), Munich, 2012.

[83] G. Krieger, I. Hajnsek, K. Papathanassiou, M. Younis, and A. Moreira, "Interferometric synthetic aperture radar (SAR) missions employing formation flying," Proc. IEEE, vol. 98, no. 5, pp. 816-843, 2010.

[84] T. Fritz, C. Rossi, N. Yague-Martinez, F. Rodriguez-Gonzalez, M. Lachaise, and H. Breit, "Interferometric processing of TanDEM-X data," in Proc. IEEE Int. Geoscience and Remote Sensing Symp. (IGARSS), Vancouver, Canada, 2011.

[85] P. Prats, M. Rodriguez-Cassola, L. Marotti, M. Nannini, S. Wollstadt, D. Schulze, N. Tous-Ramon, M. Younis, G. Krieger, and A. Reigber, "TAXI: A versatile processing chain for experimental TanDEM-X product evaluation," in Proc. IEEE Int. Geoscience and Remote Sensing Symp. (IGARSS), Honolulu, Hawaii, 2010.

[86] F. Rocca, "Modeling interferogram stacks," IEEE Trans. Geosci. Remote Sensing, vol. 45, no. 10, pp. 3289-3299, 2007.

[87] Z.-W. Xu, J. Wu, and Z.-S. Wu, "A survey of ionospheric effects on space-based radar," Waves Random Media, vol. 14, pp. S189S273, 2004.

[88] D. P. Belcher, "Theoretical limits on SAR imposed by the ionosphere," IET Radar, Sonar Navigat., vol. 2, no. 6, 2008.

[89] J. van Zyl, "The Shuttle Radar Topography Mission (SRTM): A breakthrough in remote sensing of topography," Acta Astronaut., vol. 48, no. 5, pp. 559-565, 2001.

[90] A. Ortega-Guerrero, D. Rudolph, and J. Cherry, "Analysis of long-term land subsidence near Mexico City: Field investigations and predictive modeling," Water Resour. Res., vol. 35, no. 11, pp. 3327-3341, 1999.

[91] P. Berardino, G. Fornaro, R. Lanari, and E. Sansosti, "A new algorithm for surface deformation monitoring based on small baseline differential SAR interferograms," IEEE Trans. Geosci. Remote Sensing, vol. 40, no. 11, pp. 2375-2383, Nov. 2002.

[92] O. Mora, J. J. Mallorqui, and A. Broquetas, "Linear and nonlinear terrain deformation maps from a reduced set of interferometric SAR images," IEEE Trans. Geosci. Remote Sensing, vol. 41, no. 10, pp. 2243-2253, Oct. 2003. 
[93] R. Lanari, O. Mora, M. Manunta, J. J. Mallorqui, P. Berardino, and E. Sansosti, "A small-baseline approach for investigating deformations on full-resolution differential SAR interferograms," IEEE Trans. Geosci. Remote Sensing, vol. 42, no. 7, pp. 1377-1386, July 2004.

[94] A. Ferretti, A. Fumagalli, F. Novali, C. Prati, F. Rocca, and A. Rucci, "A new algorithm for processing interferometric datastacks: SqueeSAR," IEEE Trans. Geosci. Remote Sensing, vol. 49, no. 9, pp. 3460-3470, 2011.

[95] C. Colesanti, A. Ferretti, F. Novali, C. Prati, and F. Rocca, "SAR monitoring of progressive and seasonal ground deformation using the permanent scatterers technique," IEEE Trans. Geosci. Remote Sensing, vol. 41, no. 7, pp. 1685-1701, 2003.

[96] A. Hooper, H. Zebker, P. Segall, and B. Kampes, "A new method for measuring deformation on volcanoes and other natural terrains using InSAR persistent scatterers," Geophys. Res. Lett., vol. 31, no. 23, 2004.

[97] M. Costantini, S. Falco, F. Malvarosa, and F. Minati, "A new method for identification and analysis of persistent scatterers in series of SAR images," in Proc. IEEE Int. Geoscience and Remote Sensing Symp. (IGARSS), 2008.

[98] A. Monti Guarnieri and S. Tebaldini, "On the exploitation of target statistics for SAR interferometry applications," IEEE Trans. Geosci. Remote Sensing, vol. 46, no. 11, pp. 3436-3443, 2008.

[99] R. Iglesias, D. Monells, G. Centolanza, J. Mallorquí, and P. López-Dekker, "A new approach to DInSAR pixel selection with spectral correlation along time between sublooks," in Proc. European Conf. Synthetic Aperture Radar, Nuremberg, Germany, 2012, pp. 665-668.

[100] M. Sanjuan-Ferrer, I. Hajnsek, and K. Papathanassiou, "Analysis of the detection performance of coherent scatterers in SAR images," in Proc. IEEE Int. Geoscience and Remote Sensing Symp. (IGARSS), 2012.

[101] M. Costantini, F. Malvarosa, F. Minati, L. Pietranera, and G. Milillo, "A three-dimensional phase unwrapping algorithm for processing of multitemporal SAR interferometric measurements," in Proc. IEEE Int. Geoscience and Remote Sensing Symp. (IGARSS), 2002, vol. 3, pp. 1741-1743.

[102] A. Hooper and H. Zebker, "Phase unwrapping in three dimensions with application to InSAR time series," J. Opt. Soc. Amer. A, Opt. Image Sci., vol. 24, no. 9, pp. 2737-2747, 2007.

[103] F. van Leijen, R. Hanssen, P. Marinkovic, and B. Kampes, "Spatio-temporal phase unwrapping using integer leastsquares," in Proc. Fringe Workshop, 2006, vol. 610, p. 62.

[104] D. Perissin and F. Rocca, "High-accuracy urban DEM using permanent scatterers," IEEE Trans. Geosci. Remote Sensing, vol. 44, no. 11, pp. 3338-3347, 2006

[105] O. Mora, R. Arbiol, V. Pala, A. Adell, and M. Torre, "Generation of accurate DEMs using DInSAR methodology (TopoDInSAR)," IEEE Geosci. Remote Sensing Lett., vol. 3, no. 4, pp. 551-554, 2006.

[106] Y. Wang, X. Zhu, Y. Shi, and R. Bamler, "Operational TomoSAR processing using TerraSAR-X high resolution spotlight stacks from multiple view angles," in Proc. IEEE Int. Geoscience and Remote Sensing Symp. (IGARSS), 2012, pp. 7047-7050.

[107] S. R. Cloude and K. P. Papathanassiou, "Polarimetric SAR interferometry," IEEE Trans. Geosci. Remote Sensing, vol. 36, no. 5, pp. 1551-1565, 1998.

[108] G. Krieger, K. P. Papathanassiou, and S. R. Cloude, "Spaceborne polarimetric SAR interferometry: Performance analysis and mission concepts," EURASIP J. Appl. Signal Processing, vol. 2005, pp. 3272-3292, 2005.

[109] S. R. Cloude and K. P. Papathanassiou, "Three-stage inversion process for polarimetric SAR interferomtry," IEE Proc. Radar, Sonar Navigat. (1994-2006), vol. 150, no. 3, pp. 125-134, 2003.

[110] R. N. Treuhaft and P. R. Siqueira, "The vertical structure of vegetated land surfaces from interferometric and polarimetric radar," Radio Sci., vol. 35, no. 1, pp. 141-177, 2000.
[111] R. N. Treuhaft, S. N. Madsen, M. Moghaddam, and J. J. van Zyl, "Vegetation characteristics and underlying topography from interferometric data," Radio Sci., vol. 31, pp. 1449-1495, 1996.

[112] F. Garestier, P. C. Dubois-Fernandez, and I. Champion, "Forest height inversion using high-resolution P-band Pol-InSAR data," IEEE Trans. Geosci. Remote Sensing, vol. 46, no. 11, pp. 3544-3559, 2008.

[113] F. Garestier and T. Le Toan, "Estimation of the backscatter vertical profile of a pine forest using single baseline P-band (Pol-) InSAR data," IEEE Trans. Geosci. Remote Sensing, vol. 48, no. 9, pp. 3340-3348, 2010.

[114] F. Garestier and T. Le Toan, "Forest modeling for height inversion using single-baseline InSAR/Pol-InSAR data," IEEE Trans. Geosci. Remote Sensing, vol. 48, no. 3, pp. 1528-1539, 2010.

[115] R. N. Treuhaft and S. R. Cloude, "The structure of oriented vegetation from polarimetric interferometry," IEEE Trans. Geosci. Remote Sensing, vol. 37, no. 5, pp. 2620-2624, 1999.

[116] I. Hajnsek and S. R. Cloude, "Differential extinction estimation over agricultural vegetation from POLinSAR," in Proc. 2nd Int. Workshop POLinSAR, Frascati, Italy, 2005.

[117] J. D. Ballester-Berman, J. M. Lopez-Sanchez, and J. FortunyGuasch, "Retrieval of biophysical parameters of agricultural crops using polarimetric SAR interferometry," IEEE Trans. Geosci. Remote Sensing, vol. 43, no. 4, pp. 683-694, 2005.

[118] J. M. Lopez-Sanchez, J. D. Ballester-Berman, and J. FortunyGuasch, "Indoor wide-band polarimetric measurements on maize plants: A study of the differential extinction coefficient," IEEE Trans. Geosci. Remote Sensing, vol. 44, no. 4, pp. 758-767, 2006.

[119] S. R. Cloude, "Polarization coherence tomography," Radio Sci., vol. 41, no. 4, p. RS4017, 2006.

[120] S. R. Cloude, "Dual-baseline coherence tomography," IEEE Geosci. Remote Sensing Lett., vol. 4, no. 1, pp. 127-131, 2007.

[121] F. Kugler, F. N. Koudogbo, K. Gutjahr, and K. P. Papathanassiou, "Frequency effects in Pol-InSAR forest height estimation," in Proc. 2006, Gth European Conf. Synthetic Aperture Radar (EUSAR).

[122] F. Kugler, S.-K. Lee, and K. Papathanassiou, "Estimation of forest vertical sructure parameter by means of multi-baseline Pol-InSAR," in Proc. IEEE Int. Geoscience and Remote Sensing Symp. (IGARSS), Cape Town, South Africa, 2009.

[123] K. Papathanassiou and S. R. Cloude, "The effect of temporal decorrelation on the inversion of forest parameters from PoI-InSAR data," in Proc. IEEE Int. Geoscience and Remote Sensing Symp. (IGARSS), Toulouse, France, 2003.

[124] S. Lee, F. Kugler, K. Papathanassiou, and I. Hajnsek, "Temporal decorrelation of forest vegetation at L- and P-band," in Proc. 2006, 7th European Conference on Synthetic Aperture Radar (EUSAR), Friedrichshafen, Germany, 2008.

[125] S.-K. Lee, F. Kugler, K. Papathanassiou, and I. Hajnsek, "Quantification of temporal decorrelation effects at L-band for polarimetric SAR interferometry applications," IEEE J. Select. Topics Appl. Earth Observ. Remote Sensing (JSTARS), to be published.

[126] S.-K. Lee, F. Kugler, I. Hajnsek, and K. P. Papathanassiou, "The impact of temporal decorrelation over forest terrain in polarimetric SAR interferometry," in Proc. 2nd Int. Workshop POLinSAR, Frascati, Italy, 2009.

[127] M. Lavalle, M. Simard, and S. Hensley, "A temporal decorrelation model for polarimetric radar interferometers," IEEE Trans. Geosci. Remote Sensing, vol. 50, no. 7, pp. 2880-2888, 2012.

[128] J. Praks, F. Kugler, K. Papathanassiou, I. Hajnsek, and M. Hallikainen, "Tree height estimation for boreal forest by means of Land X-band POLinSAR and HUTSCAT profiling scatterometer," IEEE Geosci. Remote Sensing Lett., vol. 4, no. 3, pp. 466-470, 2007.

[129] F. Garestier, P. C. Dubois-Fernandez, and K. P. Papathanassiou, "Pine forest height inversion using single-pass X-band POLinSAR data," IEEE Trans. Geosci. Remote Sensing, vol. 46, no. 1, pp. 59-68, 2008. 
[130] F. Garestier, P. C. Dubois-Fernandez, D. Guyon, and T. Le Toan, "Forest biophysical parameter estimation using L-band P-band polarimetric SAR data," IEEE Trans. Geosci. Remote Sensing, vol. 47, no. 10, pp. 3379-3388, 2009.

[131] I. Hajnsek, F. Kugler, S.-K. Lee, and K. P. Papathanassiou, "Tropical-forest-parameter estimation by means of Pol-InSAR: The INDREX-II campaign," IEEE Trans. Geosci. Remote Sensing, vol. 47, no. 2, pp. 481-493, 2009.

[132] S.-K. Lee, F. Kugler, I. Hajnsek, and K. Papathanassiou, "Multibaseline Pol-InSAR forest height estimation in the presence of temporal decorrelation," in Proc. 2010, 8th European Conf. Synthetic Aperture Radar (EUSAR), Aachen, Germany.

[133] S. R. Cloude, K. P. Papathanassiou, and W. M. Boerner, "A fast method for vegetation correction in topographic mapping using polarimetric radar interferometry," in Proc. 2010, 3rd European Conf. Synthetic Aperture Radar (EUSAR), Munich, 2000.

[134] L. Ferro-Famil, M. Neumann, and Y. Huang, "Multi-baseline POL-inSAR statistical techniques for the characterization of distributed media," in Proc. IEEE Int. Geoscience and Remote Sensing Symp. (IGARSS), Cape Town, South Africa, 2009.

[135] C. López-Martínez and K. P. Papathanassiou, "Cancellation of scattering mechanisms in POLinSAR: Application to underlying topography estimation," IEEE Trans. Geosci. Remote Sensing, to be published.

[136] T. Mette, K. Papathanassiou, and I. Hajnsek, "Estimating forest biomass from polarimetric interferometric SAR data in combination with forest allometry - results from temperate spruce forest test site Traunstein," in Proc. Retrieval Bio and Geophysical Parameters from SAR Data for Land Applications, Innsbruck, Austria, 2004.

[137] T. Mette, K. Papathanassiou, and I. Hajnsek, "Biomass estimation from polarimetric SAR interferometry over heterogeneous forest terrain," in Proc. IEEE Int. Geoscience and Remote Sensing Symp. (IGARSS), Anchorage, AK, 2004.

[138] S. R. Cloude, M. Brolly, and I. H. Woodhouse, "A study of forest vertical structure estimation using coherence tomography coupled to a macro-ecological scattering model," in Proc. IEEE Int. Geoscience and Remote Sensing Symp. (IGARSS), Cape Town, South-Africa, 2009.

[139] R. N. Treuhaft, F. G. Gonçalves, J. B. Drake, B. D. Chapman, J. R. dos Santos, L. V. Dutra, P. Graça, and G. H. Purcell, "Biomass estimation in a tropical wet forest using Fourier transforms of profiles from lidar or interferometric SAR," Geophys. Res. Lett., vol. 37, no. 23, 2010.

[140] J. Dall, "InSAR elevation bias caused by penetration into uniform volumes," IEEE Trans. Geosci. Remote Sensing, vol. 45, no. 7, pp. 2319-2324, 2007.

[141] J. J. Sharma, I. Hajnsek, and K. P. Papathanassiou, "Multifrequency Pol-InSAR signatures of a subpolar glacier," in Proc. 3rd Int. Workshop POLinSAR, Frascati, Italy, 2007.

[142] S. Oveisgharan and H. A. Zebker, "Estimating snow accumulation from InSAR correlation observations," IEEE Trans. Geosci. Remote Sensing, vol. 45, no. 1, pp. 10-20, 2007.

[143] E. Weber Hoen and H. A. Zebker, "Penetration depths inferred from interferometric volume decorrelation observed over the Greenland ice sheet," IEEE Trans. Geosci. Remote Sensing, vol. 38, no. 6, pp. 2571-2583, 2000.

[144] J. J. Sharma, I. Hajnsek, and K. P. Papathanassiou, "Vertical profile reconstruction with Pol-InSAR data of a subpolar glacier," in Proc. IEEE Int. Geoscience and Remote Sensing Symp. (IGARSS), Barcelona, Spain, 2007.

[145] J. J. Sharma, I. Hajnsek, and K. P. Papathanassiou, "Longwavelength POLinSAR for glacier ice extinction estimation," in Proc. 2010, 8th European Conf. Synthetic Aperture Radar (EU$S A R)$, Aachen, Germany.
[146] J. Sharma, I. Hajnsek, K. Papathanassiou, and A. Moreira, "Estimation of glacier ice extinction using long-wavelength airborne POLinSAR," IEEE Trans. Geosci. Remote Sensing, to be published.

[147] J. Homer, I. Longstaff, and G. Callaghan, "High resolution 3-D SAR via multi-baseline interferometry," in Proc. IEEE Int. Geoscience and Remote Sensing Symp. (IGARSS), Lincoln, NE, 1996.

[148] S. Tebaldini, "Single and multipolarimetric SAR tomography of forested areas: A parametric approach," IEEE Trans. Geosci. Remote Sensing, vol. 48, no. 5, pp. 2375-2387, 2010.

[149] M. Nannini, R. Scheiber, R. Horn, and A. Moreira, "First 3D reconstructions of targets hidden beneath foliage by means of polarimetric SAR tomography," IEEE Geosci. Remote Sensing Lett., vol. 9, no. 1, pp. 60-64, 2012.

[150] E. Candès and M. Wakin, "An introduction to compressive sampling," IEEE Signal Processing Mag., vol. 25, no. 2, pp. 21-30, 2008.

[151] F. Lombardini, "Differential tomography: A new framework for SAR interferometry," IEEE Trans. Geosci. Remote Sensing, vol. 43, no. 1, pp. 37-44, 2005.

[152] G. Fornaro, D. Reale, and F. Serafino, "Four-dimensional SAR imaging for height estimation and monitoring of single and double scatterers," IEEE Trans. Geosci. Remote Sensing, vol. 47, no. 1, pp. 224-237, 2009.

[153] X. Zhu and R. Bamler, "Let's do the time warp: Multicomponent nonlinear motion estimation in differential SAR tomography," IEEE Geosci. Remote Sensing Lett., vol. 8, no. 4, pp. 735739, 2011.

[154] X. Zhu and R. Bamler, "Very high resolution spaceborne SAR tomography in urban environment," IEEE Trans. Geosci. Remote Sensing, vol. 48, no. 12, pp. 4296-4308, 2010.

[155] G. Fornaro, F. Serafino, and D. Reale, "4-D SAR imaging: The case study of Rome," IEEE Geosci. Remote Sensing Lett., vol. 7, no. 2, pp. 236-240, 2010.

[156] D. Reale, G. Fornaro, A. Pauciullo, X. Zhu, and R. Bamler, "Tomographic imaging and monitoring of buildings with very high resolution SAR data," IEEE Geosci. Remote Sensing Lett., vol. 8, no. 4, pp. 661-665, 2011.

[157] X. Zhu and R. Bamler, "Demonstration of super-resolution for tomographic SAR imaging in urban environment," IEEE Trans. Geosci. Remote Sensing, vol. 50, no. 8, pp. 3150-3157, 2012.

[158] E. Aguilera, M. Nannini, and A. Reigber, "Wavelet-based compressed sensing for SAR tomography of forested areas," IEEE Trans. Geosci. Remote Sensing, to be published.

[159] M. Soumekh, "Reconnaissance with slant plane circular SAR imaging," IEEE Trans. Image Processing," vol. 5, no. 8, pp. 1252-1265, 1996.

[160] A. Ishimaru, T. Chan, and Y. Kuga, "An imaging technique using confocal circular synthetic aperture radar," IEEE Trans. Geosci. Remote Sensing, vol. 36, no. 5, pp. 1524-1530, 1998.

[161] H. Cantalloube, E. Colin-Koeniguer, and H. Oriot, "High resolution SAR imaging along circular trajectories," in Proc. IEEE Int. Geoscience and Remote Sensing Symp. (IGARSS), 2007, pp. 850-853.

[162] E. Ertin, C. Austin, S. Sharma, R. Moses, and L. Potter, "Gotcha experience report: Three-dimensional SAR imaging with complete circular apertures," in Proc. SPIE, 2007, vol. 6568, p. 656802.

[163] H. Oriot and H. Cantalloube, "Circular SAR imagery for urban remote sensing," in Proc. 7th Eurpean Conf. Synthetic Aperture Radar (EUSAR), 2008, pp. 1-4.

[164] M. Pinheiro, P. Prats, R. Scheiber, M. Nannini, and A. Reigber, "Tomographic 3D reconstruction from airborne circular SAR," in Proc. IEEE Int. Geoscience and Remote Sensing Symp. (IGARSS), 2009, vol. 3, pp. III-21.

[165] O. Ponce, P. Prats, R. Scheiber, A. Reigber, and A. Moreira, "Multibaseline 3-D circular SAR imaging at L-band," in Proc. 9th European Conf. Synthetic Aperture Radar (EUSAR), 2012, pp. 113-116. 
[166] O. Ponce, P. Prats, R. Scheiber, A. Reigber, and A. Moreira, "Polarimetric 3-D reconstruction from multicircular SAR at P-band," in Proc. IEEE Int. Geoscience and Remote Sensing Symp. (IGARSS), 2012, pp. 3130-3133.

[167] A. Moreira, I. Hajnsek, G. Krieger, K. Papathanassiou, M. Eineder, F. De Zan, M. Younis, and M. Werner, "Tandem-L: Monitoring the Earth's dynamics with InSAR and POLinSAR," in Proc. Int. Workshop Applications Polarimetry and Polarimetric Interferometry (POLinSAR), Frascati, Italy, Jan. 2009.

[168] J. H. Blythe, "Radar systems," U.S. Patent 4253 098, Feb., 1981.

[169] A. Currie and M. Brown, "Wide-swath SAR," Radar and Signal Processing, IEE Proc. F (1988-1993), vol. 139, no. 2, pp. 122-135, 1992.

[170] J. T. Kare, "Moving receive beam method and apparatus for synthetic aperture radar," U.S. Patent 6175 326, Jan. 2001.

[171] G. D. Callaghan and I. Longstaff, "Wide-swath space-borne SAR using a quad-element array," IEE Proc. Radar, Sonar Navigat. (1994-2006), vol. 146, no. 3, pp. 155-165, 1999.

[172] M. Süß, B. Grafmüller, and R. Zahn, "A novel high resolution, wide swath SAR," in Proc. IEEE Int. Geoscience and Remote Sensing Symp. IGARSS'01, vol. 3, pp. 1013-1015.

[173] G. Krieger, J. Mittermayer, S. Buckreuss, M. Wendler, T. Sutor, and A. Moreira, "Sector imaging radar for enhanced vision," Aerosp. Sci. Technol., no. 7, pp. 147-158, 2003.

[174] M. Younis, C. Fischer, and W. Wiesbeck, "Digital beamforming in SAR systems," IEEE Trans. Geosci. Remote Sensing, vol. 41, no. 7, pp. 1735-1739, July 2003.

[175] G. Krieger and A. Moreira, "Potential of digital beamforming in bi-and multistatic SAR," in Proc. IEEE Int. Geoscience and Remote Sensing Symp. (IGARSS), 2003, vol. 1, pp. 527-529.

[176] G. Krieger, N. Gebert, and A. Moreira, "Unambiguous SAR signal reconstruction from non-uniform displaced phase centre sampling," IEEE Geosci. Remote Sensing Lett., vol. 1, no. 4, pp. 260-264, Oct. 2004.

[177] J. Klare, A. Brenner, and J. Ender, "A new airborne radar for 3D imaging-Image formation using the ARTINO principle," in Proc. European Conf. Synthetic Aperture Radar, Dresden, Germany, 2006.

[178] G. Krieger, N. Gebert, and A. Moreira, "Multidimensional waveform encoding: A new digital beamforming technique for synthetic aperture radar remote sensing," IEEE Trans. Geosci. Remote Sensing, vol. 46, no. 1, pp. 31-46, Jan. 2008

[179] G. Krieger, N. Gebert, M. Younis, F. Bordoni, A. Patyuchenko, and A. Moreira, "Advanced concepts for ultra-wide swath SAR imaging," in Proc. European Conf. Synthetic Aperture Radar, Friedrichshafen, Germany, June 2008.

[180] J. Li and P. Stoica, MIMO Radar Signal Processing. Hoboken, NJ: Wiley, 2009.

[181] J. Ender and J. Klare, "System architectures and algorithms for radar imaging by MIMO-SAR," in Proc. IEEE Radar Conf., 2009, pp. 1-6.

[182] N. Gebert, G. Krieger, and A. Moreira, "Digital beamforming on receive: Techniques and optimization strategies for highresolution wide-swath SAR imaging," IEEE Trans. Aerosp. Electron. Syst., vol. 45, pp. 564-592, 2009.

[183] F. Bordoni, M. Younis, E. Varona, N. Gebert, and G. Krieger, "Performance investigation on scan-on-receive and adaptive digital beam-forming for high-resolution wide-swath synthetic aperture radar," in Proc. Int. ITG Workshop Smart Antennas, 2009.
[184] M. Younis, S. Huber, A. Patyuchenko, F. Bordoni, and G. Krieger. (2009, June). Performance comparison of reflector- and planar-antenna based digital beam-forming SAR. Int. J. Antennas Propagat.[Online]. 2009. Available: http://www.hindawi.com/ journals/ijap/2009

[185] A. Freeman, G. Krieger, P. Rosen, M. Younis, W. Johnson, S. Huber, R. Jordan, and A. Moreira, "SweepSAR: Beam-forming on receive using a reflector-phased array feed combination for spaceborne SAR," in Proc. IEEE Radar Conf. (RadarCon'09), Pasadena, CA, May 2009.

[186] N. Gebert, G. Krieger, and A. Moreira, "Multichannel azimuth processing in ScanSAR and TOPS mode operation," IEEE Trans. Geosci. Remote Sensing, vol. 48, no. 7, pp. 2994-3008, 2010.

[187] C. Fischer, C. Heer, and R. Werninghaus, "Development of a high-resolution wide-swath SAR demonstrator," in Proc. European Conf. Synthetic Aperture Radar (EUSAR), Aachen, Germany, 2010.

[188] C. Schaefer, C. Heer, and M. Ludwig, "Advanced C-band instrument based on digital beamforming," in Proc. European Conf. Synthetic Aperture Radar (EUSAR), Aachen, Germany, 2010.

[189] D. Cristallini, D. Pastina, and P. Lombardo, "Exploiting MIMO SAR potentialities with efficient cross-track constellation configurations for improved range resolution," IEEE Trans. Geosci. Remote Sensing, vol. 49, no. 1, pp. 38-52, 2011.

[190] W. Wang, "Space-time coding MIMO-OFDM SAR high-resolution imaging," IEEE Trans. Geosci. Remote Sensing, vol. 49, no. 8, pp. 3094-3104, 2011.

[191] N. Gebert, F. de Almeida, and G. Krieger, "Airborne demonstration of multichannel SAR imaging," IEEE Geosci. Remote Sensing Lett., vol. 8, no. 5, pp. 963-967, 2011.

[192] S. Huber, M. Younis, A. Patyuchenko, G. Krieger, and A. Moreira, "Spaceborne reflector SAR systems with digital beamforming," IEEE Trans. Aerosp. Electron. Syst., vol. 48, no. 4, pp. 3473-3493, 2012.

[193] M. Villano, G. Krieger, and A. Moreira, "Staggered-SAR for highresolution wide-swath imaging," in Proc. IET Radar, Glasgow, U.K., 2012.

[194] W. Wang, "Mitigating range ambiguities in high-PRF SAR with OFDM waveform diversity," IEEE Geosci. Remote Sensing Lett., vol. 10, no. 1, pp. 101-105, 2013.

[195] G. Krieger, "MIMO-SAR: Opportunities and pitfalls," IEEE Trans. Geosci. Remote Sensing, to be published.

[196] J. Kim, M. Younis, P. Prats-Iraola, M. Gabele, and G. Krieger, "First spaceborne demonstration of digital beamforming for azimuth ambiguity suppression," IEEE Trans. Geosci. Remote Sensing, vol. 51, no. 1, pp. 579-590, Jan. 2013.

[197] P. Prats-Iraola, R. Scheiber, L. Marotti, S. Wollstadt, and A. Reigber, "TOPS interferometry with TerraSAR-X," IEEE Trans. Geosci. Remote Sensing, vol. 50, no. 8, pp. 3179-3188, 2012.

[198] J. Kim, "Multiple-input multiple-output synthetic aperture radar for multimodal operation," Ph.D. dissertation, Karlsruhe Inst. Technol. (KIT), Germany, 2012.

[199] J. Kim, M. Younis, A. Moreira, and W. Wiesbeck, "A novel OFDM chirp waveform scheme for use of multiple transmitters in SAR," IEEE Geosci. Remote Sensing Lett., vol. 10, no. 3, pp. 568-572, May 2013. 